University of Louisville

ThinkIR: The University of Louisville's Institutional Repository

8-2013

\title{
The characterization of wing-wing vortex interactions of a tandem flapping wing configuration and its relationship to the phase angle and wing spacing.
}

Timothy Michael Broering 1985-

University of Louisville

Follow this and additional works at: https://ir.library.louisville.edu/etd

\section{Recommended Citation}

Broering, Timothy Michael 1985-, "The characterization of wing-wing vortex interactions of a tandem flapping wing configuration and its relationship to the phase angle and wing spacing." (2013). Electronic Theses and Dissertations. Paper 157.

https://doi.org/10.18297/etd/157

This Doctoral Dissertation is brought to you for free and open access by ThinkIR: The University of Louisville's Institutional Repository. It has been accepted for inclusion in Electronic Theses and Dissertations by an authorized administrator of ThinkIR: The University of Louisville's Institutional Repository. This title appears here courtesy of the author, who has retained all other copyrights. For more information, please contact thinkir@louisville.edu. 
THE CHARACTERIZATION OF WING-WING VORTEX INTERACTIONS OF A TANDEM FLAPPING WING CONFIGURATION AND ITS RELATIONSHIP TO THE PHASE ANGLE AND WING SPACING

\title{
By
}

Timothy Michael Broering, Jr.

B.S. University of Louisville, 2008

M.Eng. University of Louisville, 2009

\author{
A Dissertation \\ Submitted to the Faculty of the \\ Speed School of Engineering of the University of Louisville \\ in Partial Fulfillment of the Requirements \\ for the Degree of
}

Doctor of Philosophy

Department of Mechanical Engineering

University of Louisville

Louisville, Kentucky

August 2013 

THE CHARACTERIZATION OF WING-WING VORTEX INTERACTIONS OF A TANDEM FLAPPING WING CONFIGURATION AND ITS RELATIONSHIP TO THE PHASE ANGLE AND WING SPACING

$$
\text { By }
$$

Timothy Michael Broering, Jr.

B.S. University of Louisville, 2008

M.Eng. University of Louisville, 2009

A Dissertation Approved on

July 17, 2013

by the following Dissertation Committee:

Dr. Yongsheng Lian: Dissertation Director

Dr. Stuart Williams

Dr. Roger Bradshaw

Dr. Timothy Dowling 


\section{ACKNOWLEDGMENTS}

I would not be writing this today without the support and guidance from a great many people to whom I owe a great deal. First, I want to thank my advisor and mentor Dr. Yongsheng Lian, for all of the time and effort he had provided to me. I am also grateful for the number of opportunities he provided me with outside of our CFD lab, from research to teaching. None of this would have been possible without his direction and expertise, and the kindness and patience he has shown me these past four years are more than I deserve. I hope that I can live up to the example he has set for me.

I'd also like to thank my fellow grad students with whom I have had the pleasure of working with these past few years. They made my time in the lab far more enjoyable than it would have been otherwise.

I would also like to thank my committee members for volunteering to take time out of their busy schedules to review my work. Their time and effort is greatly appreciated.

And finally, I'd like to thank my family, especially my parents, for their love and support. Their words of encouragement kept me going, especially this past year, and I don't think I would have made it without their help. They never stopped believing in me, even when I didn't believe in myself. 


\section{ABSTRACT \\ THE CHARACTERIZATION OF WING-WING VORTEX INTERACTIONS OF A TANDEM FLAPPING WING CONFIGURATION AND ITS RELATIONSHIP TO THE PHASE ANGLE AND WING SPACING}

Timothy Michael Broering, Jr.

July 17,2013

A number of flying insects make use of tandem wing configurations, suggesting that such a setup may have potential advantages over a single wing at low Reynolds numbers. Dragonflies, which are fast and highly maneuverable, demonstrate well, the potential performance of such a configuration. In a tandem wing configuration, the hindwing often operates in the wake of the forewing and, hence, its performance is affected by the vortices shed by the forewing. Changes in the phase angle between the flapping motions of the fore and hind wings, as well as the spacing between them, can affect the resulting vortex/wing and vortex/vortex interactions.

In this thesis flapping wings in a tandem configuration were simulated using an incompressible Navier-Stokes solver on composite overlapping grids. Harmonic single frequency sinusoidal oscillations consisting of combined pitch and plunge motions were used for the flapping wing kinematics at a Strouhal number of 0.3. Different wing spacings ranging from 0.1 chords to 1 chord were tested at three different phase angles, $0^{\circ}, 90^{\circ}$ and $180^{\circ}$. It was found that changes in the spacing and phase angle affected the timing of the interaction between the vortex shed from the forewing and the hindwing. 
Such an interaction affects the LEV formation on the hindwing and results in changes to the aerodynamic force production and efficiencies of the hindwing.

It is also observed that changing the phase angle has a similar effect as changing the spacing. The results further show that at different spacings the peak force generation occurs at different phase angles, as do the peak efficiencies. The aerodynamics of the hindwing was also compared in detail to a single wing, with the same geometry and undergoing the same flapping kinematics, to determine the effect of vortex shedding from the forewing on the hindwing, as well as how the phase angle affects the interaction. The average lift, thrust and power coefficients and the average efficiency of the fore and hind wings were compared to a single wing to determine how the tandem wing interaction affects performance. 


\section{TABLE OF CONTENTS}

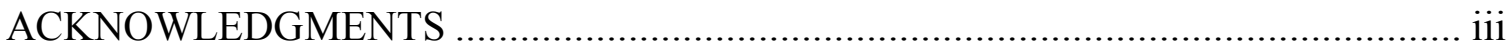

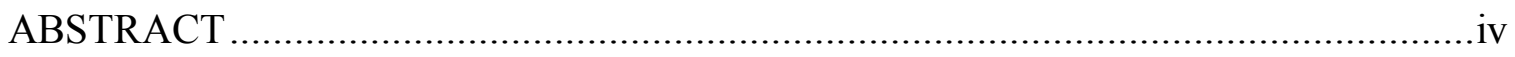

LIST OF FIGURES ……................................................................................... ix

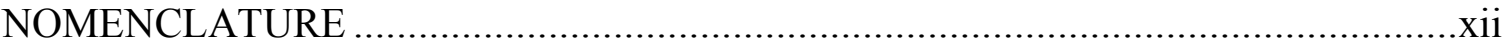

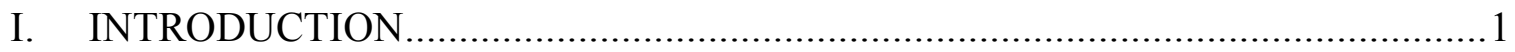

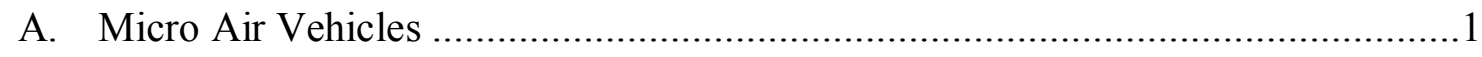

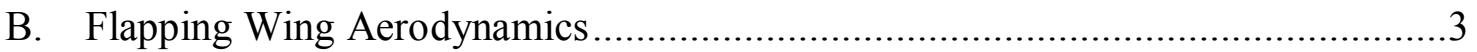

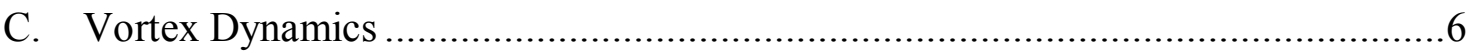

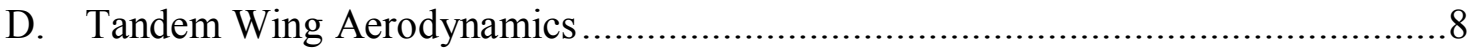

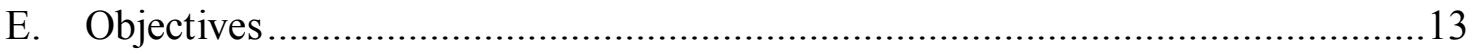

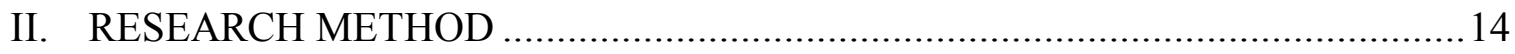

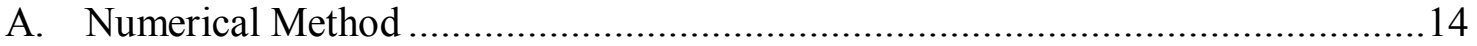

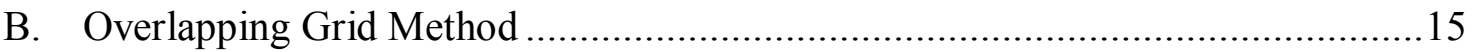

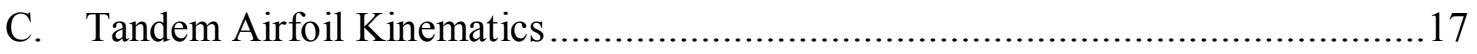

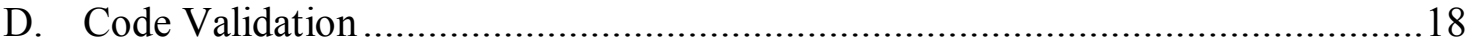

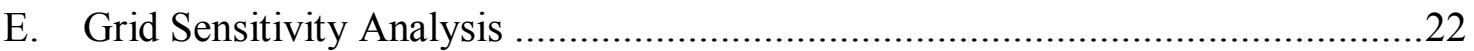

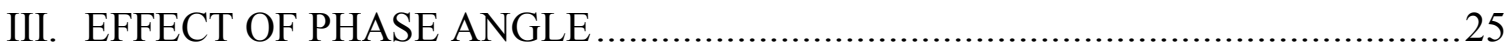

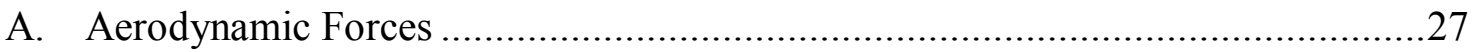

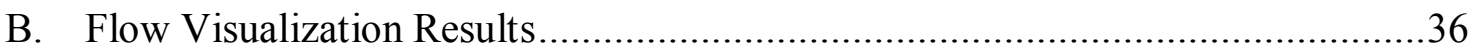

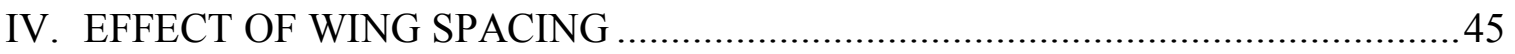

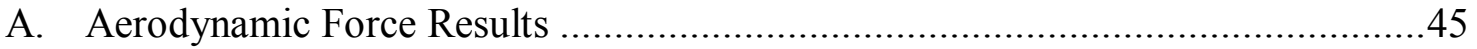

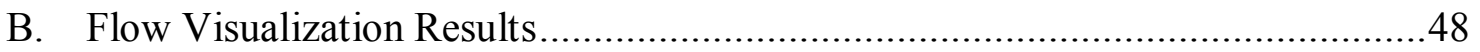

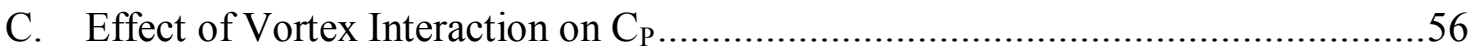

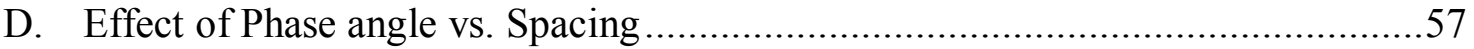

E. Cycle Averaged Force Results ....................................................................61 


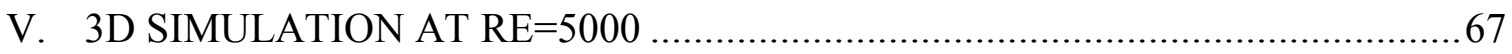

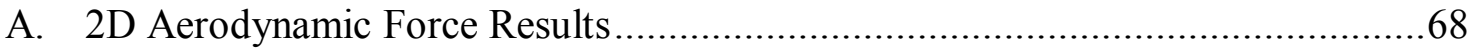

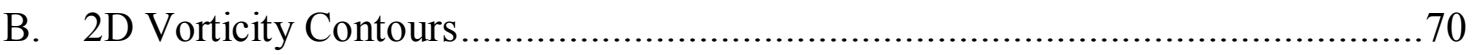

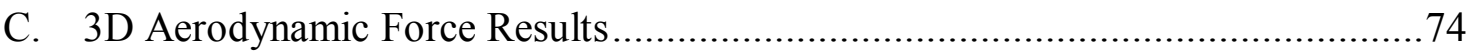

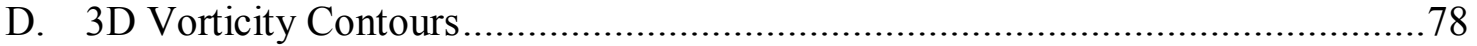

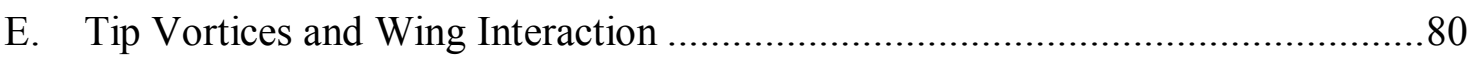

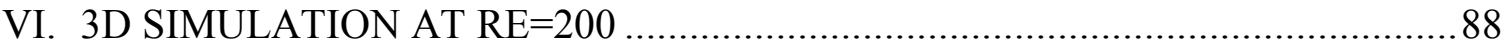

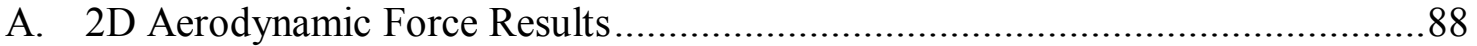

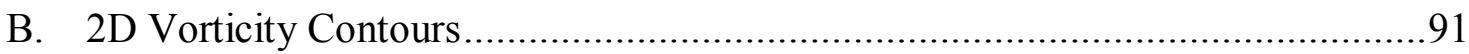

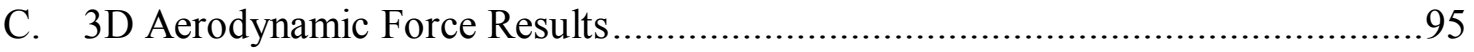

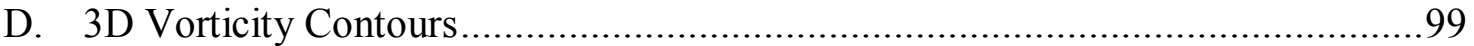

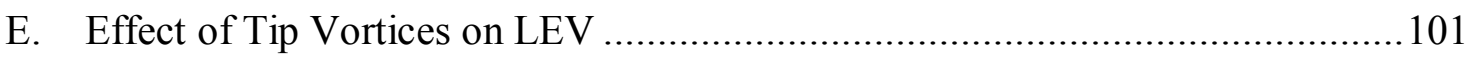

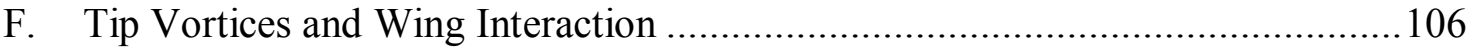

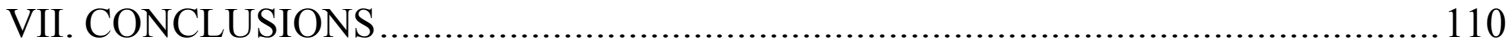

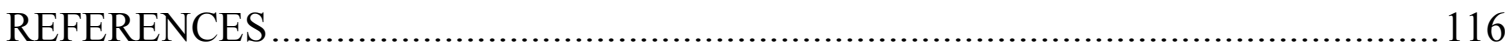

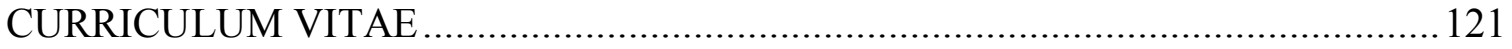




\section{LIST OF TABLES}

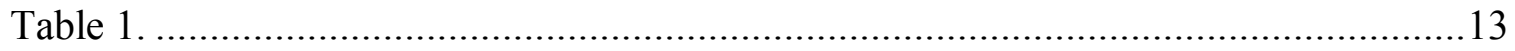

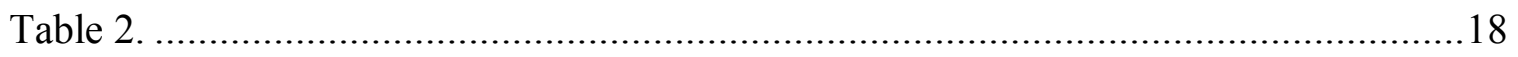

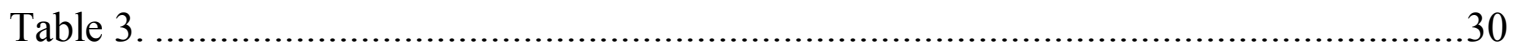

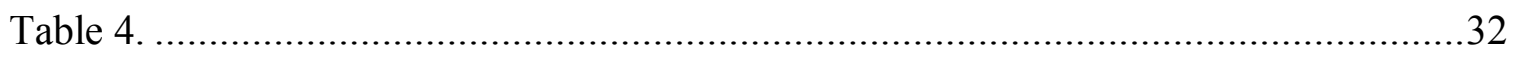

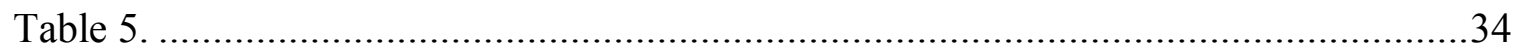

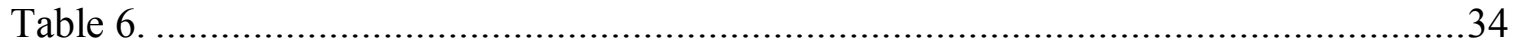

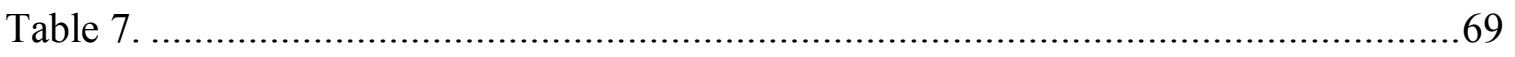

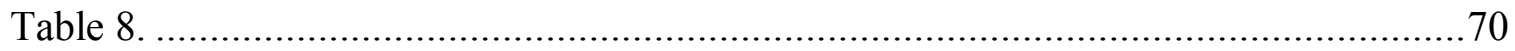

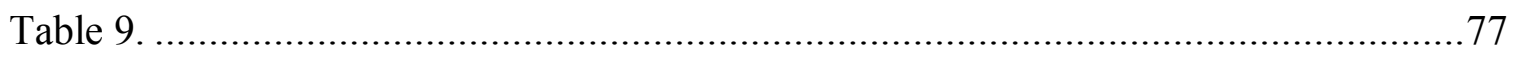

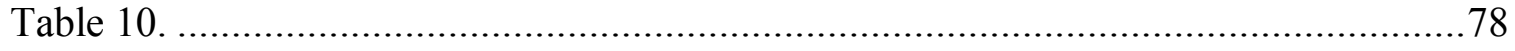

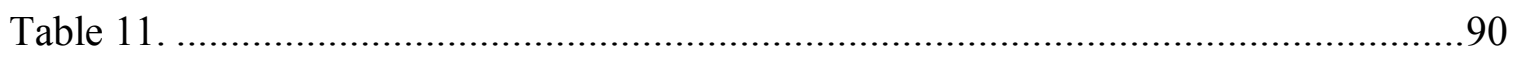

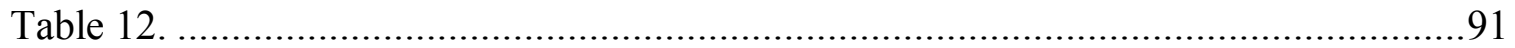

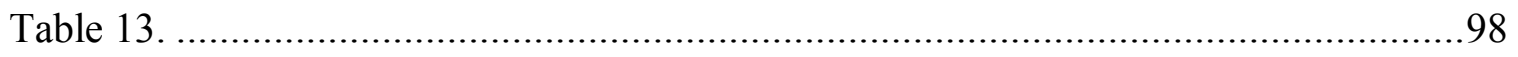

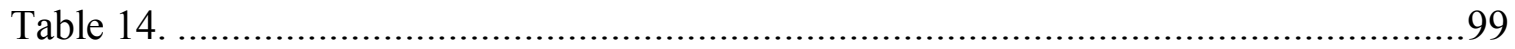




\section{LIST OF FIGURES}

Figure 1 .

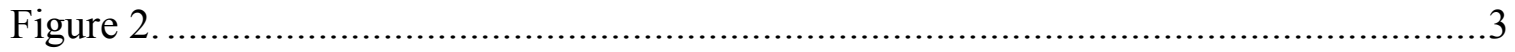

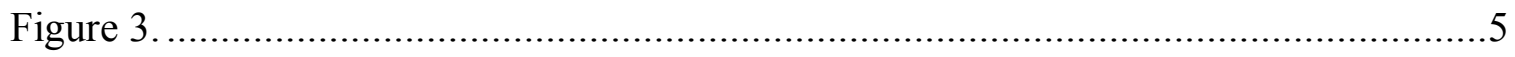

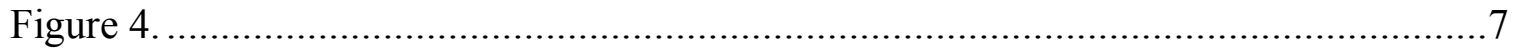

Figure 5 .

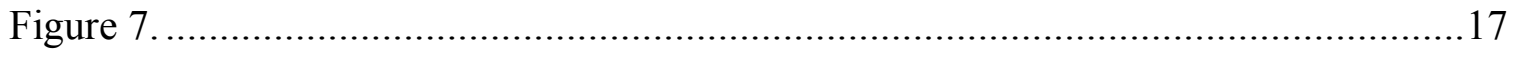

Figure 8

Figure 9

Figure 10,

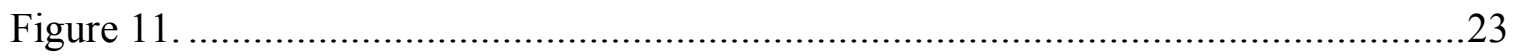

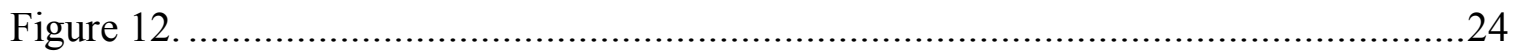

Figure 13.

Figure 14.

Figure 15 .

Figure 16 .

Figure 17.

Figure 18.

Figure 19.

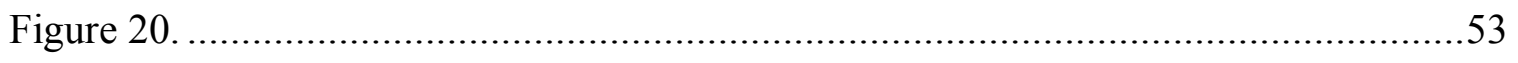

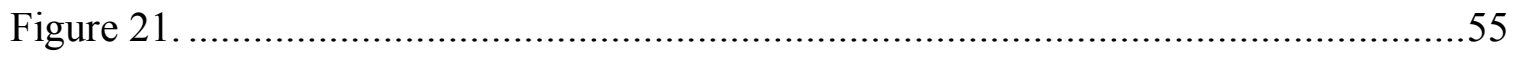

Figure 22.

Figure 23.

Figure 24.

Figure 25

Figure 26.

Figure 27.

Figure 28.

Figure 29.

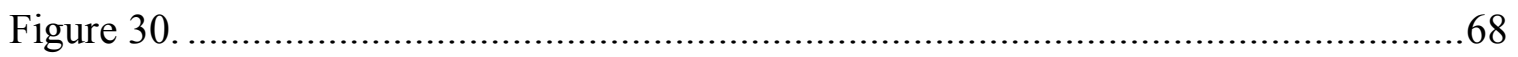

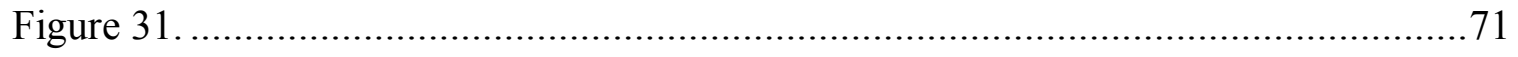

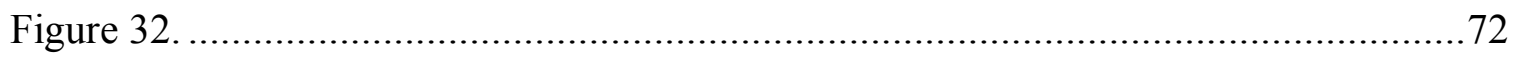

Figure 33.

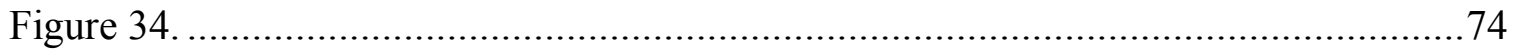


Figure $35 \ldots \ldots+1.0 .12$

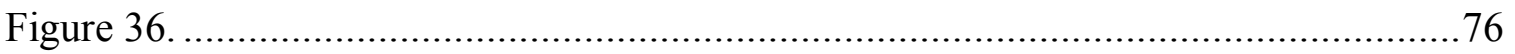

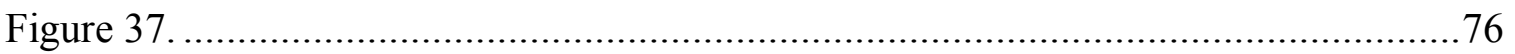

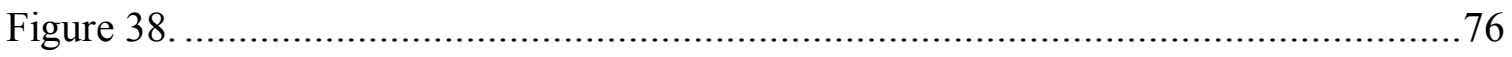

Figure 39.

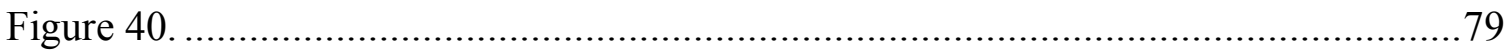

Figure 41 .

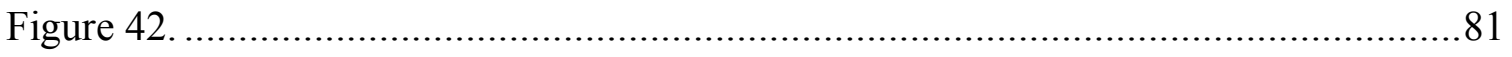

Figure 43

Figure 44 .

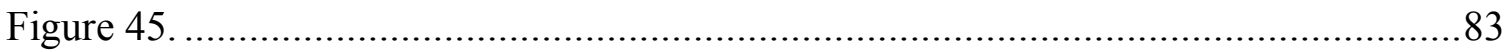

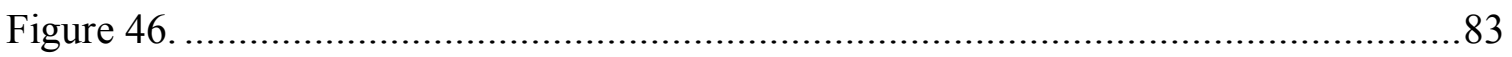

Figure 47.

Figure 48,

Figure 49.

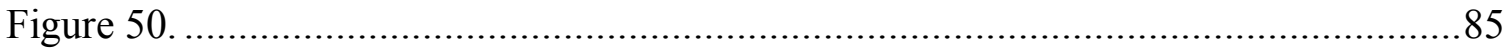

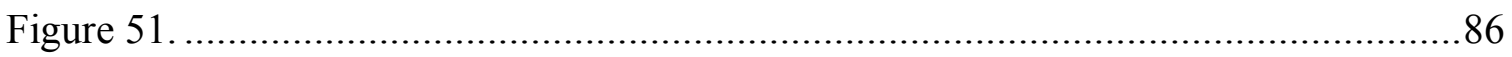

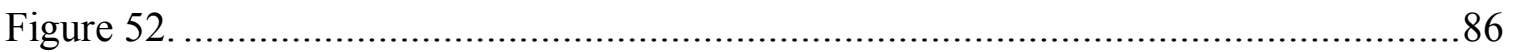

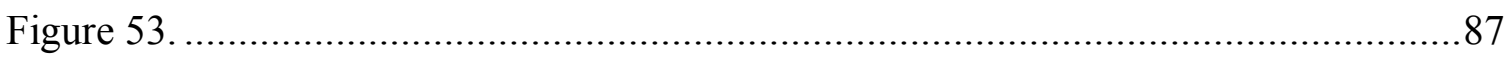

Figure 54

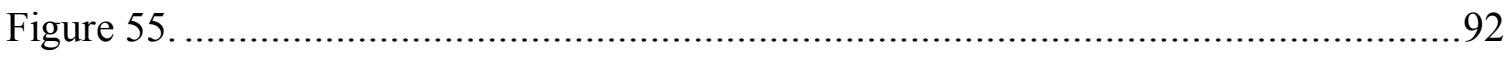

Figure 56

Figure 57.

Figure 58

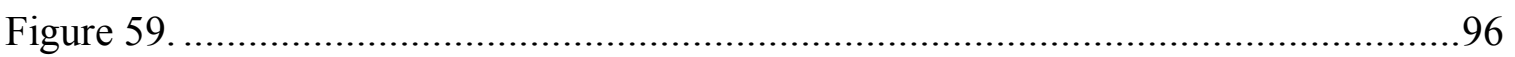

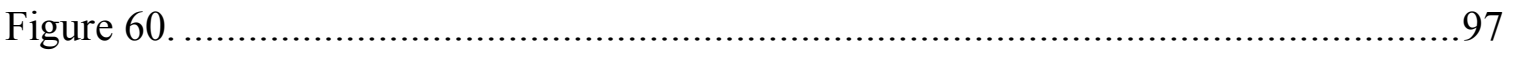

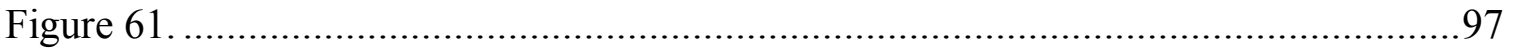

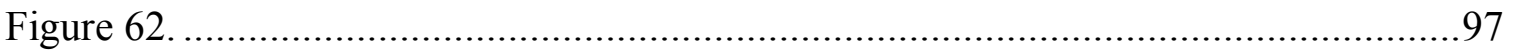

Figure 63

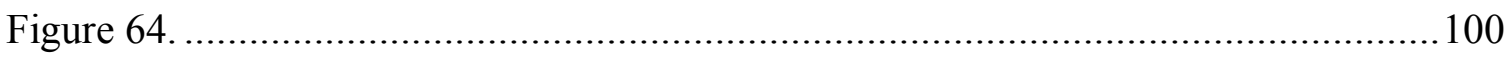

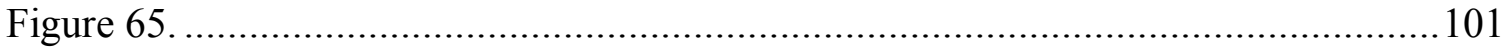

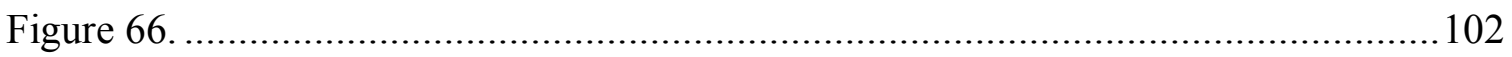

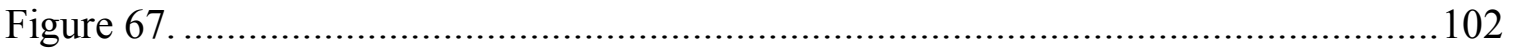

Figure 68 .

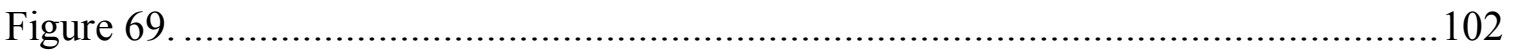

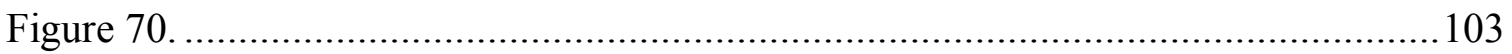

Figure $71 \ldots \ldots \ldots$

Figure 72,

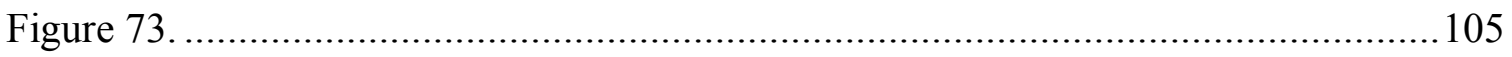

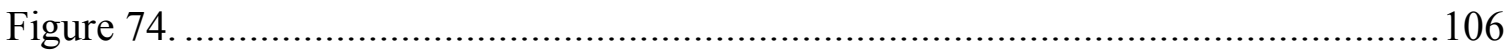




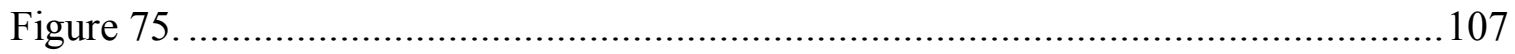

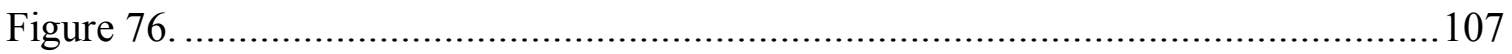

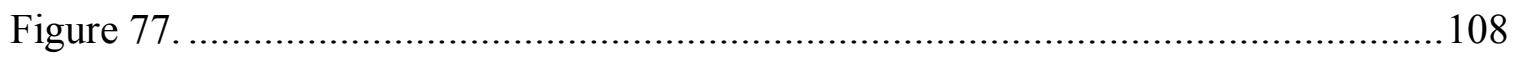


NOMENCLATURE

$\begin{array}{lll}A & = & \text { planform area with unit depth } \\ c & = & \text { wing chord length } \\ C_{L} & = & \text { lift coefficient } \\ C_{P} & = & \text { power coefficient } \\ C_{R} & = & \text { resultant coefficient } \\ C_{T} & = & \text { thrust coefficient } \\ f & = & \text { flapping frequency } \\ h_{0} & = & \text { plunging amplitude } \\ h(t) & = & \text { plunging displacement } \\ L & = & \text { instantaneous lift force } \\ M & = & \text { instantaneous pitching moment } \\ p & = & \text { pressure } \\ t & = & \text { time } \\ t_{0} & = & \text { time to reach } 99 \% \text { angular velocity } \\ T & = & \text { flapping period } \\ u & = & \text { flow velocity } \\ k & = & \text { reduced frequency } \\ S t & = & \text { Strouhal number } \\ V & = & \text { instantaneous plunge velocity } \\ \alpha_{a v e} & = & \text { average angle of attack } \\ \alpha_{0}= & \text { pitching amplitude } \\ \alpha(t) & = & \text { pitching angle } \\ \eta_{L} & = & \text { lift efficiency } \\ \eta_{P}= & \text { propulsive efficiency } \\ \eta_{R} & = & \text { resultant efficiency } \\ v & = & \text { kinematic viscosity } \\ \rho & = & \text { fluid density } \\ \varphi_{\alpha}= & \text { pitching phase lag } \\ \varphi_{h} & = & \text { plunging phase lag } \\ \psi & = & \text { phase angle } \\ \Omega_{0} & = & \text { maximum angular velocity } \\ & = & \end{array}$




\section{INTRODUCTION}

\section{A. Micro Air Vehicles}

The success in recent years of unmanned air vehicles, or UAV's has generated an interest in research efforts to create similar vehicles of reduced size. These micro air vehicles (MAVs) are small, autonomous air vehicles that are of interest for a variety of military and civilian applications. The term MAV is typically used to define an air vehicle that has no length dimension greater than 6 inches and a gross takeoff weight (GTOW) of less than $200 \mathrm{~g}$. Such tiny air vehicles would be capable of operating in confined spaces, making them ideal for missions in urban environments. Missions of primary interest include reconnaissance and surveillance, target detection, and search and rescue. Some examples of recent MAV designs include the Black Widow, designed by Aerovironment ${ }^{[1]}$, and the Microbat by Caltech ${ }^{[2]}$ both of which are shown in Figure 1. The Black Widow is a fixed wing MAV design that has a GTOW of $80 \mathrm{~g}$ and is capable of remaining airborne for almost 30 minutes, while the Microbat is a flapping wing MAV design that has a GTOW of $10 \mathrm{~g}$ and is capable of remaining airborne for less than 5 minutes. MAV's such as these have a number of potential uses, however, designing MAV's similar to these, with extended flight times and high maneuverability, presents a number of challenges. 

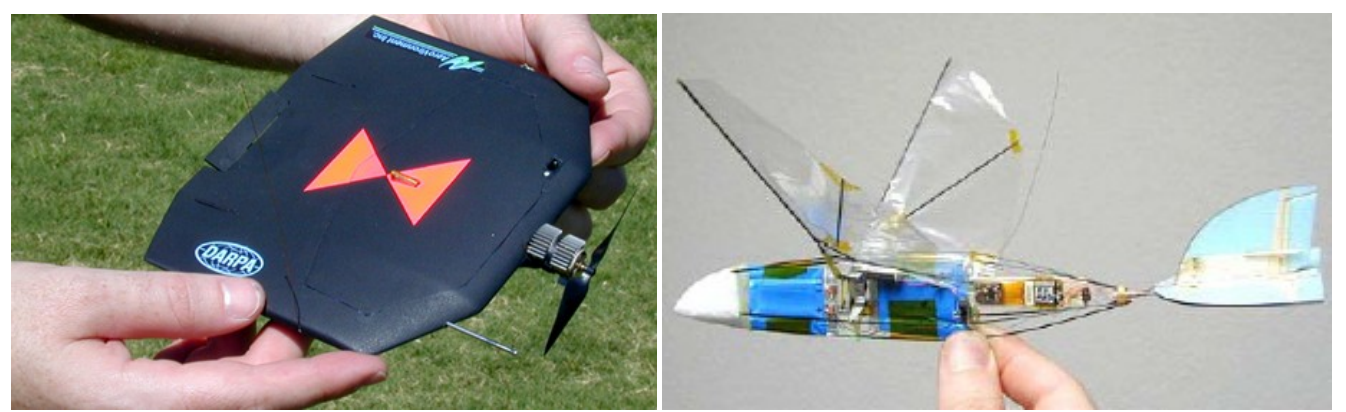

Figure 1. Two typical MAV designs. The Black Widow ${ }^{[1]}$ on the left and the Microbat ${ }^{[2]}$ on the right.

Due to their small size and slow flight velocity, MAV's operate at low Reynolds numbers, below 100,000, which presents a number of challenging aerodynamic problems such as massive laminar flow separation and laminar-to-turbulent transition. At low Reynolds numbers, flow across the wing is typically laminar and viscous effects become important. Conventional fixed wing aircraft are designed to operate at high Reynolds numbers, well above 100,000, where the flow is turbulent and remains attached. In contrast, laminar flow over the wing separates before the flow becomes turbulent, leading to early stalls. The decreased efficiency, in terms of the lift to drag ratio, of fixed wings at Reynolds numbers below 100,000, is shown in Figure $2^{[3]}$. All these challenges make designing fixed wing MAV's, aimed for operating over a wide range of angles of attack and extended flight time, challenging. Investigating nature, however, provides an alternative design method. Natural fliers, such as small birds and insects, operate in the same Reynolds number regime as MAV's, producing superior aerodynamic performance through flapping motions. 


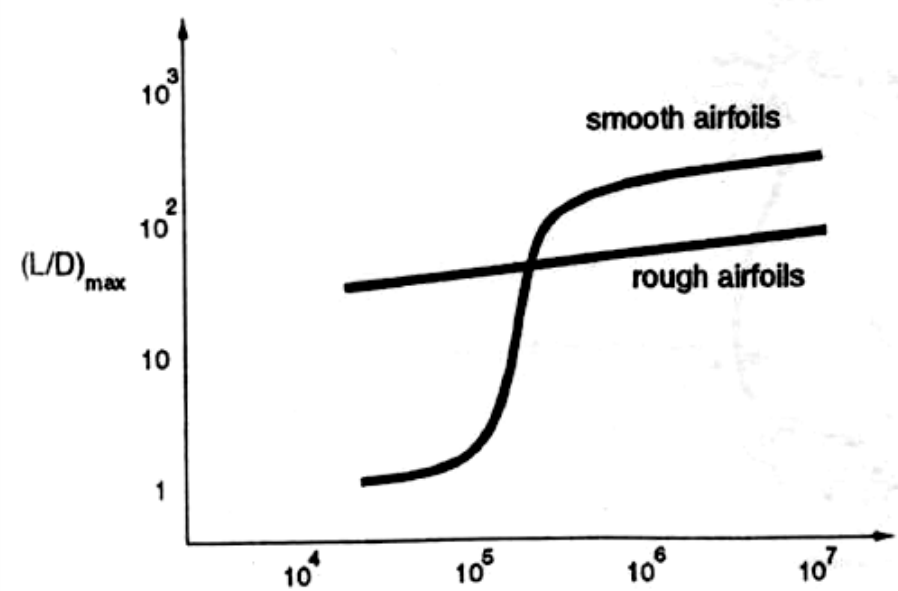

Figure 2. Lift coefficient vs Re for smooth and rough airfoils ${ }^{[3]}$.

Understanding the methods birds and insects use to attain high lift performance will provide useful insights in developing more efficient MAV designs. Flapping wings rely heavily on the generation and complex interactions of vortices to provide lift and thrust as opposed to the steady state flow dynamics utilized by fixed wing designs ${ }^{[4-6]}$. Of particular interest is the role leading edge vortices play in the enhancement of lift and thrust. Several unsteady mechanisms, which will be discussed later, have been proposed to explain the role of vortices in lift and thrust generation such as clap and fling, delayed stall, and wake capture.

\section{B. Flapping Wing Aerodynamics}

Flapping wing movement, similar to a bird or insect, is a complicated mix of periodic pitching (rotational motion), plunging (vertical motion) and surging (horizontal motion). The unsteadiness of flapping motions can be characterized by a dimensionless number called the reduced frequency

$$
k=\frac{\omega c}{2 u}
$$


where $\omega, c$, and $U$ are the flapping wing's angular velocity, the wing's reference chord, and the forward flight velocity respectively. The faster the wing flaps, or the slower the flight velocity, the higher value of the reduced frequency, and the greater the increase in unsteady effects. Smaller birds tend to have higher reduced frequencies than larger birds, indicating that they fly under more unsteady flow conditions ${ }^{[6]}$.

Another useful nondimensional parameter that can be used to characterize flight performance is the Strouhal number

$$
S t=\frac{2 f h_{a}}{u}
$$

where $f$ is the flapping frequency, $h_{a}$ is flapping amplitude, and $u$ is the flight speed. The Strouhal number is often used as a measure of flight efficiency. Studies by Taylor et al. ${ }^{[7]}$ show that the Strouhal number of 42 different species of bats, birds and insects in cruise flight fell within a narrow range of $0.2<\mathrm{St}<0.4$, with an average value of 0.29 . This indicates that the Strouhal number can be used as a guideline for optimizing flapping wing designs for efficiency.

Knoller and Betz ${ }^{[8,9]}$ are attributed as the first to observe that a plunging airfoil in a moving flow stream produces a net thrust. The relative vertical velocity imparted by the up and down stroke creates an effective angle of attack, which causes the resultant force to be slanted forward, indicating a thrust component. Figure 3 illustrates this for an airfoil at zero angle of attack. 


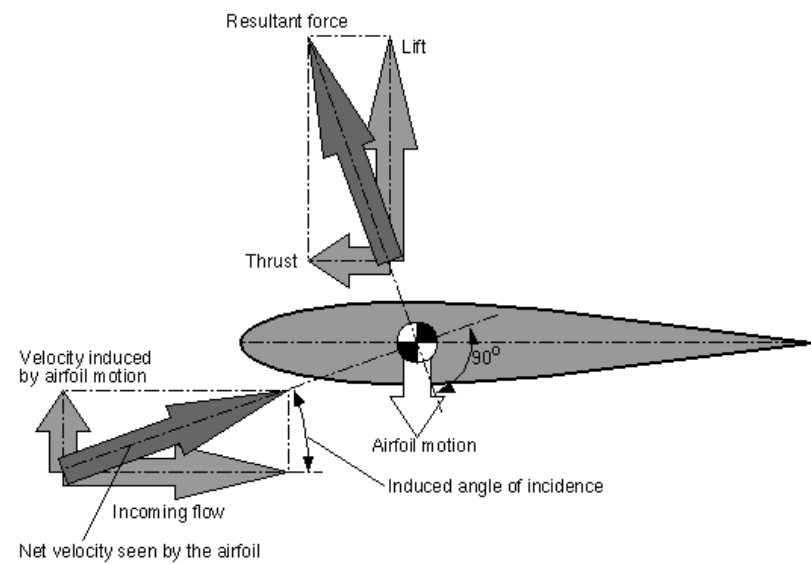

\section{Figure 3. Induced velocities and resultant forces on a plunging airfoil ${ }^{[10]}$.}

As described by Knoller and Betz, the plunging airfoil in this figure will generate a net thrust but no lift, since the negative lift component of the upstroke cancels the positive lift component of the downstroke. Lift generation requires that the plunging airfoil have a positive angle of attack. In this case, the effective angle of attack will be greater on the downstroke than the upstroke. Since lift increases with increasing angle of attack, up until a stall, the resultant force on the downstroke will be greater than the resultant force on the upstroke, therefore; the downstroke will produce more thrust than the upstroke and will generate a larger positive lift than the negative lift produced by the upstroke. In this case the plunging airfoil will generate net thrust and lift. The KnollerBetz model, however; fails to take into account the vortices that are generated and shed as the airfoil is flapped.

An airfoil undergoing pure pitching is also capable of producing thrust as first shown by Garrick ${ }^{[11]}$. The pitching frequency must be very high, however; for the thrust production to overcome the drag over the airfoil. This leads to the possibility, as observed in natural fliers, that the most efficient means of propulsion is a combination of pitching and plunging. Of particular interest in this case is what phase angle between 
pitching and plunging leads to the most efficient thrust generation. Investigations using panel code on a NACA 0012 airfoil pitching about its quarter chord point at an amplitude of $4^{\circ}$ have shown that a pitching-plunging mode is most efficient when pitching leads plunging by $90^{\circ}[12,13]$.

\section{Vortex Dynamics}

The vortex dynamics play a large part in the flight physics of flapping airfoils. Several mechanisms have been described to explain these vortices and the role they play in the generation of thrust and lift. Of special interest is the role of leading edge vortices in enhancing the thrust and lift. When leading edge vortices are formed they can increase the circulation over the top of the airfoil, causing a significant drop in pressure over the top of the airfoil and increasing lift ${ }^{[6,14]}$. The leading edge vortex travels along the top of the airfoil until it is shed into the wake where it can interact with the trailing edge vortex.

Dynamic stall is a mechanism proposed to explain the ability of both birds and insects are capable of attaining very high lift forces by delaying stall ${ }^{[6,14]}$. Leading edge vortices are utilized to sustain the lift of a rapidly pitching airfoil past the static stall limit. As the static stall limit is exceeded, the flapping airfoil gains a large increase in lift force. The stall is delayed thanks to the formation of a leading edge vortex, which sustains and enhances the lift even further. The vortex can persist for several chord lengths before convecting downstream, allowing the wing to maintain lift beyond the stall angle. When the wing pitches down, the lift does not immediately return to the pre-stall value until it reaches a much lower angle of attack resulting in hysteresis. This dynamic stall allows a flapping airfoil to sustain a higher angle of attack, for brief periods, than is possible for a fixed wing, leading to the generation of a greater lift force. 
The clap and fling method is observed in insects and was first proposed by WeisFogh while studying small wasps ${ }^{[15]}$. He observed that at the end of the upstroke and the beginning of the downstroke the two wings were brought together and then rapidly peeled apart. This maneuver causes large amounts of circulation, creating a very low pressure region over the wings and greatly enhances lift. Figure 4 is a schematic demonstrating this process.
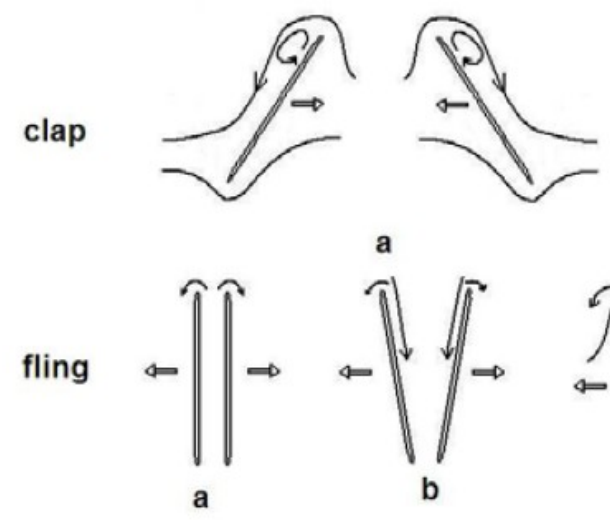

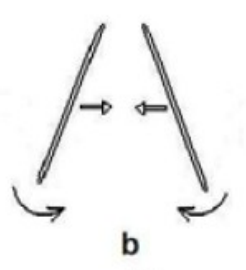

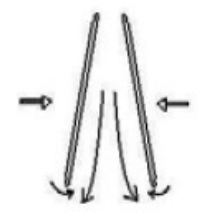

C

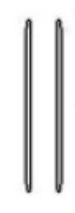

d

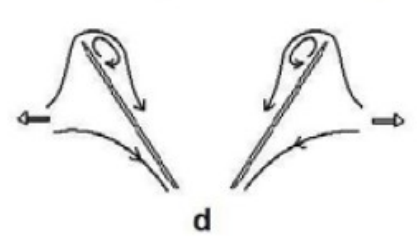

Figure 4. The clap and fling mechanism ${ }^{[14]}$.

While this process isn't performed continuously during flight, it is utilized for rapid flight maneuvers, and to attain high agility, such as a fly's ability to take off extremely quickly.

Wake capture is a mechanism that has been observed in natural fliers while hovering. This mechanism enhances lift through interactions between the wake and the airfoil $^{[6,14]}$. Figure 5 illustrates the process.
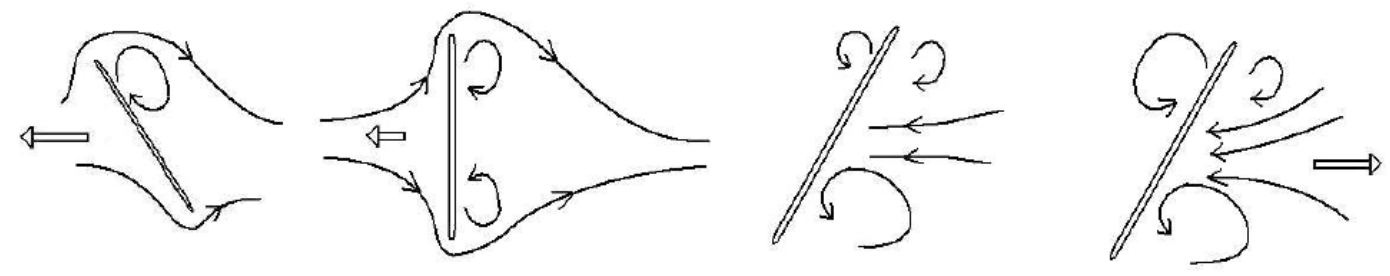

Figure 5. The wake capture mechanism ${ }^{[14]}$. 
During the upstroke, the wing sheds a vortex into the wake. The shed vortex meets the wing during the downstroke motion, causing a higher induced velocity. This causes a noticeable peak in the lift force and greatly aids the flier in sustained hovering.

\section{Tandem Wing Aerodynamics}

Studying the unsteady flow interactions between two closely situated airfoils is far more complex than the case of a single airfoil; however, two pairs of airfoils can provide a number of benefits such as increased lift and thrust, and improved flight stability ${ }^{[5]}$. Therefore, understanding these complex interactions can lead to improved MAV designs. Though there are several different ways of configuring two pairs of airfoils, of primary concern to this study are configurations similar to that of a dragonfly, where a pair of fore and hind wings are both flapping together.

Dragonflies are capable flying insects that utilize two pairs of independently actuated wings, with the hind set of wings operating in the wake of the fore set of wings. They are one of the fastest and most maneuverable flying insects, with measured flight speeds of up to $10 \mathrm{~m} / \mathrm{s}$ and instantaneous accelerations up to $4 \mathrm{~g}$ 's ${ }^{[16]}$. Tethered dragonflies have even been measured producing up to 20 times their body weight in lift

forces ${ }^{[17]}$. Their impressive flight capabilities have generated interest in the study of flapping tandem wing configurations as a basis for the design of micro air vehicles (MAVs) that operate at similar Reynolds numbers.

A significant trait of the tandem wing arrangement is that the hindwing interacts with the wake of the forewing. Experiments by Schmidt have shown that placing a nonflapping hindwing in the wake of a flapping forewing almost doubles the propulsive 
efficiency compared to the forewing flapping alone ${ }^{[18]}$. Similar results were obtained by Bosch through theoretical analysis and by Tuncer and Platzer using CFD analysis ${ }^{[19,20]}$.

Rather than employing a fixed set of hindwings, dragonflies flap both pairs of wings. Because both pairs of wings are independently actuated, the dragonfly can adjust the phase angle, $\psi$, between the flapping motion of the fore and hind wings. By observing dragonflies in flight, Alexander noted that they frequently make use of phase shifting; flapping inphase $\left(\psi=0^{\circ}\right)$ during takeoff or when undergoing maneuvers and flapping out of phase when in cruising flight ${ }^{[21]}$. Further observations by Ruppell ${ }^{[22]}$, Azuma and Watanabe ${ }^{[23]}$, and Thomas et al. ${ }^{[24]}$ have noticed similar behavior, and it has been postulated that flapping inphase allows for high force production while flapping out of phase allows for increased efficiency, with the hindwing extracting energy from the wake of the forewing ${ }^{[21,25]}$.

The correlation between the phase angle and flight mode in dragonflies has led to a number of studies concerning the relationship between the phase angle and the force production of tandem flapping wing configurations, both experimentally and computationally. Most studies have focused on tandem wings in hovering motion ${ }^{[26-32]}$. Results by Wang and Russell ${ }^{[29]}$ and Lan and Sun ${ }^{[30]}$ both show that the maximum resultant force is produced when the wings flap inphase, while Wang and Russell also show that the highest efficiency is achieved when the wings flap with a phase angle near $180^{\circ}$. Usherwood and Lehmann experimentally demonstrated that certain phase angles increase the efficiency of the tandem wing, but in their case, maximum efficiency was achieved when $\psi=90^{\circ}{ }^{[26]}$. They concluded that this increase in efficiency was due to the hindwing extracting energy from the wake of the forewing by removing swirl. 
Meanwhile, most of these studies show that the lift of both the fore and hind wings is noticeably reduced from that of a single wing at most phase angles ${ }^{[26,27,30,31]}$.

The relationship between force production and the phase angle has also been studied for tandem wings in forward flight experimentally ${ }^{[33-36]}$ and numerically ${ }^{[37-39]}$. Studies by both Akhtar, et al. ${ }^{[37]}$ and Warkentin and Delaurier ${ }^{[33]}$ showed that for certain phase angles, the propulsive efficiency of the tandem wing arrangement was almost double that of a single wing. This mirrors the results mentioned earlier by Schmidt ${ }^{[18]}$, Bosch ${ }^{[19]}$ and Tuncer and Platzer ${ }^{[20]}$. Both Huang and Sun ${ }^{[38]}$ and Wang and Sun ${ }^{[39]}$ simulated 3D tandem wings at different phase angles and advance ratios, using a Navier Stokes solver. Huang and Sun found that at all advance ratios, the lift and thrust coefficients of the tandem wing case were nearly constant and equal to a single wing when the hindwing led the forewing, but when the forewing led the hindwing, they found that the lift and thrust coefficients were noticeably reduced ${ }^{[38]}$. Wang and Sun, however, demonstrated that the resultant force coefficient of the tandem configuration was noticeably lower than a single wing at most of the tested phase angles at all advance ratios ${ }^{[39]}$. At each advance ratio, however; the resultant force coefficient nearly equaled that of a single wing at one of the tested phase angles, which increased from $0-90^{\circ}$ as the advance ratio increased.

The dynamic by which the phase angle affects force production is often associated with variations in the wing vortex interactions between the fore and hind wings. Work by Saharon and Luttgess with a robotic tandem wing configuration showed that adjustments in the phase angle caused variations in the vortex interactions between the fore and hind wings ${ }^{[34-36]}$. While they did not measure force data, they hypothesized that the variations 
in the vortex interactions could influence force generation. Such variation in the vortex interactions has been linked to changes in the force production by other sources ${ }^{[27,37,40]}$. Variation in force generation due to vortex interactions with the hindwing would suggest that other parameters could affect force generation similarly to changes in the phase angle. Changes in the wing spacing and advance ratio could both potentially alter the point in the flapping cycle that the hindwing interacts with vortices shed from the forewing and affect the force generation. Wang and Sun ${ }^{[39]}$ showed that the phase angle at which the resultant force peaked changed as the advance ratio was increased while Maybury and Lehmann ${ }^{[27]}$ saw that the phase angle at which peak lift production occurred changed as the fore and hind wings were moved closer together; though neither of the two studies attempted to link these changes in the force production specifically to altered vortex interactions. Broering et al. ${ }^{[40]}$ linked the variation in force production of the hindwing to different vortex interactions between the fore and hind wings that altered the LEV generated by the hindwing at different phase angles. It was also observed by Rival et al. ${ }^{[41]}$ that certain vortex interaction not only increased thrust but also allowed the hindwing to extract energy from the forewing. Finally, Lim and Tay simulated a tandem configuration in forward flight and different phase angles as well as different spacings between the fore and hind wings. They demonstrated that at an optimum spacing and phase angle, the tandem wing has better performance than the single wing. They also described how variations in vortex interactions between the two wings affected the force coefficients and efficiencies ${ }^{[42]}$.

Table 1 shows a summary of some of the different studies that have analyzed the phase relationship between tandem flapping wings. 


\begin{tabular}{|c|c|c|}
\hline Group & Method & Results \\
\hline $\begin{array}{l}\text { Thomas, et } \\
\text { al.[24] }\end{array}$ & $\begin{array}{l}\text { Smoke } \\
\text { visualization/field } \\
\text { observation }\end{array}$ & $\begin{array}{l}\text { Counterphase stroke for cruise flight; inphase for } \\
\text { high maneuver and escape }\end{array}$ \\
\hline Lan [25] & Double Lattice & $\begin{array}{l}\text { Increase in thrust and efficiency at some optimum } \\
\text { phase angle when hindwing leads forewing }\end{array}$ \\
\hline $\begin{array}{l}\text { Lan and Sun } \\
{[30]}\end{array}$ & $\begin{array}{l}\text { 2D computational } \\
\text { (Forward Flight) }\end{array}$ & $\begin{array}{l}\text { Increase in thrust at } 90^{\circ} \text {, decrease in resultant at } 90^{\circ} \\
\text { and } 180^{\circ} \text {, resultant unchanged at } 0^{\circ}\end{array}$ \\
\hline $\begin{array}{l}\text { Sun and Lan } \\
{[31]}\end{array}$ & $\begin{array}{l}\text { 3D computational } \\
\text { (Hovering) }\end{array}$ & $\begin{array}{l}\text { Interaction between fore and hind wings detrimental, } \\
\text { reduced lift around both fore and hind wings. }\end{array}$ \\
\hline $\begin{array}{l}\text { Huang and } \\
\text { Sun [38] }\end{array}$ & $\begin{array}{l}\text { 3D computational } \\
\text { (Forward Flight) }\end{array}$ & $\begin{array}{l}\text { Lift and thrust forces decreased when forewing leads } \\
\text { hindwing, lift and thrust forces mostly unchanged } \\
\text { when hindwing leads forewing }\end{array}$ \\
\hline $\begin{array}{l}\text { Usherwood } \\
\text { and Lehmann } \\
{[26]}\end{array}$ & $\begin{array}{l}\text { Experimental } \\
\text { (Hovering) }\end{array}$ & $\begin{array}{l}\text { Interaction between fore and hind wings decreases } \\
\text { lift, } 22 \% \text { greater efficiency than isolated wings when } \\
\text { hindwing leads forewing by } 90^{\circ}\end{array}$ \\
\hline Schmidt [18] & Experimental & $\begin{array}{l}\text { Placing a fixed hind-wing in the wake of a flapping } \\
\text { fore-wing doubles the propulsive efficiency. }\end{array}$ \\
\hline Bosch [19] & Theoretical & $\begin{array}{l}\text { The propulsive efficiency is increased when a fixed } \\
\text { hind-wing is placed in the wake of a flapping fore } \\
\text { wing. }\end{array}$ \\
\hline $\begin{array}{l}\text { Tuncer and } \\
\text { Platzer [20] }\end{array}$ & $\begin{array}{l}\text { 2D computational } \\
\text { (forward flight) }\end{array}$ & $\begin{array}{l}\text { A fixed hind-wing, placed in the wake of a flapping } \\
\text { fore-wing, can increase propulsive efficiency. }\end{array}$ \\
\hline $\begin{array}{l}\text { Alexander } \\
{[21]}\end{array}$ & $\begin{array}{l}\text { Biological } \\
\text { observation }\end{array}$ & $\begin{array}{l}\text { Dragonflies flap inphase during high force } \\
\text { maneuvers and flap out of phase during cruising } \\
\text { flight. }\end{array}$ \\
\hline Ruppell [22] & $\begin{array}{l}\text { Biological } \\
\text { observation }\end{array}$ & $\begin{array}{l}\text { Dragonflies make use of phase shifting during } \\
\text { winged flight. }\end{array}$ \\
\hline $\begin{array}{l}\text { Azuma and } \\
\text { Watanabe } \\
\text { [23] }\end{array}$ & $\begin{array}{l}\text { Biological } \\
\text { observation }\end{array}$ & $\begin{array}{l}\text { Phase shifting between the fore and hind wings is } \\
\text { utilized by dragonflies during flight. }\end{array}$ \\
\hline $\begin{array}{l}\text { Wang and } \\
\text { Russell [29] }\end{array}$ & $\begin{array}{l}\text { 2D computational } \\
\text { (hovering) }\end{array}$ & $\begin{array}{l}\text { Maximum resultant force achieved when flapping } \\
\text { inphase. Maximum efficiency achieved when } \\
\text { flapping with a phase angle near } 180^{\circ} \text {. }\end{array}$ \\
\hline Akhtar [37] & $\begin{array}{l}\text { 2D computational } \\
\text { (forward flight) }\end{array}$ & $\begin{array}{l}\text { At certain phase angles the propulsive efficiency of a } \\
\text { tandem configuration is nearly double that of a } \\
\text { single wing. }\end{array}$ \\
\hline $\begin{array}{l}\text { Warkentin } \\
\text { and Delaurier } \\
\text { [33] }\end{array}$ & $\begin{array}{l}\text { Experimental } \\
\text { (forward flight) }\end{array}$ & $\begin{array}{l}\text { Tandem configuration has higher propulsive } \\
\text { efficiency than single wing at certain phase angles. }\end{array}$ \\
\hline $\begin{array}{l}\text { Wang and } \\
\text { Sun [39] }\end{array}$ & $\begin{array}{l}\text { 3D computational } \\
\text { (forward flight) }\end{array}$ & $\begin{array}{l}\text { The resultant force coefficient of the tandem } \\
\text { configuration at different phase angles is noticeably } \\
\text { lower than that of a single wing. }\end{array}$ \\
\hline
\end{tabular}




\begin{tabular}{|l|l|l|}
\hline $\begin{array}{l}\text { Saharon and } \\
\text { Luttgess [34- }\end{array}$ & Experimental & $\begin{array}{l}\text { Adjusting the phase angle affects the vortex } \\
\text { interactions between the fore and hind wings. }\end{array}$ \\
\hline $\begin{array}{l}\text { Maybury and } \\
\text { Lehmann } \\
{[27]}\end{array}$ & $\begin{array}{l}\text { Experimental } \\
\text { (hovering) }\end{array}$ & $\begin{array}{l}\text { The phase angle at which peak lift production occurs } \\
\text { changes as the hind-wing is moved closer to the fore- } \\
\text { wing }\end{array}$ \\
\hline $\begin{array}{l}\text { Lim and Tay } \\
\text { [42] }\end{array}$ & $\begin{array}{l}\text { 2D computational } \\
\text { (forward flight) }\end{array}$ & $\begin{array}{l}\text { At certain spacing and phase angle the tandem wing } \\
\text { performs better than a single wing. }\end{array}$ \\
\hline $\begin{array}{l}\text { Rival et al. } \\
{[41]}\end{array}$ & Experimental & Fore-wing is not affected by the hind-wing \\
\hline
\end{tabular}

Table 1. Summary of studies investigating the phase relationship between tandem wings.

\section{E. Objectives}

The objectives for the current study are as follows.

- Develop a computational model to study tandem flapping wing aerodynamics using an incompressible Navier-Stokes solver on composite overlapping grids.

- Characterize the vortex interactions between the fore and hind wing and determine its effect on lift, thrust and efficiency.

- Determine the effect of phase angle and wing spacing between the fore and hind wings on the vortex interactions and the resulting lift, thrust and efficiency.

- Evaluate the effect of vortex shedding on the performance of the hindwing by comparing to the performance of a single wing.

- Compare the performance of the tandem configuration on a systems level (combined fore and hind wings) to the performance of a single wing. 


\section{RESEARCH METHOD}

\section{A. Numerical Method}

The flow field is described by the unsteady incompressible form of the NavierStokes equations written in primitive-variables

$$
\begin{gathered}
u_{t}+(u \cdot \nabla) u+\nabla p=\nu \triangle u \\
\nabla \cdot u=0
\end{gathered}
$$

where $\mathrm{u}$ is the flow velocity, $\mathrm{p}$ is the kinematic pressure, $v$ is the kinematic viscosity and $\Delta$ represents the Poisson operator. To avoid the checker-board instability problem associated with the direct discretization of the pressure term, the above equations are rewritten in the following so called "velocity-pressure" formulation ${ }^{[43]}$,

$$
\begin{gathered}
u_{t}+(u \cdot \nabla) u+\nabla p-\nu \triangle u=0 \\
\triangle p-\left(\nabla u \cdot u_{x}+\nabla v \cdot u_{y}+\nabla w \cdot u_{z}\right)-C_{d}(\nu) \nabla \cdot u=0
\end{gathered}
$$

The new formulation is solved using the split-step approach which decouples the solution of the velocity variables from the solution of the pressure. In the velocitypressure formulation the term $C_{d}(\nu) \nabla \cdot u$ is added to damp the divergence. Spatial discretization was carried out over composite overset computational grids using a second order accurate central difference. Time integration was through an Adams-BashforthMoulton predictor-corrector method. For the predictor step we use a semi-implicit scheme which discretizes the viscous terms using a Crank-Nicholson treatment and the convection terms using an Adam-Bashforth predictor-corrector. The predictor step is 


$$
\frac{u^{p}-u^{n}}{\triangle t}=\frac{3}{2} f_{E}^{n}-\frac{1}{2} f_{E}^{n-1}+\alpha A u^{p}+(1-\alpha) A u^{n}
$$

and the Adams-Moulton corrector step is

$$
\frac{u^{c}-u^{n}}{\triangle t}=\frac{1}{2} f_{E}^{p}-\frac{1}{2} f_{E}^{n}+\alpha A u^{c}+(1-\alpha) A u^{n}
$$

where superscripts $p$ and $c$ represent the predicted and corrected values, respectively, and $f_{E}=-(u \cdot \nabla) u-\nabla p$ and $A u=\nu \triangle u . \quad \alpha$ was set to 0.5 , which gives a second order Crank-Nicolson method. An iterative solver, PETSc, is used to solve the discretized system of equations ${ }^{[44]}$.

For the Reynolds number studied, 5000, the flow was assumed to be laminar and no turbulence model was employed. While a Reynolds number of 5000 is too high to be considered purely laminar, several studies show only small differences in the force histories when using a laminar model compared to a turbulent model at Reynolds numbers below $60,000^{[45-48]}$.

The code was simulated using a microway server with 32 AMD Opteron processors at $2.0 \mathrm{GHz}$ and $32 \mathrm{~GB}$ of memory. Due to code limitations each case was limited to running on a single processor. The $2 \mathrm{D}$ cases used approximately 30,000 grid points and required a runtime of three hours in order to simulate seven flapping cycles. The 3D cases used approximately 2,000,000 grid points and required a runtime of 900 hours to simulate four flapping cycles.

\section{B. Overlapping Grid Method}

The wing flapping motion leads to a moving boundary problem. A moving grid approach is needed to dynamically update the computational grid to accommodate the

wing motion and so an overlapping moving grid method is adopted ${ }^{[49]}$. This method enables the use of boundary-conforming structured grids in order to achieve high quality 
representation of the boundaries associated with the airfoil surface while still allowing the use of Cartesian grids to represent the flow field so that the efficiencies inherent to such grids can be exploited.

In the overlapping grid method, interpolation points are located in the overlapping region between different grids and are used to couple the solutions. As the body moves, the grid associated with the body moves with it, meaning that only the interpolation points between overlapping grids must be recalculated as opposed to the need to regenerate the whole mesh.

Figure 6 shows a schematic of the overlapping grid used for the tandem wing analysis. An O-type grid was generated around the airfoil using a hyperbolic grid generation technique. A high resolution wake grid was used to capture the wake structures between the airfoils. The fine wake grid and background grids are all of uniform density. The airfoils have the smallest grid spacing, with each subsequent grid having a larger grid spacing up to the coarse background grid which has the largest grid spacing. The entire domain is 20 chord lengths in the $\mathrm{x}$ and $\mathrm{y}$ directions with the tandem configuration centered in the domain. For the inlet boundary on the left a Dirichlet boundary condition $\left(u=U_{0}\right)$ was assigned while on the right side, as well as the top and bottom, a zero gauge pressure outlet condition was used. 


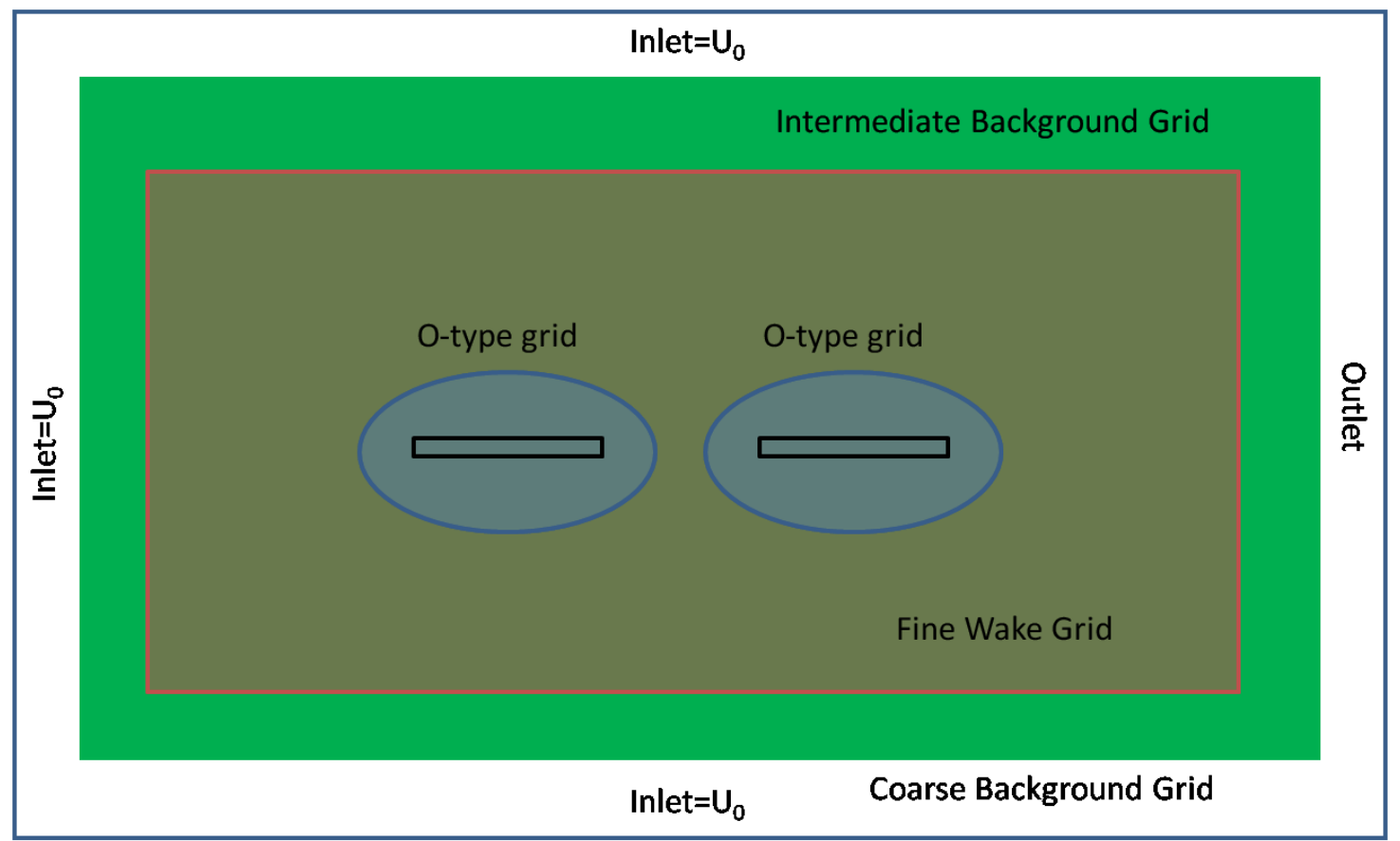

Figure 6. Schematic of the computational grid and boundary conditions used in the study. (Not shown to scale)

\section{Tandem Airfoil Kinematics}

The flapping kinematics used in the study was a combination of sinusoidal pitching and plunging, with the pitch axis at $0.25 \mathrm{c}$ from the leading edge. It was not the intent of the study to exactly replicate dragonfly kinematics, which can vary widely depending on the flight mode and trajectory ${ }^{[50]}$, but rather to study a tandem configuration undergoing simple periodic motion to reveal the pertinent wing/vortex and vortex/vortex interaction features. The flapping kinematics used were

$$
\begin{gathered}
\alpha(t)=\alpha_{0} \cos \left(2 \pi f t+\phi_{\alpha}+\phi_{h}\right)+\alpha_{a v e} \\
h(t)=h_{0} \cos \left(2 \pi f t+\phi_{h}\right)
\end{gathered}
$$

where $\alpha(t)$ is the pitching angle, $h(\mathrm{t})$ is the plunging displacement, $t$ is time, $f$ is the flapping frequency, $\alpha_{0}$ is the pitching amplitude, $h_{0}$ is the plunging amplitude, $\varphi_{\alpha}$ is the phase for pitch, $\alpha_{\text {ave }}$ is the average angle of attack, and $\varphi_{h}$ is the phase for plunge. The 
specific value of each parameter used for the single, fore and hind wings are shown in Table 2. Note that three possible values are used for $\varphi_{h}$ for the hind wing, representing the three different phase angles tested.

\begin{tabular}{|l|l|l|}
\hline Parameter & Single/Fore & Hind \\
\hline$\alpha_{0}$ & $20^{\circ}$ & $20^{\circ}$ \\
\hline$f$ & $0.3 \mathrm{~Hz}$ & $0.3 \mathrm{~Hz}$ \\
\hline$\varphi_{\alpha}$ & $90^{\circ}$ & $90^{\circ}$ \\
\hline$\alpha_{\text {ave }}$ & $5^{\circ}$ & $5^{\circ}$ \\
\hline$h_{0}$ & $0.5 \mathrm{c}$ & $0.5 \mathrm{c}$ \\
\hline$\varphi_{h}$ & $0^{\circ}$ & $0^{\circ}, 90^{\circ}, 180^{\circ}$ \\
\hline$S t$ & 0.3 & 0.3 \\
\hline$k$ & 0.942 & 0.942 \\
\hline
\end{tabular}

Table 2. Values used for the different parameters in the kinematic equations.

The chosen frequency and plunge amplitude result in a Strouhal number of 0.3 . The phase angle between pitch and plunge, $\varphi_{\alpha}$, was chosen to be $90^{\circ}$ (pitch leading plunge) which has been shown to be most efficient for flapping wings from a number of sources ${ }^{[12,13]}$. The average angle of attack was set such that a moderate amount of cycle averaged lift was produced. The inlet velocity, $u$, was set equal to one chord length per second, resulting in a chord-based Reynolds number of 5000, which is in the middle range of Reynolds number for dragonflies. The flapping Reynolds number, defined as $2 \pi f h_{0} c / v$, was equal to 4712 . The kinematic parameters used for the single wing are the same as those used for the fore and hind wings.

\section{Code Validation}

The case of a dynamic stall was studied in order to establish the validity of the code for the numerical simulation of moving boundary problems. The dynamic stall case has been extensively studied, both numerically and experimentally, and has a comprehensive database with which to compare ${ }^{[51-53]}$. In the dynamic stall case, a 
NACA0012 airfoil rapidly pitches up to generate a leading edge vortex, which convects over the top of the airfoil, creating a low pressure region and delaying the stall.

The dynamic stall was simulated at a chord-based Reynolds number of 10,000, with the airfoil rotating about the quarter chord position. The angular velocity of the airfoil was modeled with the following ramp function ${ }^{[52]}$

$$
\Omega(t)=\Omega_{0}\left(1-e^{-4.6 t / t_{0}}\right)
$$

where $\Omega_{0}$ is the maximum angular velocity, which was $0.2 \mathrm{rad} / \mathrm{s}$ and $t_{0}$ is the time taken, after the start of motion, to reach $99 \%$ of the maximum angular velocity, which was set to 0.5 seconds. In the simulation the airfoil was held in place to allow flow to become established before the airfoil was set in motion.

In the simulation, the Dirichlet boundary condition was set on the left side of the domain (inlet) on which the velocity was set as the freestream velocity; the pressure was prescribed on the right side of the domain (the outlet); the top and bottom boundaries were set as slip walls to simulate an infinite domain. The dynamic stall was modeled with different background domain sizes in order to determine the size needed to eliminate the wall effects. Three different domain sizes were tested, 10-chord by 10-chord, $20 \times 20$ and 40x40. The result shows that the differences among the three domain sizes are not significant, and there is only a minor difference in the maximum lift coefficient achieved between the three grids. The 20x20 and 10x10 grids could be used without altering the results significantly from the $40 \times 40$ grid. In our study, the 20x20 domain size is used.

The effect of different airfoil grid resolutions was also tested. Three different grid resolutions were tested, 192x96, 256x128, and 320x160. These airfoil grid resolutions were tested over two different background grid resolutions, 200x200 and 300x300. The 
results from the 200x200 grid are shown in Figure 7 and the results from the 300x300 grid, along with the computational results of a similar case from Liu and Kawachi ${ }^{[53]}$ and experimental results from McCroskey et al. ${ }^{[51]}$, are shown in Figure 8. Figure 7 shows that refining the airfoil grid has little effect on the results at the early stages of dynamic stall, up until $25^{\circ}$ angle of attack, but approaching the stall angle, grid refinement causes a noticeable difference in dynamic stall behavior. For the coarse grid, stall occurs at about 37 degrees and lift shows a sudden drop beyond the point. However, for the finer grids, the first stall occurs at a lower angle of attack. Instead of showing a sudden drop, the lift varies slowly with the angle of attack until the angle of attack reaches the second stall angle at a much higher angle of attack. After the second stall, the lift shows a sudden drop. Overall, the results show good convergence with airfoil grid refinement in regards to the upward slope, the first stall angle and the downward slope, however; there is no clear convergence of the second stall angle. It should also be pointed out that the lift coefficient spikes from 0 to about 1 at $\mathrm{t}=0$. This discontinuity is caused by the initial startup motion of the grid.

Figure 8 shows the results for the $300 \times 300$ background grid. Compared to the 200x200 background grid, the results show no change for the 192x96 and 256x128 grids, but show a noticeable difference for the 320x160 grid. The results for this background grid show good convergence as the airfoil grid is refined from $256 \times 128$ to $320 \times 160$. Overall the computational results in Figure 8 show similar behavior compared to the results from Liu and Kawachi ${ }^{[53]}$ and McCroskey et al. ${ }^{[51]}$ in terms of the upward and downward slopes. However, the stall angles differ from each other. It should be noted here that the experiment was performed at a much higher Reynolds number. Liu and 
Kawachi's simulation was conducted at the Reynolds number of 10,000 but his solution only shows one stall angle followed by a slow drop in the lift.

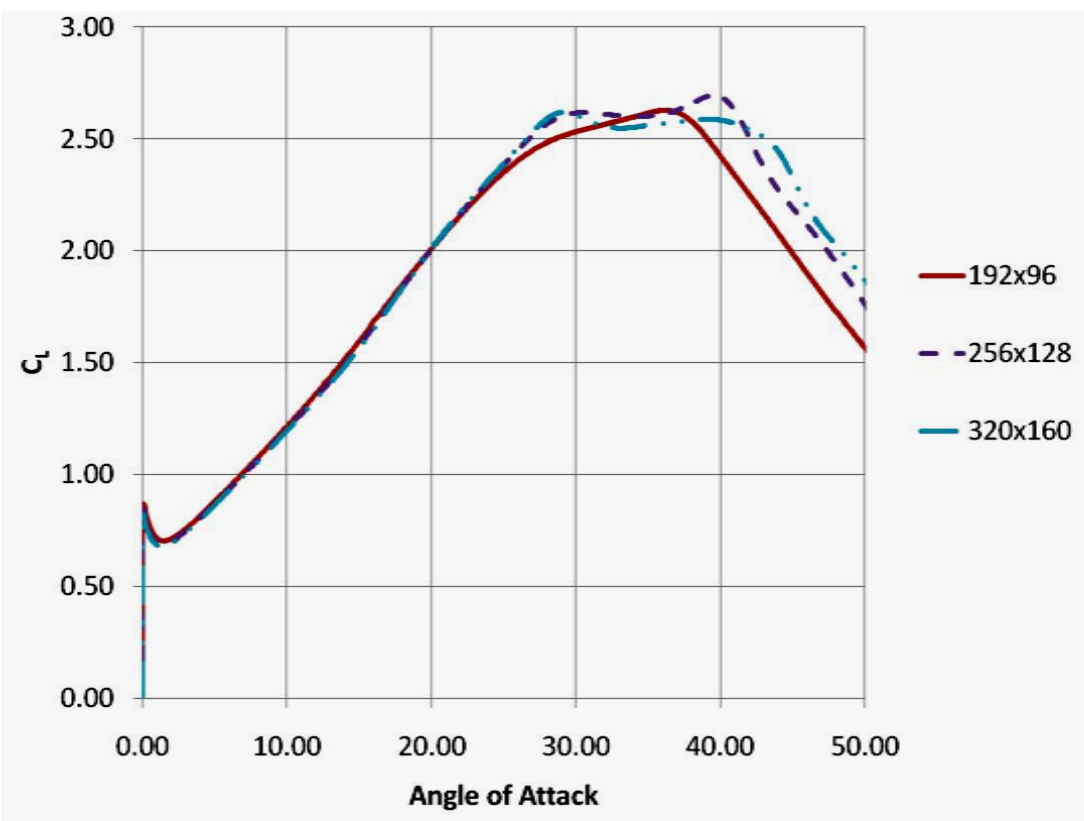

Figure 7. The lift coefficient versus angle of attack for three different airfoil grid resolutions on a $200 \times 200$ background grid.

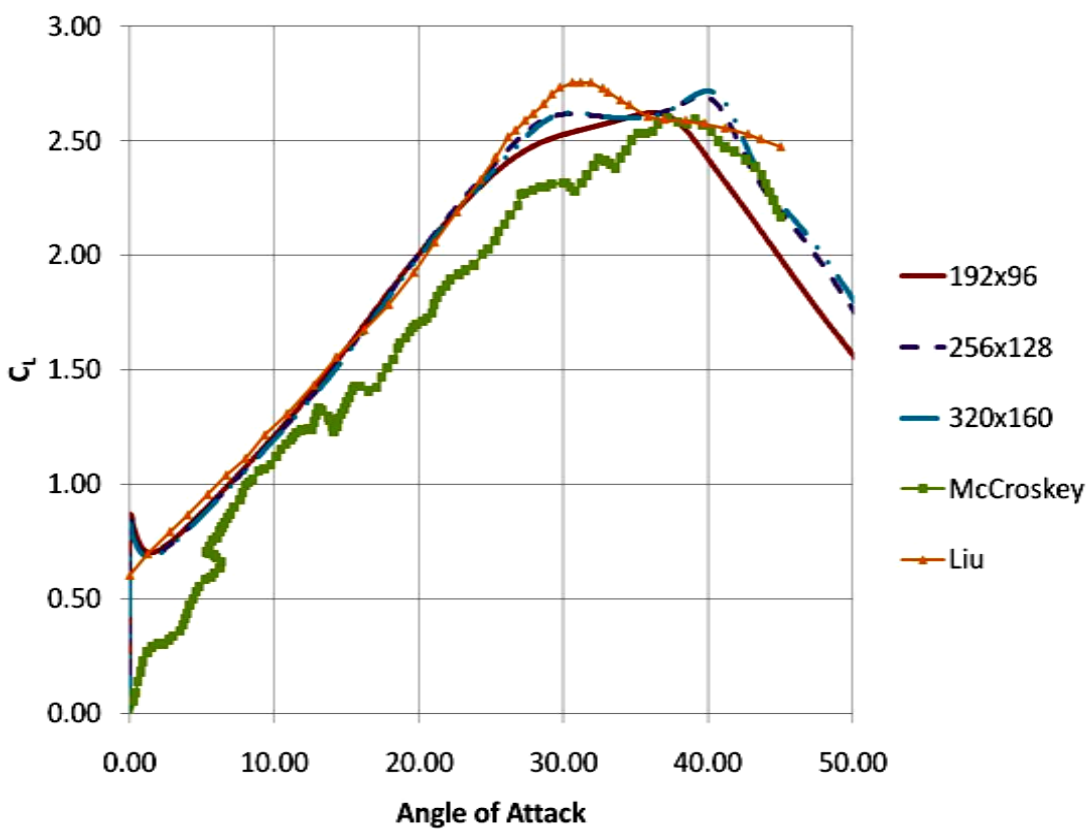

Figure 8. The lift coefficient versus time for three different airfoil grid resolutions on a 300x300 background grid, compared to results from Liu and Kawachi ${ }^{[53]}$ and McCroskey et al. ${ }^{[51]}$. 
Figure 9 compares the vorticity contours for the 192x96, 256x128 and 320x160 airfoil grids on the $300 \times 300$ background grid at $40^{\circ}$ angle of attack, which is the position of the second stall angle in our computation. The vorticity contours show similar convergence compared to the force data in Figure 8. Increasing the grid resolution from $192 \times 96$ to $256 \times 128$ results in a noticeably more well defined leading edge vortex while further refinement results in a negligible difference.
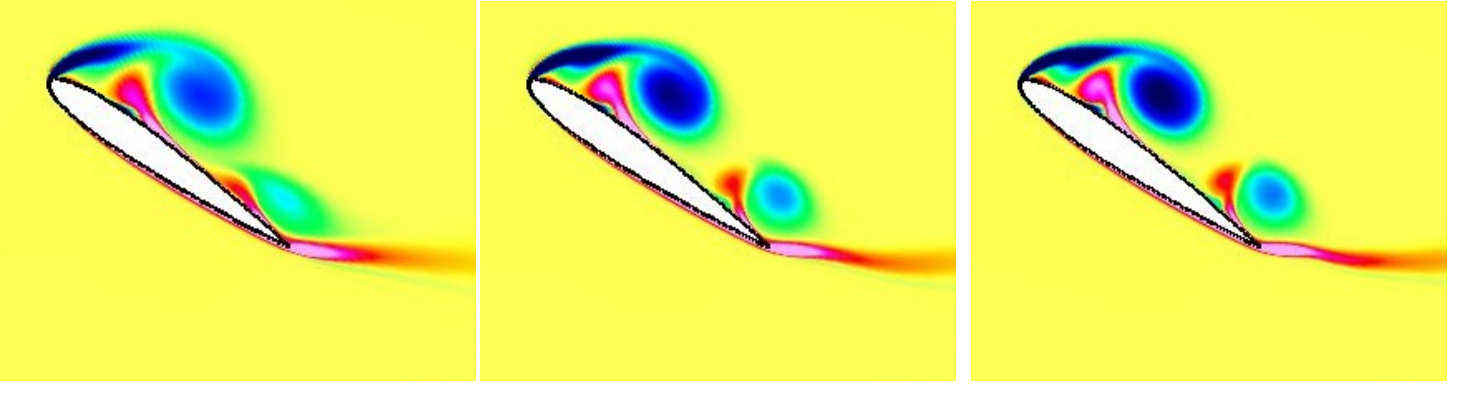

Figure 9. Vorticity contours for $192 \times 96$ (left), 256x128 (middle) and 320x160 (right) airfoil grids on a $300 \times 300$ background grid at $40^{\circ}$ angle of attack. Vorticity contours show more definition for the two finest grids compared to the coarse grid.

\section{E. Grid Sensitivity Analysis}

A grid sensitivity analysis was performed to determine the grid resolution necessary to provide accurate force data as well as to resolve the flow field around the airfoils. The wall boundaries were spaced 20 chord lengths away from the airfoil, which was determined to be sufficient based on the dynamic stall case. The same kinematics were used for the sensitivity analysis as were used for the tandem wing cases, but only a single airfoil was used for the sensitivity analysis. In the study we systematically tested the effect of domain size and grid resolution of each individual overlapping grid, but here we only report the results from different airfoil grid resolutions. Coarse, medium and fine airfoil grid resolutions were tested with $100 \times 50,150 \times 75$ and $200 \times 100$ grid lines respectively in the circumferential and radial directions. A fourth type of grid was also 
tested, with an airfoil grid that had the same grid resolution as the medium grid (O-type grid in Figure 6), but a smaller domain (fewer grid lines in the normal direction) and a finer wake grid resolution. As will become clear, the fourth type of grid is well suited for the tandem wing study.

Figure 10 shows the lift and thrust coefficients over a single flapping cycle for the different grid types.

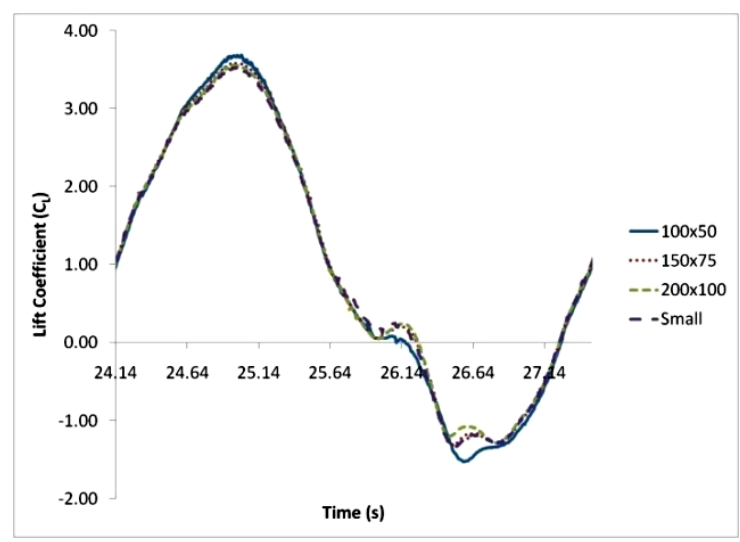

a) Lift

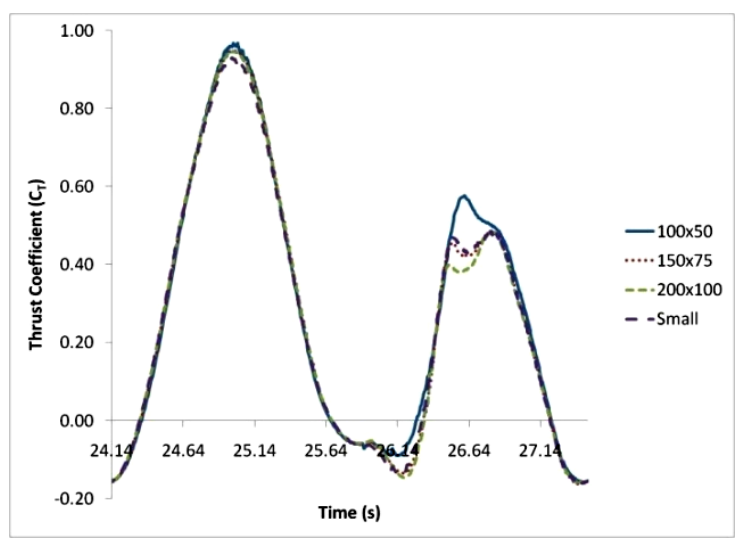

b) Thrust

Figure 10. Lift and thrust data for different grid types used in the sensitivity analysis. a) lift, b)thrust.

Changing the airfoil grid resolution did not have a significant effect on the force data, with less than a $2 \%$ difference between the cycle averaged values for medium and fine grids. There was also very little difference in the force data between the medium grid and the small airfoil grid, however, the wake grid density had a large effect on the flow field. Figure 11 shows that the resulting vorticity contours depended greatly on the different grid type used. Using the medium wake grid (Figure 11a), the vortices dissipate quickly as they move downstream; but the smaller sized airfoil grid with the fine resolution wake grid produced even better results showing well defined vortex structures in the wake (Figure 11b). Since the hindwing performance can be significantly affected 
by the shed vortices from the forewing, it is critical to capture the vortex structure. Based on the sensitivity analysis, it was decided to use the grid type utilizing the smaller sized airfoil grid and a fine resolution wake grid for the tandem wing analysis.

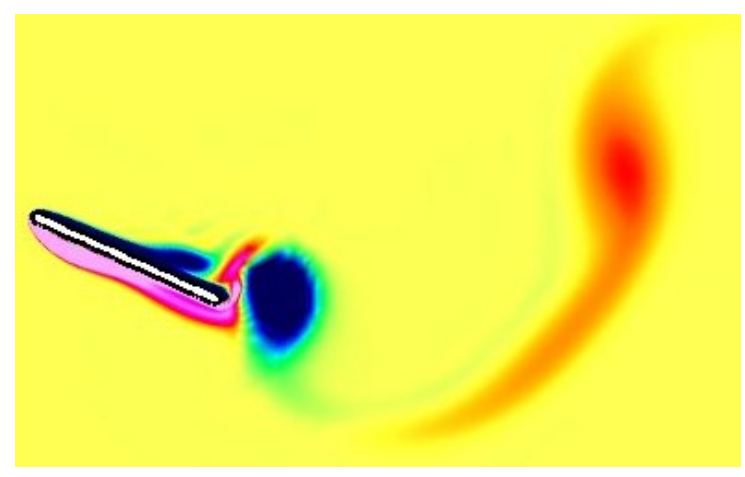

a) Medium Resolution Wake Grid

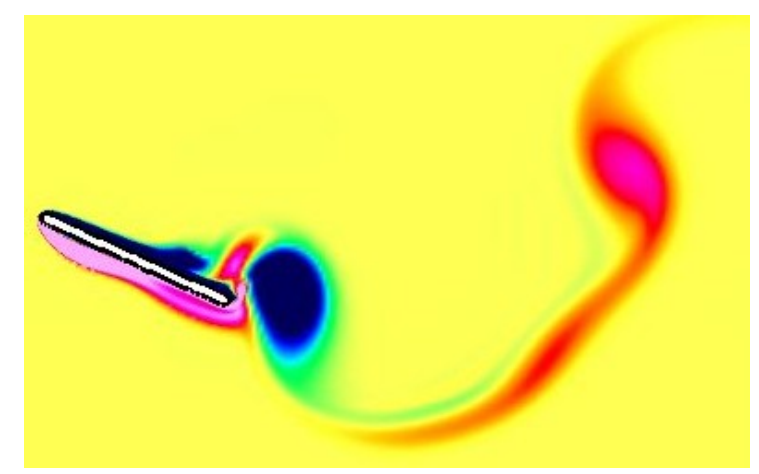

b) Fine Resolution Wake Grid

Figure 11. Vorticity contours for the different grid types used in the sensitivity analysis. A fine wake grid can better resolve the wake flow structure a)medium resolution wake grid, b)fine resolution wake grid and a smaller sized airfoil grid. 


\section{EFFECT OF PHASE ANGLE}

Both experimental and numerical results from previous studies show mixed results when the force coefficients of tandem wings are compared to a single wing (Tandem outperforms single ${ }^{[33,37]}$, single outperforms tandem ${ }^{[26,31]}$ ). Meanwhile, it seems clear that tandem wings can obtain higher efficiency when flapping with the optimal phase angle. In this study, we investigate the phase relationship between tandem flapping wings using a $2 \mathrm{D}$ model. The tandem wings were simulated in forward flight (inlet velocity equal to one chord length per second) at a Reynolds number of $10^{4}$ using an incompressible Navier-Stokes solver. A Reynolds number of $10^{4}$ was chosen, which is at the high end of operation for dragonflies ${ }^{[54]}$. Three different phase relationships were considered, hindwing leading forewing by 0 , 90, and 180 degrees (the phase lag is applied to both the pitching and plunging motions together). To clarify, when the forewing is at the start of the downstroke; at $0^{\circ}$, the hindwing is also at the start of the downstroke; at $90^{\circ}$, the hindwing is halfway through the downstroke; and at $180^{\circ}$, the hindwing is at the start of the upstroke. While three phase angles alone may not be

enough to determine optimization, ${ }^{[55]}$ it should be enough to demonstrate the broad effects that the phase angle has on the aerodynamics. Wing spacing was equal to one chord length for all cases. Detailed comparisons were made with the case of a single airfoil with the same flow conditions, kinematics, and geometry (including chord length). 
The tandem and single wing cases were tested at a Strouhal number of 0.3 , based on the observations by Taylor, et al that most natural fliers flap in a Strouhal number range of 0.2-0.4. ${ }^{[7]}$ Results for all cases were taken after periodic motion had been established which required simulation out to eight flapping cycles.

The objectives of this section are to:

1) Determine the effect of phase angle on the force coefficients and efficiency of the tandem fore and hind wings, individually. Compare these results to a single wing to determine the effect on the hindwing with and without a forewing and vice versa.

2) Determine the effect of the phase angle on the force coefficients and efficiency of the tandem configuration on a systems level (combined fore and hind wing). Compare the tandem wing configuration at each phase angle to a single wing in terms of the lift, thrust, and power coefficients and efficiency in order to determine the effect of the tandem wing interaction on performance. The lift, thrust and power coefficients of the tandem wing are calculated from the combined force and area of the fore and hind wing in order to compare to a single wing.

3) Determine the relationship between the phase angle and wing-wing vortex interactions and how it affects the force generation of the hindwing.

As noted above, comparisons between the tandem wing and single wing were made on two different levels. First, to determine the results of the hindwing with and without a forewing. This is a relatively straightforward comparison, where the single wing results represent the hindwing without the forewing. Second, to compare the performance of the tandem configuration on a system wide level (fore and hind wing 
combined) to the performance of a single wing. Since the force coefficients and efficiency are all normalized for area, the system level results of the tandem wing can be compared directly to a single wing. Since the flow conditions and flapping kinematics are the same between the tandem and single wings, any differences in the force coefficients are due to the tandem wing interaction. That is, if the two tandem wings were isolated, then the force coefficients would be identical to a single wing, therefore any change from the single wing results must be due to the tandem wing interaction.

\section{A. Aerodynamic Forces}

The fore and hind wing results were compared individually to a single wing in order to determine the effect of the forewing on the hindwing (and vice versa). The lift, thrust and resultant coefficients were calculated for each wing individually as

$$
\begin{aligned}
C_{L} & =\frac{L}{0.5 \rho A U^{2}} \\
C_{T} & =\frac{T}{0.5 \rho A U^{2}} \\
C_{R} & =\frac{\sqrt{L^{2}+T^{2}}}{0.5 \rho A U^{2}}
\end{aligned}
$$

where $C_{L}, C_{T}$, and $C_{R}$ are the lift, thrust and resultant force coefficients, $\rho$ is the fluid density, $\mathrm{A}$ is the planform area with unit depth, $\mathrm{U}$ is the freestream velocity and $\mathrm{L}$ and $\mathrm{T}$ are the lift and thrust forces. Figure 12 shows the transient lift and thrust coefficients, over a single flapping cycle, for both the fore and hind wings at all the three tested phase angles, $0^{\circ}, 90^{\circ}$ and $180^{\circ}$ (hindwing leading) as well as the same values for a single wing. All thrust results in this paper are the net thrust, or the total thrust minus the drag. 


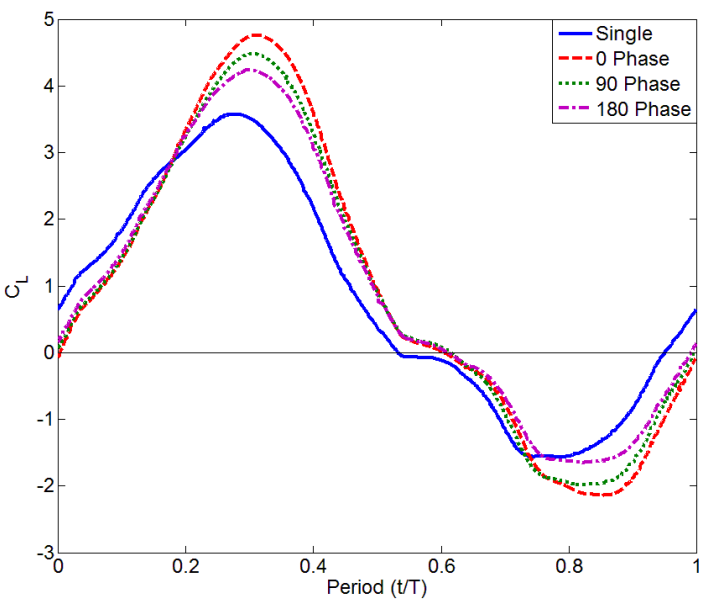

(a)

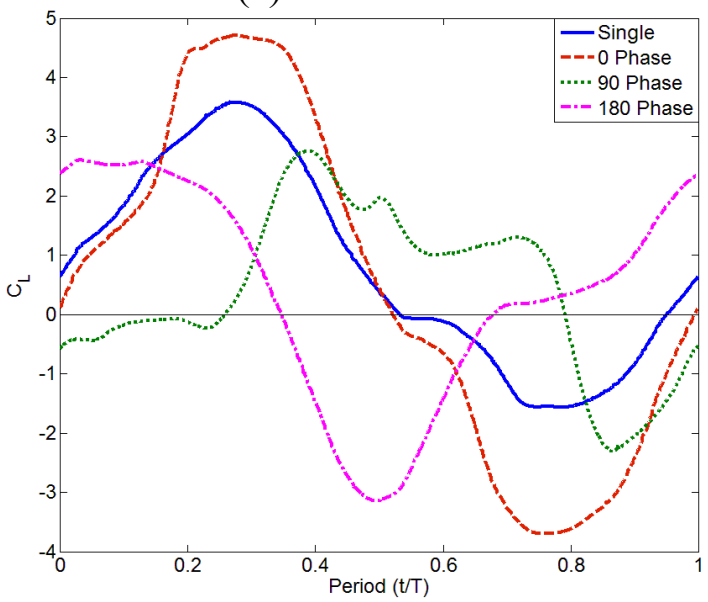

(c)

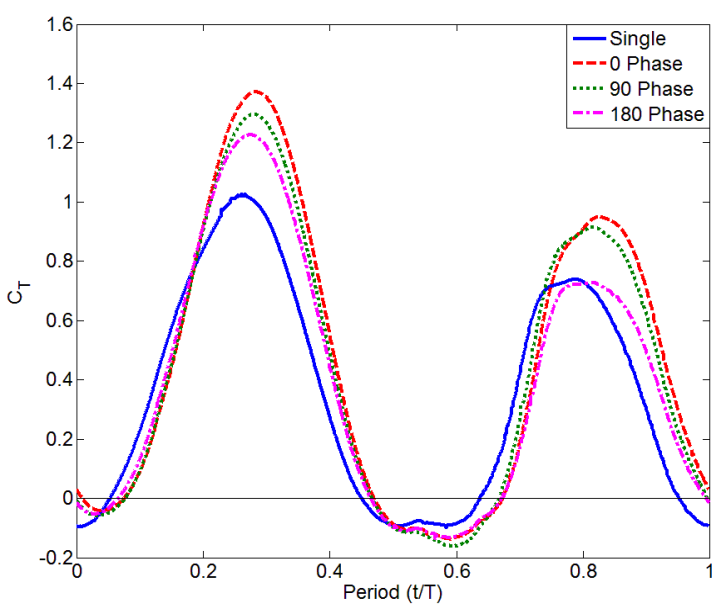

(b)

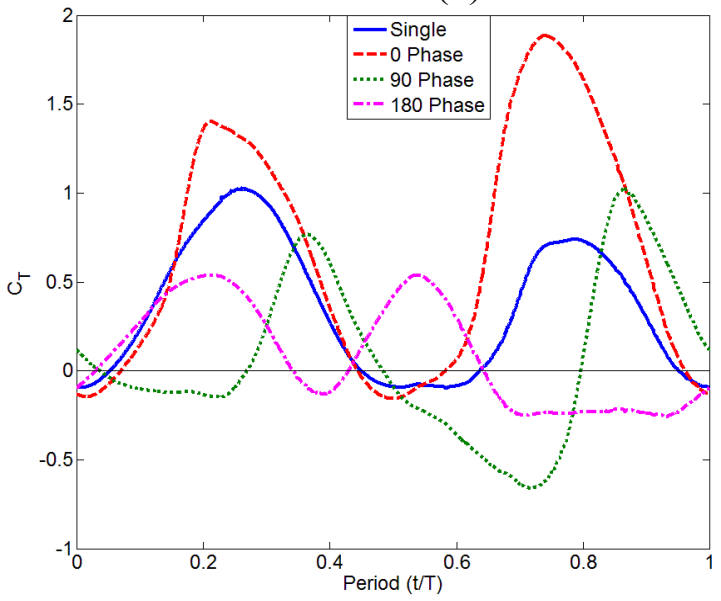

(d)

Figure 12. (a) Lift coefficient during period for single and fore wings. (b) Thrust coefficient during period for single and fore wings. (c) Lift coefficient during period for single and hind wings. (d) Thrust coefficient during period for single and hind wings.

Parts a) and b) show that the presence of the hindwing has a notable effect on the peak lift and thrust coefficients of the forewing during the cycle. The increase in the magnitude of these peaks varies with phase angle, with the $0^{\circ}$ phase case showing the largest increase. This effect is not surprising given the results of Jones et al. that show a pair of oscillating airfoils to the rear of a fixed forewing can cause an entrainment effect on the forewing and increase lift ${ }^{[56]}$. Other than the increased peaks in lift and thrust, the forewing results are fairly similar to a single wing. 
The lift and thrust coefficients for the hindwing, shown in c) and d), vary much more wildly from the single wing and for different phase angles than the forewing. This is very likely due to the change in wing-wing vortex interactions caused by different phase angles, which will be discussed in depth shortly. When the tandem wings flap with $0^{\circ}$ phase lag, the hindwing experiences large increases in the peak lift and thrust coefficients with no noticeable phase lag. Flapping with $90^{\circ}$ and $180^{\circ}$ phase lag; however, causes the hindwing to experience noticeable decreases in the peak lift and thrust coefficients, lower even, than the single wing case. There are also large phase lags in the lift and thrust coefficients of the hind at $90^{\circ}$ and $180^{\circ}$. It is important to note here that the data in c) and d) has been normalized so that at any point along the $\mathrm{x}$-axis, all four cases are at the same position in the flapping cycle (origin is at the top of the downstroke). Notably, this causes the hindwing of the $90^{\circ}$ case to produce all of its positive lift at the second half of the downstroke and first half of the upstroke, while the hindwing of the $180^{\circ}$ case produces most of its positive lift during the second half of the upstroke and first half of the downstroke.

Table 3 summarizes the cycle averaged lift, thrust and resultant force coefficients for the single wing and the fore and hind wings of each tandem case. It also shows the corresponding percentage increase or decrease compared to a single wing. The cycle averaged values were calculated using

$$
\begin{aligned}
& \overline{C_{L}}=\frac{1}{T} \int_{0}^{T} C_{L} d t \\
& \overline{C_{T}}=\frac{1}{T} \int_{0}^{T} C_{T} d t \\
& \overline{C_{R}}=\frac{1}{T} \int_{0}^{T} C_{R} d t
\end{aligned}
$$


where $\overline{C_{L}}$ is the cycle averaged lift coefficient, $\overline{C_{T}}$ is the cycle averaged thrust coefficient, $\overline{C_{R}}$ is cycle averaged resultant coefficient and $\mathrm{T}$ is the period length.

\begin{tabular}{|l|c|c|c|c|c|c|}
\hline \multicolumn{1}{|c|}{ Wing } & $\overline{\mathbf{C}_{\mathbf{L}}}$ & $\overline{\mathbf{C}_{\mathbf{L}}} \mathbf{\%}$ & $\overline{\mathbf{C}_{\mathbf{T}}}$ & $\overline{\mathbf{C}_{\mathbf{T}}} \mathbf{\%}$ & $\overline{\mathbf{C}_{\mathbf{R}}}$ & $\overline{\mathbf{C}_{\mathbf{R}}} \mathbf{\%}$ \\
\hline Single & 0.807 & - & 0.350 & - & 0.879 & - \\
\hline $0^{\circ}$ Phase Fore & 0.924 & 14.5 & 0.459 & 31.4 & 1.032 & 17.4 \\
\hline $90^{\circ}$ Phase Fore & 0.890 & 10.4 & 0.433 & 24.0 & 0.990 & 12.6 \\
\hline $180^{\circ}$ Phase Fore & 0.941 & 16.7 & 0.389 & 11.3 & 1.018 & 15.9 \\
\hline $0^{\circ}$ Phase Hind & 0.429 & -46.8 & 0.660 & 88.8 & 0.787 & -10.5 \\
\hline $90^{\circ}$ Phase Hind & 0.398 & -50.6 & 0.070 & -79.9 & 0.404 & -54.0 \\
\hline $180^{\circ}$ Phase Hind & 0.378 & -53.1 & 0.088 & -74.9 & 0.388 & -55.9 \\
\hline
\end{tabular}

Table 3. Summary of lift, thrust and resultant for single, fore and hind wings.

From Table 3 it is clear that the presence of the hindwing has a positive effect on the force coefficients of the forewing. Compared to a single wing, without a hindwing, the forewing shows increases in the average lift, thrust and resultant coefficients. The phase angles tested only show relatively small variations in the lift and resultant coefficients, but large variation in the thrust coefficient, with $0^{\circ}$ phase showing the largest increase in the thrust coefficient and $180^{\circ}$ phase showing the smallest increase in the thrust coefficient.

The presence of the forewing had very mixed results on the hindwing, when compared to a single wing without a forewing. The hindwing showed a decrease in the lift coefficient of about $50 \%$, regardless of phase angle while the thrust coefficient was increased by about $90 \%$ for the $0^{\circ}$ phase case and decreased by almost $80 \%$ for both the $90^{\circ}$ and $180^{\circ}$ phase cases. The resultant coefficient of the hindwing was reduced at all three phase angles, with only a $10 \%$ reduction at $0^{\circ}$ and just over a $50 \%$ reduction at $90^{\circ}$ and $180^{\circ}$.

The power required to actuate the wing was calculated using 


$$
C_{p}=\frac{1}{0.5 \rho A U^{3}} \int_{0}^{T}[-(L \cdot V)-(M \cdot \omega)] d t
$$

where $C_{p}$ is the power coefficient, $\rho$ is the fluid density, $A$ is the planform area with unit depth of an individual wing, $\mathrm{U}$ is the flow velocity, $\mathrm{T}$ is the flapping period, $\mathrm{L}$ is the instantaneous lift force, $\mathrm{V}$ is the instantaneous wing vertical velocity, $\mathrm{M}$ is the instantaneous pitching moment, and $\omega$ is the instantaneous rotational velocity. $C_{p}$ is defined such that a positive $C_{p}$ represents power output by the system and negative $C_{p}$ is power put back into the system. Since it is impractical for the wing to regenerate power during the flapping cycle, the calculation of $\mathrm{C}_{\mathrm{p}}$ was modified such that when the one of the terms in the integrand was negative $(-L \cdot V$ or $-M \cdot \omega)$ the negative term was changed to zero for the integration. So when the force opposes the motion, positive actuation power is required, and when the force is coincident with the motion, zero actuation power is required (rather than negative actuation power). Further references to $C_{p}$ refer to this modified $\mathrm{C}_{\mathrm{p}}$. In the cases studied, the power contributed by the moment term was negligible compared to the lift term.

Table 4 shows the power coefficient as well as the propulsive, lift and resultant efficiencies for each wing at each phase angle. The propulsive, lift and resultant efficiencies, were calculated using

$$
\begin{gathered}
\eta_{P}=\frac{\overline{C_{T}}}{\overline{C_{P}}} \\
\eta_{L}=\frac{\overline{C_{L}}}{C_{P}} \\
\eta_{R}=\frac{\overline{C_{R}}}{C_{P}}
\end{gathered}
$$


where $\eta_{\mathrm{P}}$ is the propulsive efficiency, $\overline{C_{T}}$ is the cycle averaged thrust coefficient, $\mathrm{C}_{\mathrm{P}}$ is the power coefficient, $\eta_{\mathrm{L}}$ is the lift efficiency, $\overline{C_{L}}$ is the cycle averaged lift coefficient, $\eta_{\mathrm{R}}$ is the resultant efficiency and $\overline{C_{R}}$ is the cycle averaged resultant coefficient.

\begin{tabular}{|l|c|c|c|c|}
\hline \multicolumn{1}{|c|}{ Wing } & $\mathbf{C}_{\mathbf{P}}$ & $\boldsymbol{\eta}_{\mathbf{P}}$ & $\boldsymbol{\eta}_{\mathbf{L}}$ & $\boldsymbol{\eta}_{\mathbf{R}}$ \\
\hline Single & 1.137 & $30.8 \%$ & $71.0 \%$ & $77.3 \%$ \\
\hline $0^{\circ}$ Phase Fore & 1.500 & $30.6 \%$ & $61.6 \%$ & $68.8 \%$ \\
\hline $90^{\circ}$ Phase Fore & 1.422 & $30.5 \%$ & $62.6 \%$ & $69.6 \%$ \\
\hline $180^{\circ}$ Phase Fore & 1.268 & $30.7 \%$ & $74.2 \%$ & $80.3 \%$ \\
\hline $0^{\circ}$ Phase Hind & 1.920 & $34.4 \%$ & $22.3 \%$ & $41.0 \%$ \\
\hline $90^{\circ}$ Phase Hind & 0.410 & $17.1 \%$ & $97.1 \%$ & $98.5 \%$ \\
\hline $180^{\circ}$ Phase Hind & 0.488 & $18.0 \%$ & $77.5 \%$ & $79.5 \%$ \\
\hline
\end{tabular}

Table 4. Power coefficient and lift, propulsive and resultant efficiencies for the single wing and the fore and hind wing at each phase angle.

The results in Table 4 are quite interesting. First, when operating at a $90^{\circ}$ and $180^{\circ}$ phase lag, the hindwing requires very little power for actuation. Despite undergoing the exact same flapping kinematics, the hindwing only requires $36 \%$ and $43 \%$ of the power needed to actuate the single wing, when operating at $90^{\circ}$ and $180^{\circ}$ phase angles, respectively. This clearly shows that the hindwing is able to extract power from the wake of the forewing at certain phase angles. The large decrease in the actuation power was a result of the phase shift in the lift shown in Figure 12c. Both the $90^{\circ}$ and $180^{\circ}$ phase cases produce a large amount of positive lift during the upstroke, which corresponds to the direction of motion of the wing and reduces the power needed for actuation.

The hindwing has no noticeable effect on the propulsive efficiency of the forewing, regardless of phase angle. Its effect on the forewing's lift efficiency is more noticeable, decreasing it by about $12 \%$ at phase angles of $0^{\circ}$ and $90^{\circ}$ and increasing it slightly at $180^{\circ}$. For the resultant efficiency, the forewing for the $0^{\circ}$ and $90^{\circ}$ cases showed about a $12 \%$ drop in efficiency, compared to the single wing, while the forewing for the $180^{\circ}$ case showed a slight increase in the resultant efficiency. 
The effect of the forewing on the hindwing shows mixed results for efficiency. At $0^{\circ}$ phase, the hindwing showed a small increase in propulsive efficiency over a single wing, but at $90^{\circ}$ and $180^{\circ}$ it only had about half the propulsive efficiency of a single wing. Conversely, the hindwing flapping at $0^{\circ}$ phase lag only had approximately one third the lift efficiency of the single wing, while the $90^{\circ}$ and $180^{\circ}$ hindwings showed an increase in the lift efficiency of $37 \%$ and $9 \%$ respectively. The resultant efficiency of the hindwing at $0^{\circ}$ phase lag was about half of the resultant efficiency of a single wing while the $90^{\circ}$ phase hindwing showed an increase of $30 \%$ and the $180^{\circ}$ phase hindwing showed a slight increase in the resultant efficiency.

Next, the performance of the tandem configuration was compared on a systems level (combined fore and hind wing) to a single wing. For this comparison, the cycle averaged lift, thrust and resultant coefficients were calculated using

$$
\begin{gathered}
\overline{C_{L}}=\frac{L_{F}+L_{H}}{0.5 \rho\left(A_{F}+A_{H}\right) U^{2}} \\
\overline{C_{T}}=\frac{T_{F}+T_{H}}{0.5 \rho\left(A_{F}+A_{H}\right) U^{2}} \\
\overline{C_{R}}=\frac{\sqrt{\left(L_{F}+L_{H}\right)^{2}+\left(T_{F}+T_{H}\right)^{2}}}{0.5 \rho\left(A_{F}+A_{H}\right) U^{2}}
\end{gathered}
$$

where $L_{F}$ and $L_{H}$ are the cycle averaged lift of the fore and hind wing, $T_{F}$ and $T_{H}$ are the cycle averaged thrust of the fore and hind wing, and $\mathrm{A}_{\mathrm{F}}$ and $\mathrm{A}_{\mathrm{H}}$ are the planform area of the fore and hind wing with unit depth. Since the combined force production of the tandem configuration is normalized by the planform area of both wings, the lift, thrust and resultant coefficients can be compared to the same results for a single wing. 
Table 5 shows the results for cycle average lift, thrust and resultant coefficients for each tandem configuration (fore and hind wings combined) compared to the results of a single wing.

\begin{tabular}{|l|c|c|c|c|c|c|}
\hline \multicolumn{1}{|c|}{ Wing } & $\overline{\mathbf{C}_{\mathbf{L}}}$ & $\overline{\mathbf{C}_{\mathbf{L}}} \mathbf{\%}$ & $\overline{\mathbf{C}_{\mathbf{T}}}$ & $\overline{\mathbf{C}_{\mathbf{T}}} \mathbf{\%}$ & $\overline{\mathbf{C}_{\mathbf{R}}}$ & $\overline{\mathbf{C}_{\mathbf{R}}}$ \% \\
\hline Single & 0.807 & - & 0.350 & - & 0.879 & - \\
\hline $0^{\circ}$ Phase & 0.677 & -16.1 & 0.560 & 60.1 & 0.878 & -0.11 \\
\hline $90^{\circ}$ Phase & 0.644 & -20.1 & 0.252 & -28.0 & 0.691 & -21.3 \\
\hline $180^{\circ}$ Phase & 0.660 & -18.2 & 0.239 & -31.8 & 0.701 & -20.2 \\
\hline
\end{tabular}

Table 5. Comparison of lift, thrust and resultant for two isolated single wings to each tandem configuration.

The data from Table 5 shows that the magnitude of the resultant for the $0^{\circ}$ phase case is similar to that of the single wing, while both the $90^{\circ}$ and $180^{\circ}$ phase cases each exhibit about the same decrease in the resultant, about $20 \%$. The total lift and thrust of the $90^{\circ}$ and $180^{\circ}$ phase cases is less than the case of the single wing. The $0^{\circ}$ phase case generates $16 \%$ less total lift than the single wing, but $60 \%$ more total thrust. Overall, the $90^{\circ}$ and $180^{\circ}$ phase cases are detrimental in terms of lift, thrust and the resultant compared to the single wing. The $0^{\circ}$ phase case causes no change to the magnitude of the resultant; however, it inclines the resultant forward, producing more thrust at the expense of lift.

Table 6 shows the power coefficient and propulsive, lift and resultant efficiencies of the tandem wing configuration (fore and hind wing combined) compared to a single wing.

\begin{tabular}{|l|c|c|c|c|}
\hline Configuration & $\mathbf{C}_{\mathbf{P}}$ & $\boldsymbol{\eta}_{\mathbf{P}}$ & $\boldsymbol{\eta}_{\mathbf{L}}$ & $\boldsymbol{\eta}_{\mathbf{R}}$ \\
\hline Single & 1.137 & $30.8 \%$ & $71.0 \%$ & $77.3 \%$ \\
\hline $0^{\circ}$ Phase & 1.710 & $32.7 \%$ & $39.6 \%$ & $51.3 \%$ \\
\hline $90^{\circ}$ Phase & 0.916 & $27.5 \%$ & $70.3 \%$ & $75.5 \%$ \\
\hline $180^{\circ}$ Phase & 0.878 & $27.2 \%$ & $75.1 \%$ & $79.9 \%$ \\
\hline
\end{tabular}

Table 6. Power coefficient and lift, propulsive and resultant efficiencies for the single wing and the tandem configuration at each phase angle. 
The $0^{\circ}$ phase configuration has the highest power coefficient, significantly higher than the single wing value. Switching to either $90^{\circ}$ or $180^{\circ}$ phase cuts the power coefficient to approximately $50 \%$ of the $0^{\circ}$ phase case, well below the power coefficient of the single wing. The $0^{\circ}$ phase configuration has a slightly higher propulsive efficiency than the single wing, while both the $90^{\circ}$ and $180^{\circ}$ phase configurations have a slightly lower propulsive efficiency than a single wing. The $0^{\circ}$ phase case, however, has a significantly lower lift and resultant efficiency than a single wing, while the $90^{\circ}$ and $180^{\circ}$ have very similar lift and resultant efficiencies as a single wing.

Summarizing the effects of the phase angle on the tandem wing configuration, it is clear that by switching the phase angle, the tandem wing is able to change its flight mode. When flapping with $0^{\circ}$ phase lag, the tandem wing produces a large amount of thrust with a high propulsive efficiency but requires more power for actuation, which lowers the lift and resultant efficiencies. Switching to $90^{\circ}$ or $180^{\circ}$ phase lags lowers the required power for actuation by $50 \%$ and raises the lift and resultant efficiency, but at the expense of thrust and propulsive efficiency. Lift production was nearly identical between the three tested phase angles. These results seem to line up with the observed behavior of dragonflies, where they flap inphase for maneuvers and out of phase for cruising flight.

For the parameters studied in this paper, the tandem wing does not definitively outperform the single wing. While flapping with $0^{\circ}$ phase lag results in a larger thrust coefficient and a higher propulsive efficiency than a single wing, there is a noticeable reduction in lift and increase in the power coefficient which results in a large decrease in the lift and resultant efficiencies. Switching to a phase lag of $90^{\circ}$ or $180^{\circ}$ results in similar lift and resultant efficiencies as a single wing as well as a smaller power 
coefficient, but the single wing still has the larger lift coefficient as well as a larger thrust coefficient and greater propulsive efficiency.

\section{B. Flow Visualization Results}

Figure 13 shows the vorticity contours around the single wing and the hindwings of the three tandem configurations at four different points in the flapping cycle $(0 \%, 25 \%$, $50 \%, 75 \%)$. Red represents counterclockwise (CCW) vorticity and blue represents clockwise $(\mathrm{CW})$ vorticity. Figure 13 is arranged such that each row shows the four different cases at the same point in the flapping cycle. This is specifically highlights how the change in the phase angle affects the vortex generation of the hindwing.

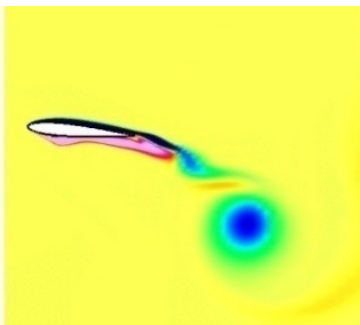

a)

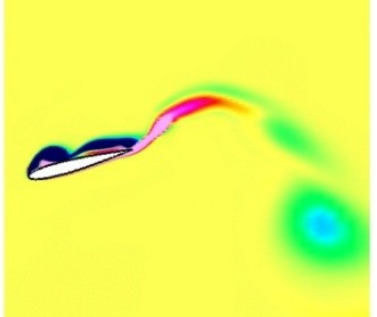

e)

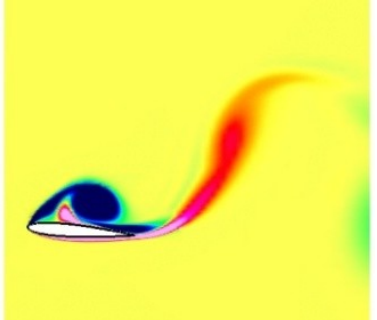

i)

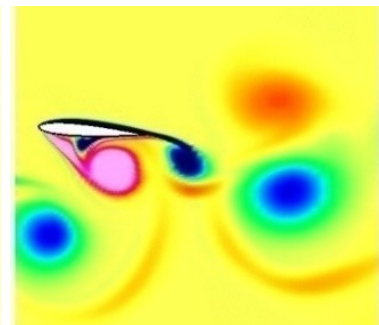

b)

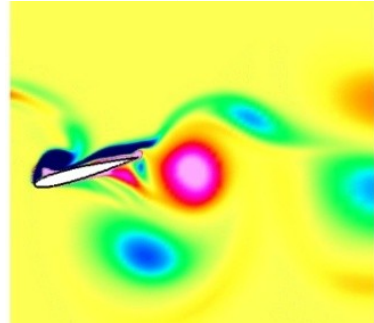

f)

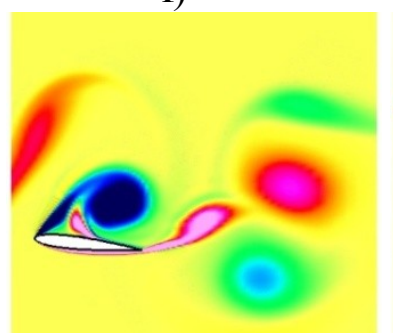

j)

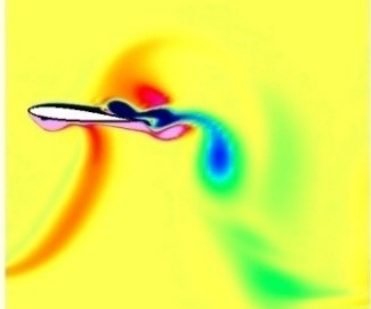

c)

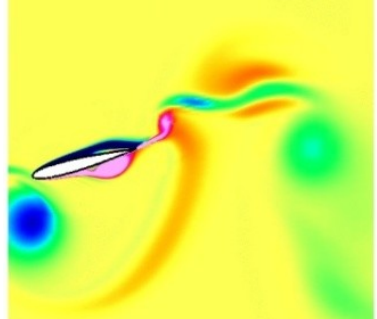

g)

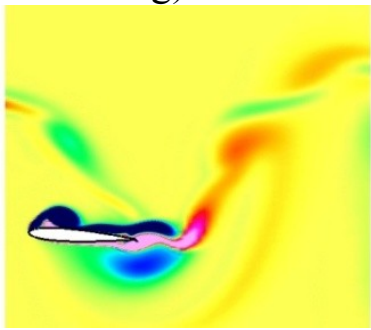

k)

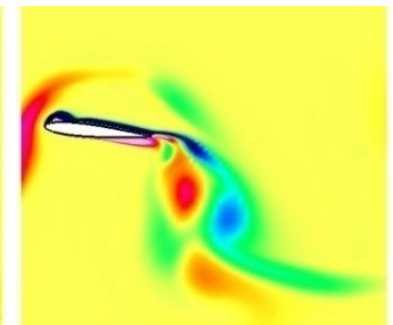

d)

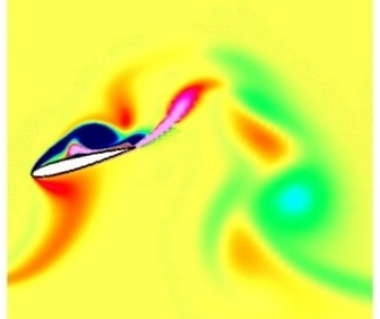

h)

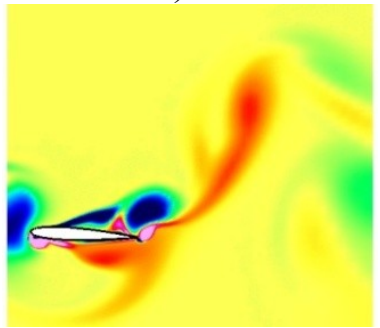

1) 


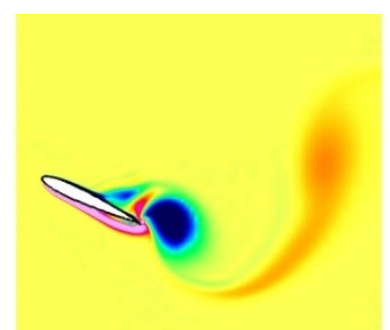

m)

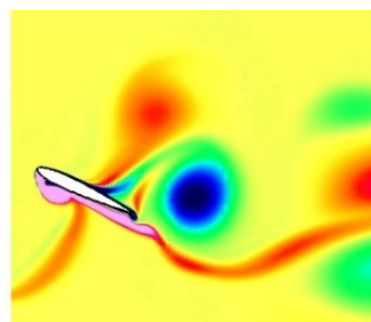

n)

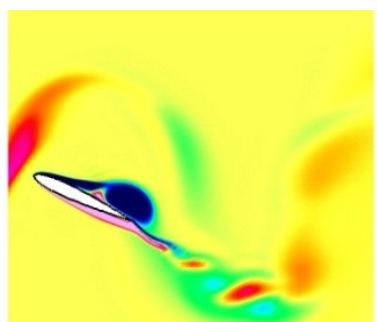

o)

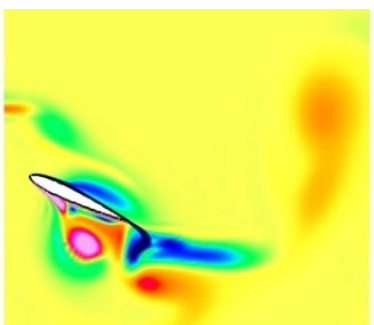

p)

Figure 13. Different phase angles change the vortex shedding between the fore and hind wing, which affects the vorticity around the hindwing. The first row shows the four different cases at $0 \%$ flapping cycle $(a-d)$, the second row $-25 \%(e-h)$, the third row $-50 \%(i-l)$ and the fourth row $-75 \%(m-p)$. The first column is the single wing, the second column is the $0^{\circ}$ hindwing, the third column is the $90^{\circ}$ hindwing and the fourth column is the $180^{\circ}$ hindwing.

Figure 13 shows that the phase angle has a noticeable effect on the size of the leading edge vortex (LEV) generated by the hindwing. Comparing the different tandem configurations, the $0^{\circ}$ phase hindwing was characterized by constructive vortex interactions, while the $90^{\circ}$ and $180^{\circ}$ phase cases were characterized by destructive vortex interactions.

For the $0^{\circ}$ phase case, constructive interactions with the vortices shed by the forewing led to increased size of the LEV's generated around the hindwing, which is seen clearly in $b$ ), f), j), and n). During the first half of the downstroke (b to f), the hindwing passes behind $\mathrm{CW}$ vorticity shed by the forewing, which interacts with the CW LEV generated on the top of the hindwing. A similar interaction occurs during the first half of the downstroke $(\mathrm{j}-\mathrm{n})$, where the hindwing passes behind CCW vorticity shed by the forewing, which interacts with the CCW LEV generated on the bottom of the hindwing. This interaction is more noticeable in Figure 14, which shows the vorticity contours of the fore and hind wing together.

For the $90^{\circ}$ phase hindwing, the destructive interaction caused the lack of an LEV on the top of the airfoil during the downstroke, but the generation of an LEV on the top 
of the airfoil during the upstroke. The first of these destructive interactions occurs as the hindwing reaches the end of the upstroke and starts on the downstroke $(\mathrm{o}-\mathrm{c})$. The hindwing passes behind CCW vorticity shed by the forewing which dampens out the CW LEV that would normally form on the top of the airfoil during the downstroke, while initiating the generation of a CCW LEV on the bottom of the hindwing. A similar interaction occurs as the hindwing moves from the end of the downstroke to the beginning of the upstroke $(\mathrm{g}-\mathrm{k})$ and passes behind a $\mathrm{CW}$ vortex shed by the forewing. This creates a CW LEV that stays attached to the top of the hindwing during part of the upstroke while canceling out the CCW LEV that would normally form on the bottom of the airfoil.

The $180^{\circ}$ phase hindwing experienced similar destructive interactions as the $90^{\circ}$ phase hindwing, but to a lesser extent. At the start of the downstroke (d), the hindwing has already formed a small CW LEV on top, which is earlier than normal. As it starts on the downstroke (d), the hindwing passes behind CCW vorticity shed by the forewing as the CW LEV is forming on the top of the airfoil. This interaction reduces the size of the LEV formed during the downstroke. As the hindwing starts its upstroke (1), a CW vortex shed from the forewing passes over it. Half of this vortex convects over the top of the hindwing, while the other half interacts with the CCW LEV forming at the bottom of the airfoil and detaches it from the hindwing during the upstroke (p).

Figure 14-Figure 16 compare how the changes in the LEV production of the hindwing due to different phase angles affects the lift and thrust production of the hindwing. Each figure depicts results for the $0^{\circ}, 90^{\circ}$ and $180^{\circ}$ tandem cases, respectively. The transient lift and thrust coefficients of the hindwing are plotted to the left along with 
the single wing as a baseline. Vorticity contours are shown to the right at four different points in the flapping cycle, $0 \%, 25 \%, 50 \%$ and $75 \%$ (the same points as in Figure 13). These points in the flapping cycle are marked on the lift and thrust graphs as a), b), c) and d).
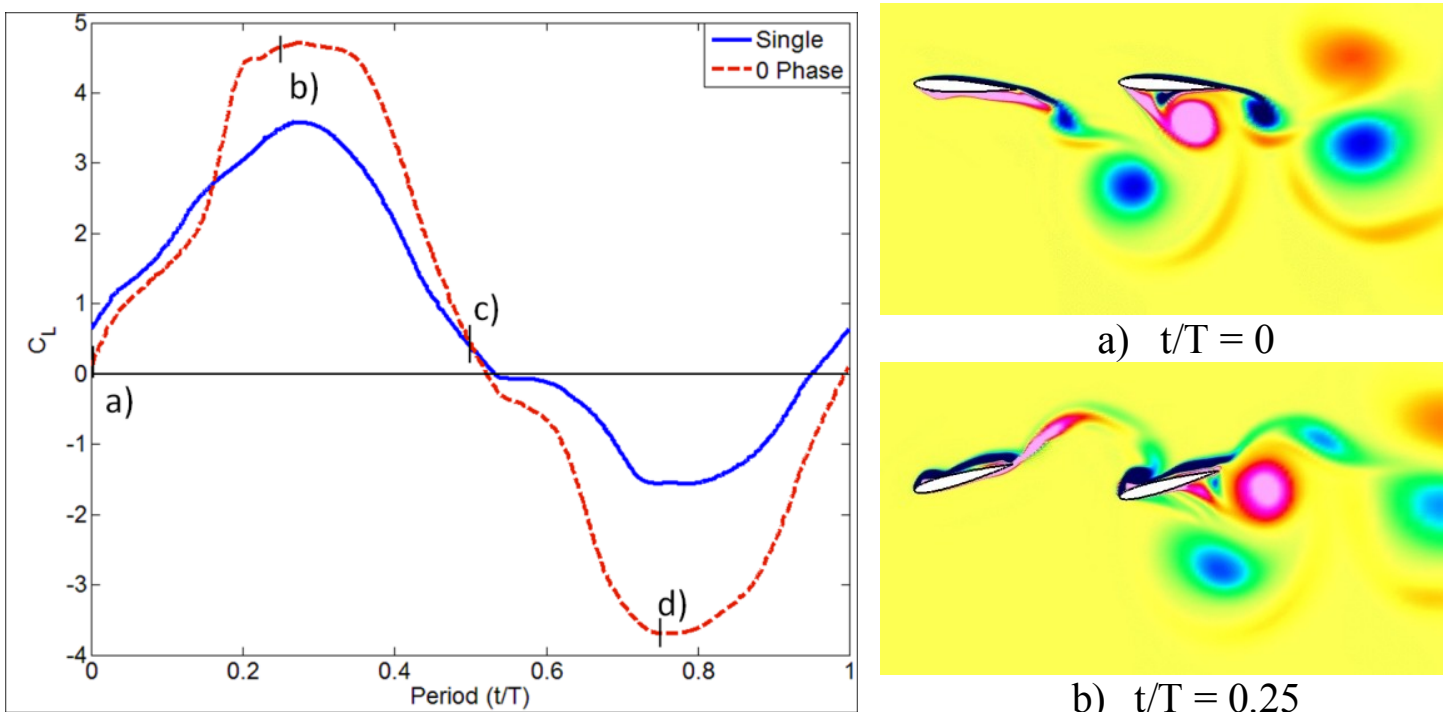

a) $t / T=0$

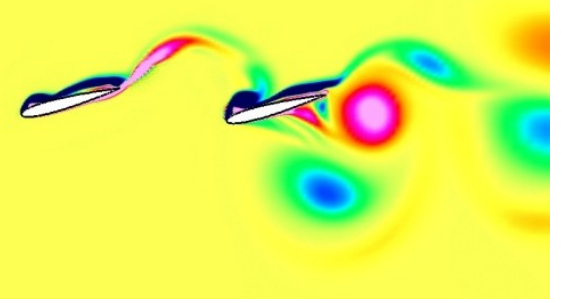

b) $\mathrm{t} / \mathrm{T}=0.25$
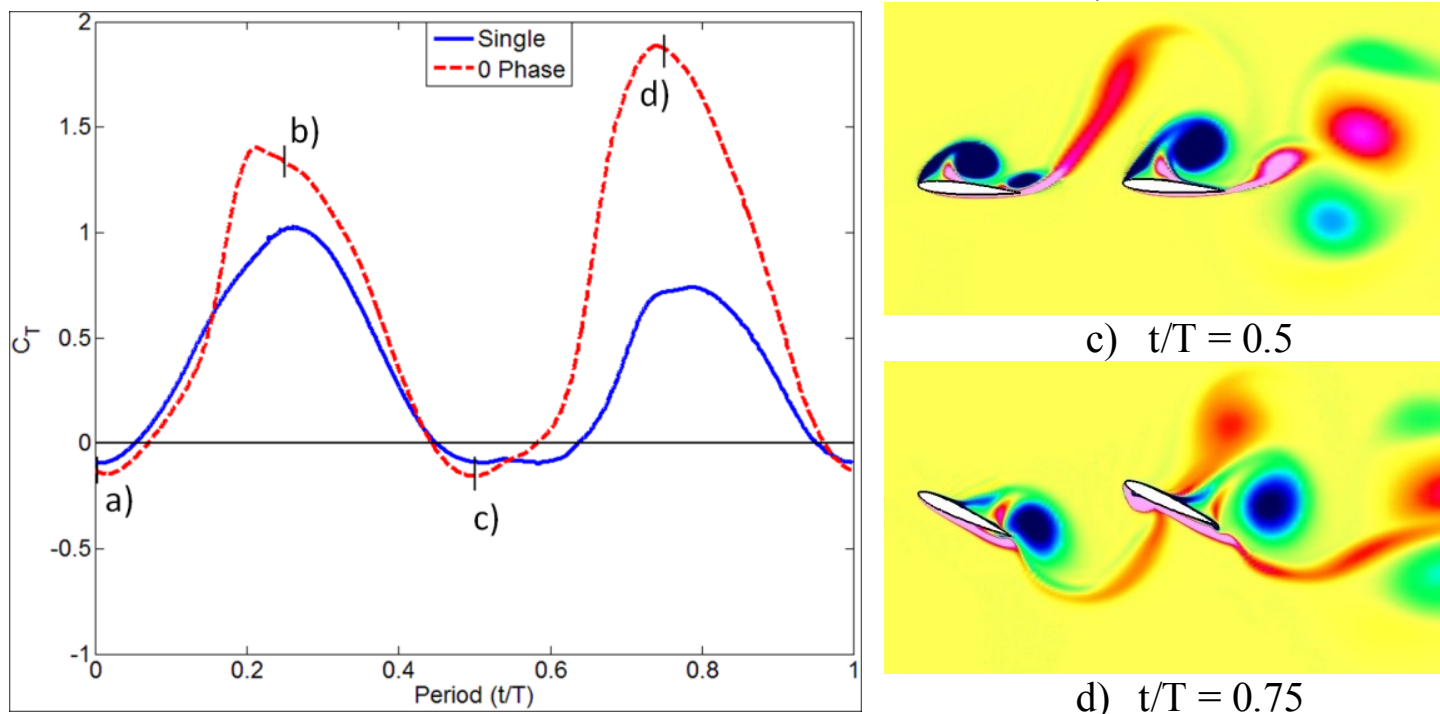

c) $\mathrm{t} / \mathrm{T}=0.5$

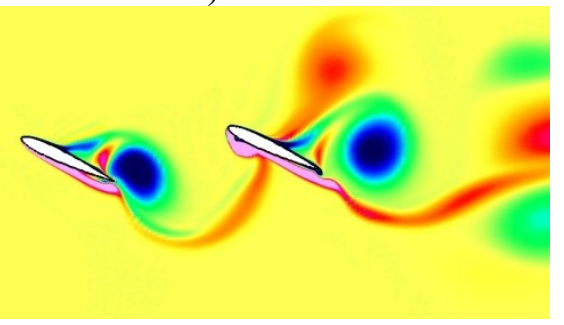

d) $\mathrm{t} / \mathrm{T}=0.75$

Figure 14. Lift and thrust coefficients plotted vs. time and vorticity contours at $0 \%$, $25 \%, 50 \%$ and $75 \%$ flapping cycle for the tandem wing flapping with $0^{\circ}$ phase lag. Constructive vortex interactions increase the size of the LEVs generated by the hindwing which increases the peak lift and thrust production.

Figure 14 shows the lift and thrust coefficients as well as the vorticity contours for the tandem wing flapping with $0^{\circ}$ phase lag. Due to the constructive vortex interaction 
between the fore and hind wing, the hindwing generates larger LEVs on both the downstroke and upstroke. During the downstroke, the LEV is generated on the top of the airfoil. With the airfoil pitching downward, the LEV is also on the upstream side of the airfoil. This point corresponds to b) on the force histories, which is the point of peak lift and thrust production on the downstroke. The same effect is seen on the upstroke. An LEV forms on the bottom of the airfoil, which, with the airfoil pitching upward, is also on the upstream side. This is point d) on the force histories, and it corresponds to peak lift and thrust production on the upstroke. 

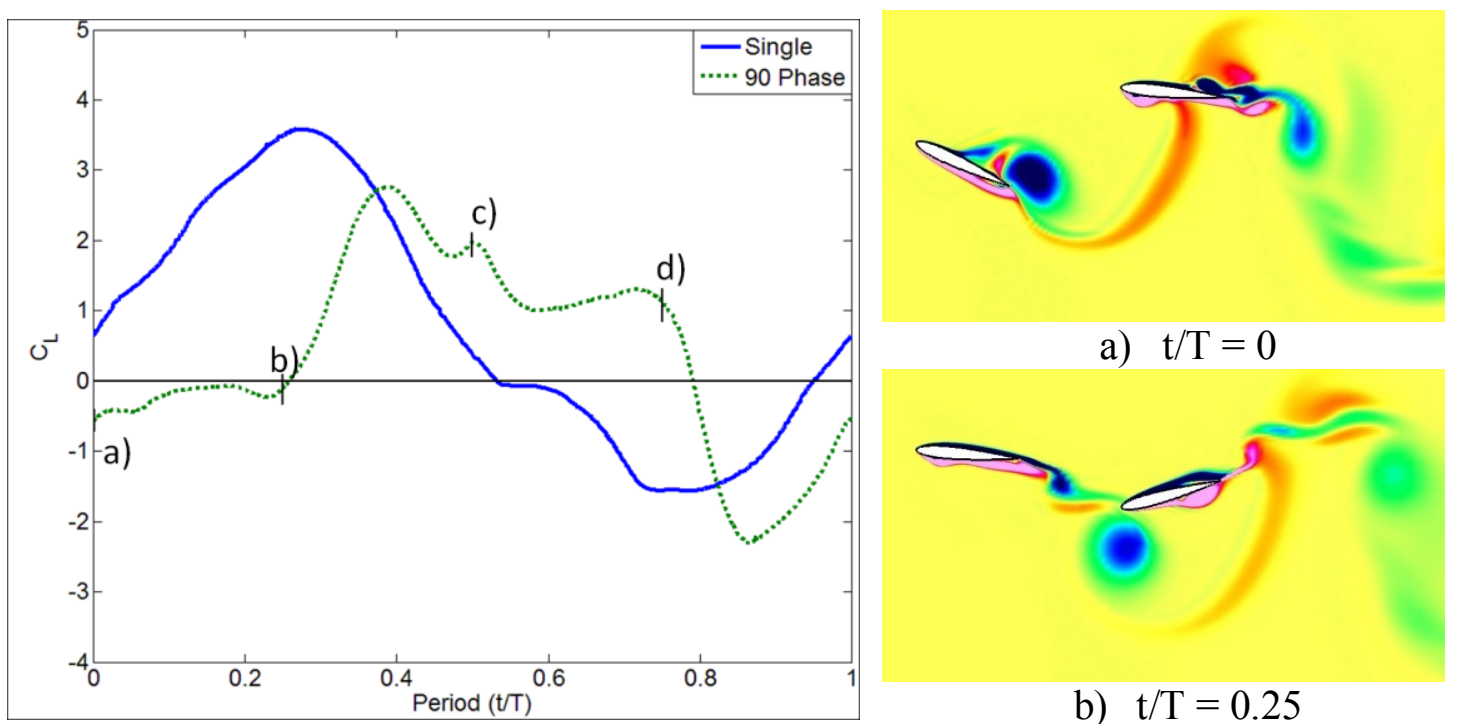

a) $t / T=0$

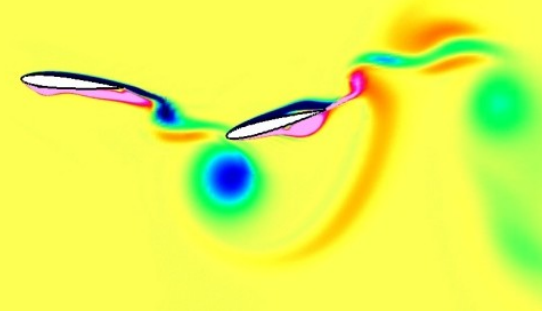

b) $\mathrm{t} / \mathrm{T}=0.25$
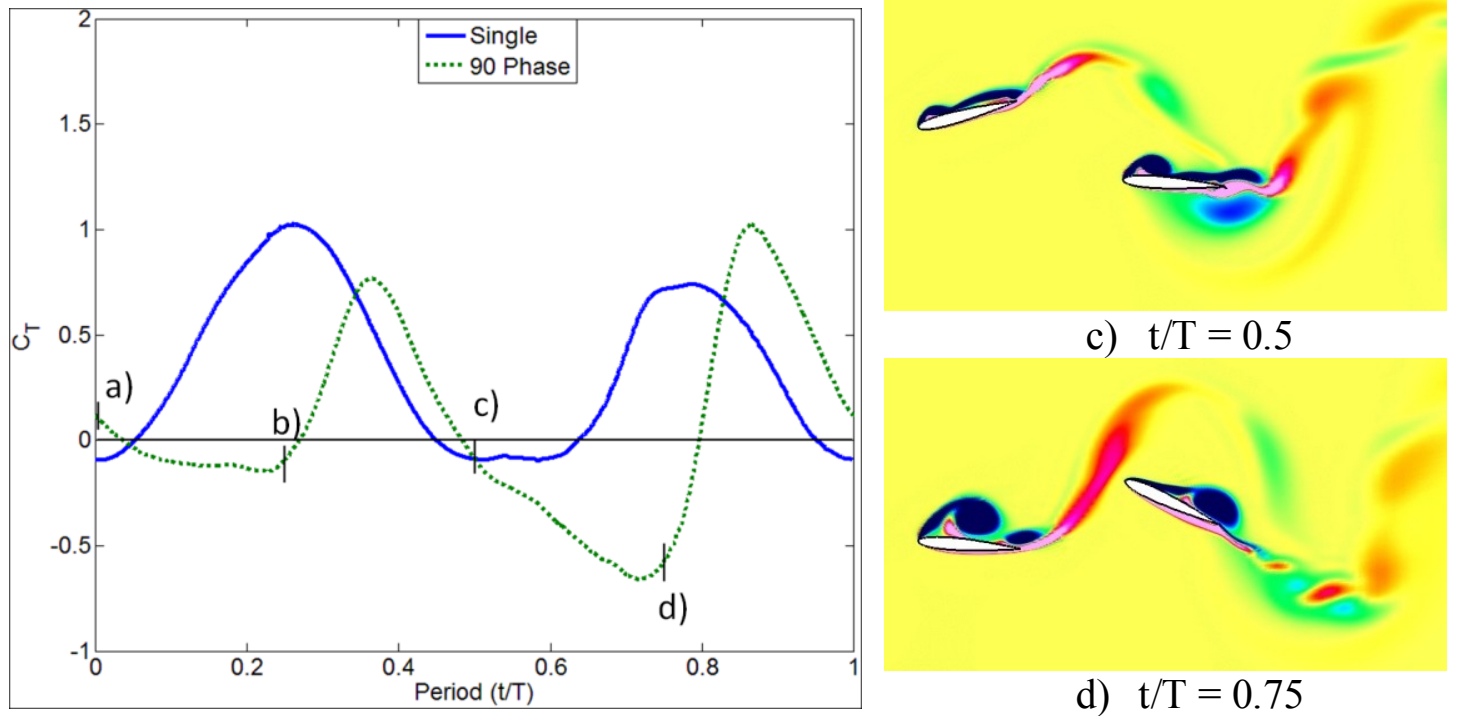

c) $\mathrm{t} / \mathrm{T}=0.5$

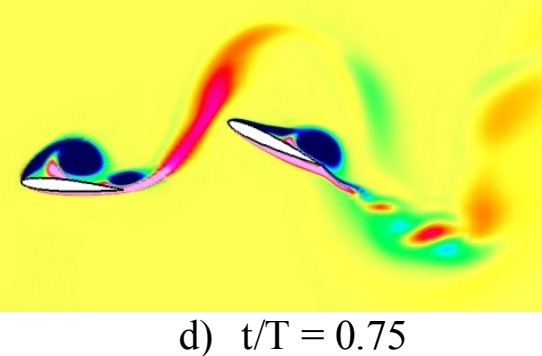

Figure 15. Lift and thrust coefficients plotted vs. time and vorticity contours at $0 \%$, $25 \%, 50 \%$ and $75 \%$ flapping cycle for the tandem wing flapping with $90 \circ$ phase lag. Destructive vortex interactions decrease the size of the LEVs generated by the hindwing and cause a phase shift in the lift such that lift is produced on the upstroke.

Figure 15 shows the results for the tandem wing flapping with $90^{\circ}$ phase lag.

Destructive vortex interactions result in the lack of an LEV on the top of the hindwing during the downstroke. Instead, an LEV is formed on the top of the hindwing during the upstroke. This corresponds to points c) and d) on the force graphs. This causes positive lift to be generated during the upstroke, as well as the large amount of negative thrust, 
point d). This negative thrust corresponds with the LEV on top of the airfoil also being oriented on the downstream side of the airfoil, since the airfoil is pitching upward at this point. Because the hindwing produces a large amount of lift on the upstroke, the power required for actuation is greatly lessened, since the lift production corresponds to the direction of travel of the airfoil.
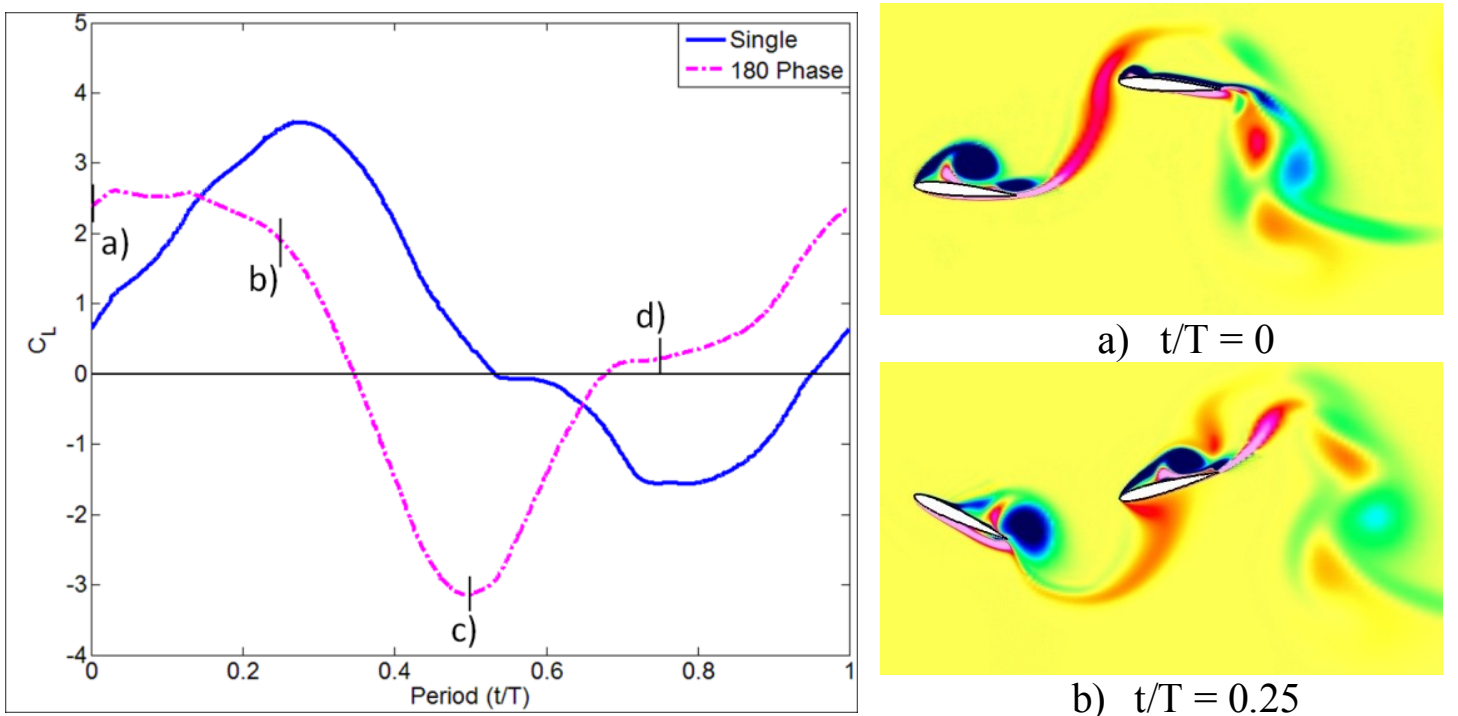

a) $t / T=0$

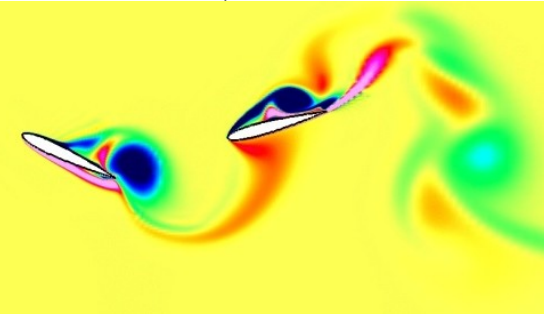

b) $\mathrm{t} / \mathrm{T}=0.25$
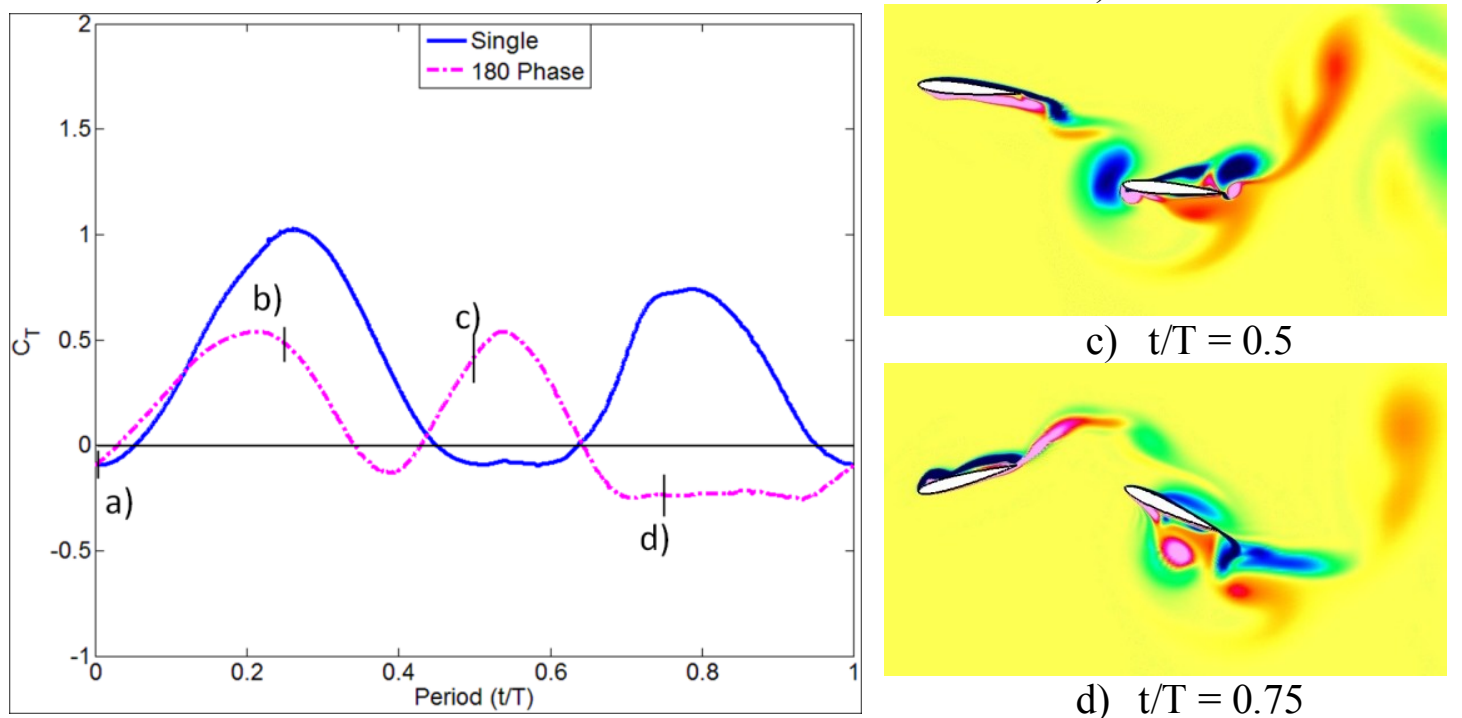

c) $\mathrm{t} / \mathrm{T}=0.5$

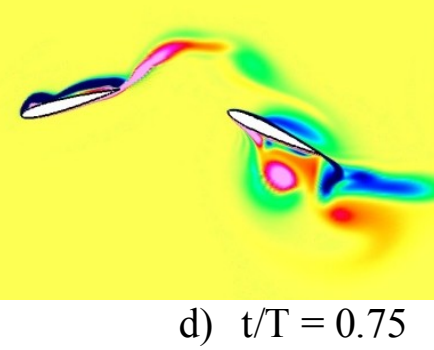

Figure 16. Lift and thrust coefficients plotted vs. time and vorticity contours at $0 \%$, $25 \%, 50 \%$ and $75 \%$ flapping cycle for the tandem wing flapping with $180^{\circ}$ phase lag. Destructive vortex interactions decrease the size of the LEVs generated by the hindwing and cause a shift in the timing of LEV formation. 
Figure 16 shows the results for the tandem wing flapping with $180^{\circ}$ phase lag. An LEV forms on the top of the hindwing during the downstroke, point a), but its formation is earlier than normal. The LEV is also smaller than normal, and while it forms on the upstream side of the airfoil, it is already convecting away as the pitch angle reaches its maximum point, at b). At these points, the force histories show decreases in the peak lift and thrust coefficients. From points c) to d), a vortex shed from the forewing bisects the hindwing. Half of it convects over the top, downstream facing side of the airfoil, while the other half detaches the LEV from the bottom, upstream facing side. This corresponds to point d) on the force graphs, where the hindwing shows positive lift and negative thrust production. This positive lift persists through the last half of the upstroke, due to the earlier than normal LEV formation on the top of the hindwing, as seen at point a). Like the $90^{\circ}$ hindwing, the large amount of positive lift produced during the upstroke is responsible for the large decrease in actuation power necessary for the hindwing at $180^{\circ}$ phase lag.

In summary, Figure 13-Figure 16 show that changing the phase angle changes the vortex interaction between the fore and hind wing. Specifically, different phase angles can be used to change the nature of LEV formation by the hindwing, which in turn affects the lift and thrust generation of the hindwing, as shown in Figure 14-Figure 16. When flapping with $0^{\circ}$ phase lag, the resulting LEV formation around the hindwing is similar to a single wing, but the LEV's are larger. This is due to the constructive vortex interactions between the fore and hind wing, where vorticity shed by the forewing interacts with like signed LEVs generated by the hindwing. Due to the larger LEV formation, the peak lift and thrust produced by the hindwing is increased. 
When the hindwing flaps with $90^{\circ}$ or $180^{\circ}$ phase lag, the vortex interactions between the fore and hind wing are destructive, where vorticity shed by the forewing interacts with opposite signed LEVs generated by the hindwing. This tends to decrease the size of the LEVs generated by the hindwing as well as change the timing of LEV formation. For the $90^{\circ}$ hindwing, LEV formation is altered to the point where it forms an LEV on the bottom of the airfoil during the downstroke and on the top of the airfoil during the upstroke. As a result, both the $90^{\circ}$ and $180^{\circ}$ see phase shifts in lift and thrust generation as well as decreases in peak lift and thrust. As a result of the phase shift in lift production, both the $90^{\circ}$ and $180^{\circ}$ hindwings produce a large amount of positive lift on the upstroke, which reduces the power coefficient of both wings. 


\section{EFFECT OF WING SPACING}

In this section, the wing spacing between the fore and hind wings is adjusted and its effect on the relationship among the phase angle, force production and efficiency is investigated using 2D numerical simulations. Three different phase angles, $0^{\circ}, 90^{\circ}$, and $180^{\circ}$ (hindwing leading) are simulated at four different wing spacings, $1.0 \mathrm{c}, 0.5 \mathrm{c}, 0.25 \mathrm{c}$, 0.1c. All cases are simulated at a Reynolds number of 5000. The shape of the airfoils is that of a flatplate with 5\% chord thickness and rounded edges. Comparisons are made to a single wing flapping with the same kinematics and at the same Reynolds number. The Strouhal number of the flapping wing kinematics is 0.3 which falls into the range of 0.2 and 0.4 used by most natural fliers ${ }^{[7]}$. Specifically, the objective of the study is to determine how changes in the wing spacing affect the resulting vortex interaction between the fore and hind wings and how that affects force generation and efficiency.

\section{A. Aerodynamic Force Results}

The lift, thrust and resultant coefficients were calculated for each wing individually using Equations 12-14. Figure 17 shows the transient lift and thrust coefficients of only the hindwing over a single flapping cycle at different wing gap spacings for the three tested phase angles, $0^{\circ}, 90^{\circ}$ and $180^{\circ}$ (hindwing leading). The same results for the single wing are also show to serve as a baseline. In the plots a cycle time, $\mathrm{t} / \mathrm{T}$, of $0 \%$ is the start of the downstroke and $50 \%$ is the start of the upstroke. 


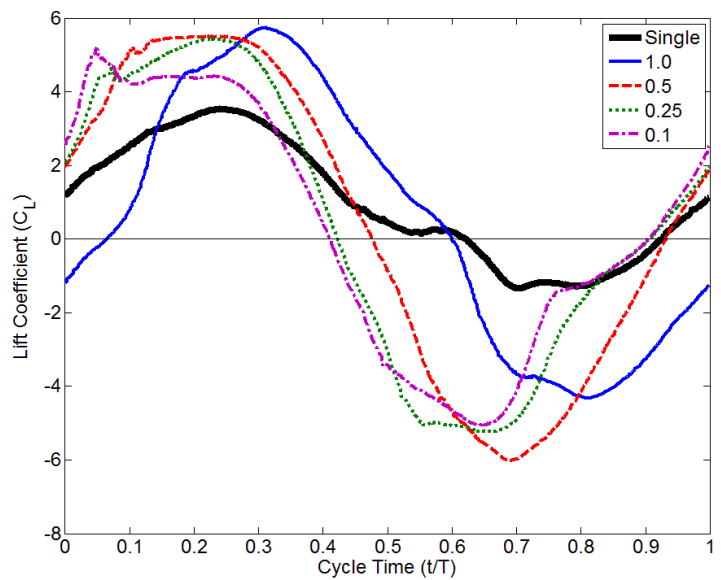

a) 0 Phase Lift

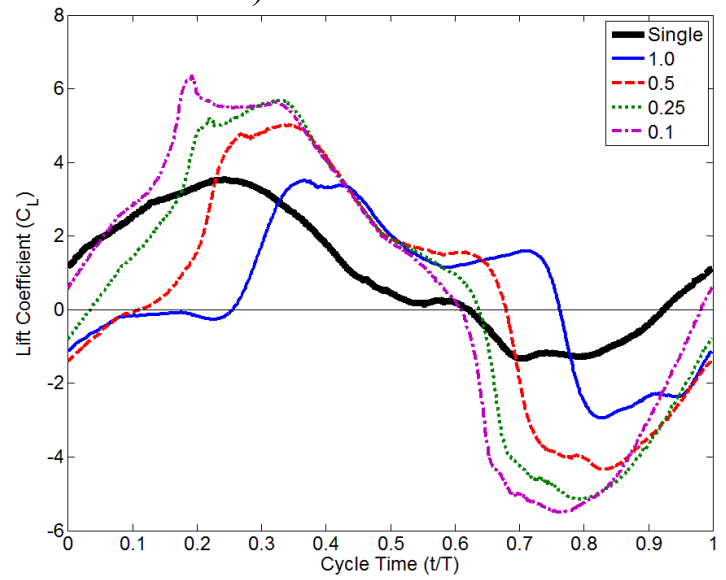

c) 90 Phase Lift

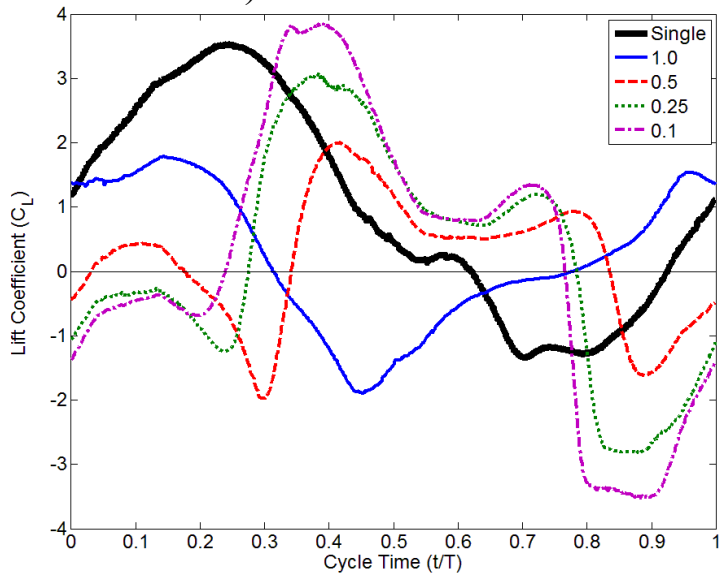

e) 180 Phase Lift

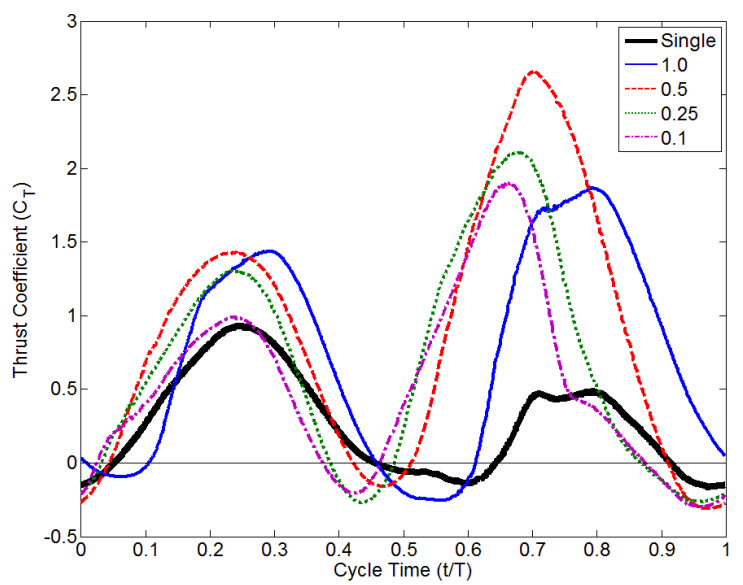

b) 0 Phase Thrust

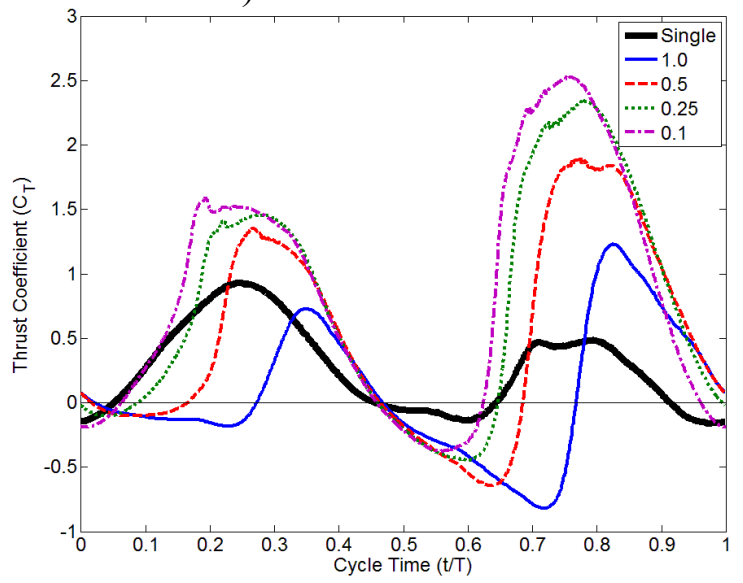

d) 90 Phase Thrust

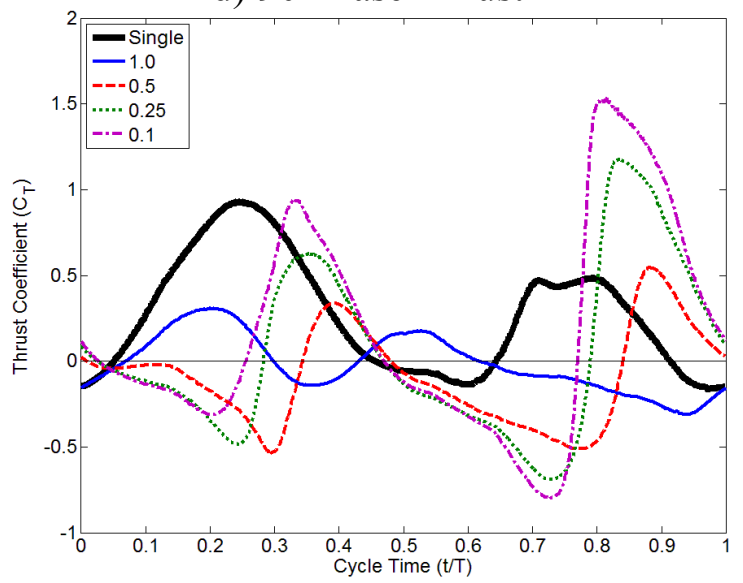

f) 180 Phase Thrust

Figure 17. Transient lift and thrust coefficients for the hindwing over a single flapping cycle compared to a single wing. Each graph shows the lift or thrust at a single phase angle for the four tested spacings.

The results in Figure 17 illustrate the significant effect that changing the phase angle or wing spacing can have on the lift and thrust generation of the hindwing. The 
effect of the phase angle is considered first. At $0^{\circ}$ phase angle, the lift and thrust amplitudes of the hindwing are much higher than the single wing case at each spacing. When the phase angle is $90^{\circ}$, the hindwing has higher force amplitudes than the single wing at the small spacings but not at the large spacing of $1.0 \mathrm{c}$. Finally, at $180^{\circ}$ phase lag, the hindwing has higher force amplitudes than the single wing through the upstroke but lower force amplitudes during the downstroke for small spacings. At the largest spacing, however, the hindwing has significantly lower force amplitudes than the single wing.

Next, the impact of the wing spacing is considered. In general, increasing the wing spacing causes a phase lag in both lift and thrust generation. This trend is shown clearly in the case where the fore and hind wings flap with a $90^{\circ}$ phase lag. Both the lift and thrust show a clear phase lag in the timing of the lift and thrust generation as the spacing is increased from $0.1 \mathrm{c}$ to $1.0 \mathrm{c}$. There is also a trend of increasing lift and thrust amplitudes as the spacing is decreased. The $180^{\circ}$ phase case shows nearly the same trend as the $90^{\circ}$ phase case. There is a phase lag in both the lift and thrust as the spacing is increased from $0.1 \mathrm{c}$ to $0.5 \mathrm{c}$, just as at $90^{\circ}$, but increasing the spacing to $1.0 \mathrm{c}$ does not show the same trend in the phase lag. The $180^{\circ}$ case also exhibits increases in the peak lift and thrust as the spacing is decreased, similar to the $90^{\circ}$ case. The trends observed in the $90^{\circ}$ and $180^{\circ}$ cases are not as evident for the $0^{\circ}$ case. While the $0^{\circ}$ case exhibits phase lag in the timing of the force generation as the spacing is decreased, it is inconsistent. Also, the peak lift and thrust magnitudes do not show the same increasing trend that is observed with the $90^{\circ}$ and $180^{\circ}$ cases as the spacing is decreased. 


\section{B. Flow Visualization Results}

The behavior observed in the force data can be explained by analyzing the vorticity contours for each case. Specifically, examining how changes in the phase angle and wing spacing changes the timing of vortex interactions during the flapping cycle of the hindwing. Figure 18, Figure 19, and Figure 20 show the vorticity contours at different spacings for the $90^{\circ}, 180^{\circ}$ and $0^{\circ}$ cases, respectively. For the sake of brevity, the vorticity contours are only shown for one half of the cycle (upstroke for the $90^{\circ}$ and $180^{\circ}$ cases and downstroke for the $0^{\circ}$ case) as the upstroke and downstroke exhibit nearly symmetric results.

Figure 18 illustrates how changes in the wing spacing affect the timing of the vortex/wing and vortex/vortex interactions and how the interactions influence leading edge vortex (LEV) formation during the upstroke of the hindwing when the wings flap with $90^{\circ}$ phase lag. During the upstroke, the hindwing passes through a vortex shed from the trailing edge of the forewing. At the closer spacings this interaction occurs during the first half of the upstroke ( $68 \%$ cycle time). As the spacing is increased, this vortex interaction is delayed, due to the increased time necessary for the vortex to convect to the hindwing, and occurs later in the upstroke. This delay in the vortex interaction is clearly observed as a phase lag in the transient lift and thrust data shown in Figure 17(c) and (d).

The timing of the interaction between the hindwing and vortex shed from the forewing has a noticeable effect on the LEV formation around the hindwing. The bottom row of Figure 18 shows the vorticity contours when the hindwing is at $89 \%$ cycle, just before stroke reversal, after the vortex interaction has occurred. The rotation of the shed vortex is the same as the rotation of the LEV that forms on the bottom of the hindwing 
(Counterclockwise, $\mathrm{CCW}$ ), which reinforces the LEV generation. At the closer spacings, the vortex interaction is stronger because the vortex shed from the forewing has less time to dissipate before interacting with the hindwing. This results in larger LEV generation by the hindwing, which corresponds with the increased peak in the transient lift and thrust data as the spacing is decreased. The increased peak in the transient force data can be observed in Figure 17(c) and (d). On the downstroke, the same interaction and phase lag is observed, except that the vortex shed from the forewing and the LEV generated by the hindwing are clockwise $(\mathrm{CW})$. 


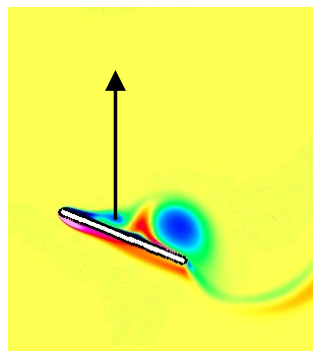

a) Single, $\mathrm{t} / \mathrm{T}=68 \%$

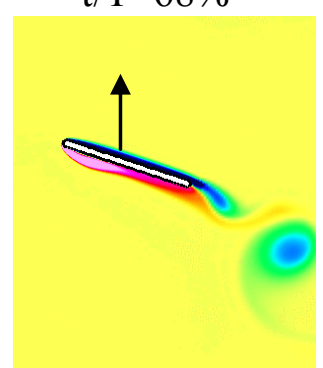

e) Single, $\mathrm{t} / \mathrm{T}=89 \%$

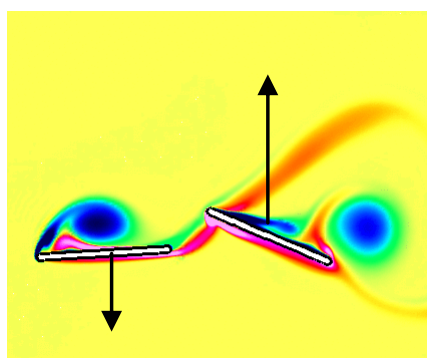

b) $0.25 \mathrm{c}, \mathrm{t} / \mathrm{T}=68 \%$

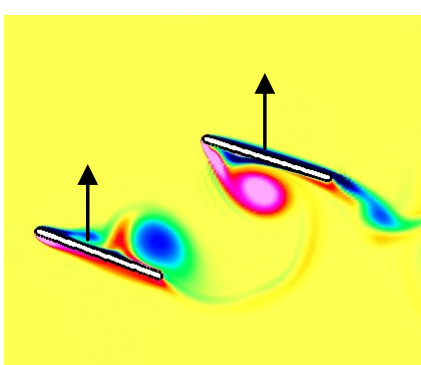

f) $0.25 \mathrm{c}, \mathrm{t} / \mathrm{T}=89 \%$

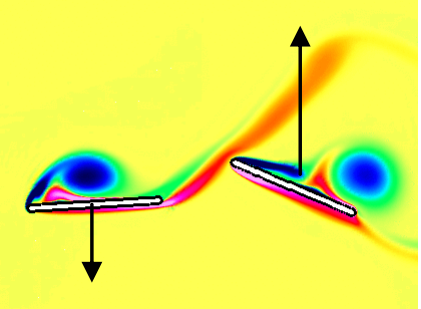

c) $0.5 \mathrm{c}, \mathrm{t} / \mathrm{T}=68 \%$

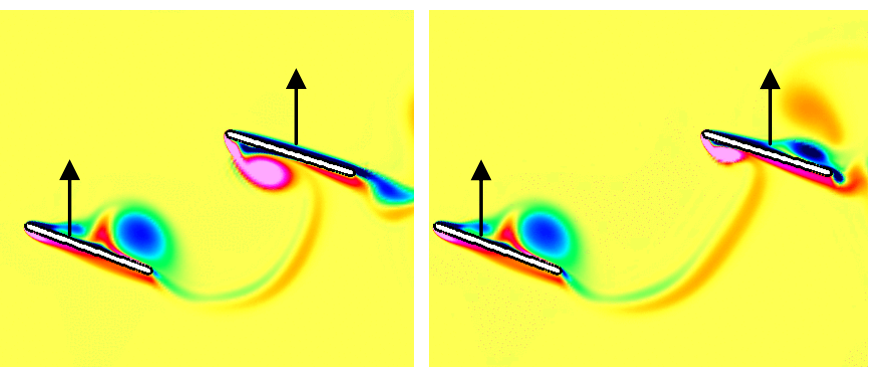

g) $0.5 \mathrm{c}, \mathrm{t} / \mathrm{T}=89 \%$ d) $1.0 \mathrm{c}, \mathrm{t} / \mathrm{T}=68 \%$

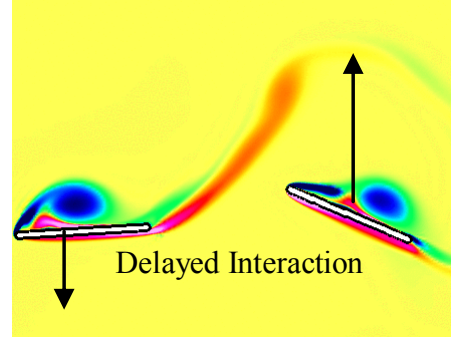

h) $1.0 \mathrm{c}, \mathrm{t} / \mathrm{T}=89 \%$

Figure 18. The vorticity contours of the single wing and the $90^{\circ}$ phase angle tandem configuration at different spacings. The large spacing delays and weakens the vortex/wing interaction. The first row shows the single and hindwing during the upstroke at $68 \%$ cycle time while the second row shows the single and hindwing at $89 \%$ cycle time. Arrows indicate the stroke direction. 
Similar to Figure 18, Figure 19 shows the vorticity contours at different spacings for the $180^{\circ}$ phase case during the upstroke. In this case, the hindwing passes through the vortex shed by the forewing, as in the $90^{\circ}$ case, but the vortex interaction occurs significantly later in the upstroke due to a larger phase angle. At the closest spacings, the hindwing starts to interact with the shed vortex in the second half of the upstroke, at $81 \%$ of cycle compared to $68 \%$ of cycle for $90^{\circ}$ case. As the spacing is increased this interaction is delayed, which corresponds with the phase lag in the force data shown in Figure $17(\mathrm{e})$ and (f). This behavior is similar to that observed in the $90^{\circ}$ case. At $180^{\circ}$ phase lag, however; when the wing spacing is increased to $1.0 \mathrm{c}$, the vortex interaction is delayed until after stroke reversal, which allows the start of LEV formation on top of the hindwing. The delay is reflected in the force data shown in Figure 17(e) and (f), in which the 1.0c spacing shows dramatically different pattern from other spacings.

The timing of the vortex interaction has large implications on the LEV generation of the hindwing. These implications can be observed by comparing the LEVs on the hindwings in Figure 19(e-h). At the closest spacings, the hindwing passes through the shed vortex before stroke reversal, which serves to reinforce the LEV formation at the bottom of the hindwing (both vortices have the same rotation, $\mathrm{CCW}$ ). As the spacing is increased, the vortex interaction becomes weaker as the vortex shed from the forewing has more time to dissipate before interacting with the hindwing, resulting in smaller LEV generation. This corresponds with the lower peak lift and thrust observed in the force data as the spacing is increased as observed in Figure 17(e) and (f). At the spacing of 1.0c, the interaction is delayed until stroke reversal, where the hindwing starts to form a CW LEV on top. The interaction with the CCW shed vortex dampens out the CW LEV 
and quickens its shedding, which results in the extremely low lift and thrust production observed for this case in Figure 17(e) and (f). As with the $90^{\circ}$ case, the result on the downstroke are symmetrical to the upstroke except that the rotation of the vortices are reversed. 


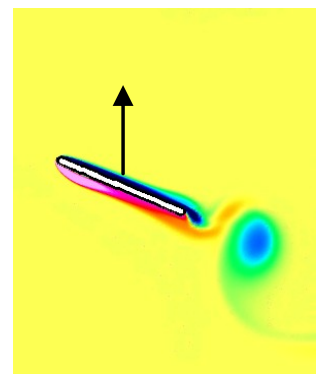

a) Single, $\mathrm{t} / \mathrm{T}=81 \%$

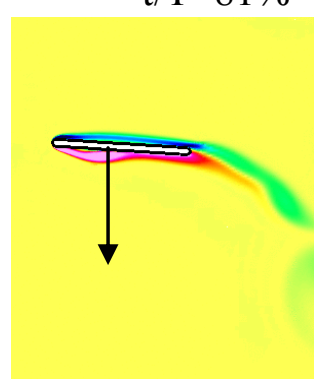

e) Single,

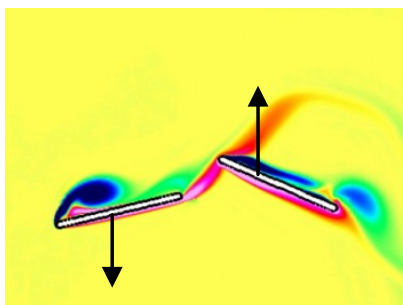

b) $0.25 \mathrm{c}, \mathrm{t} / \mathrm{T}=81 \%$

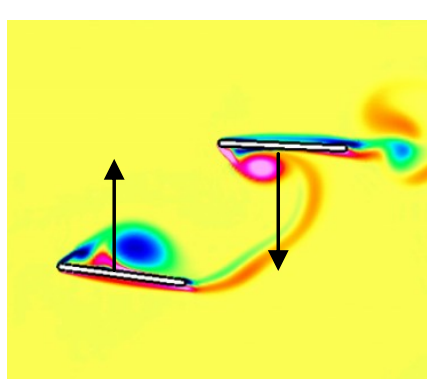

f) $0.25 \mathrm{c}, \mathrm{t} / \mathrm{T}=2 \%$

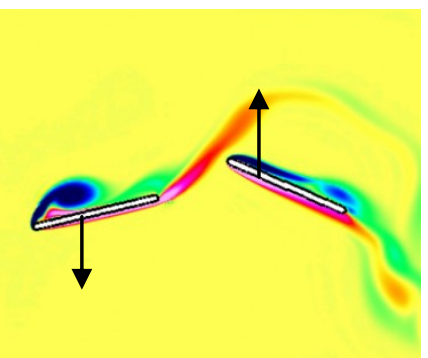

c) $0.50 \mathrm{c}, \mathrm{t} / \mathrm{T}=81 \%$

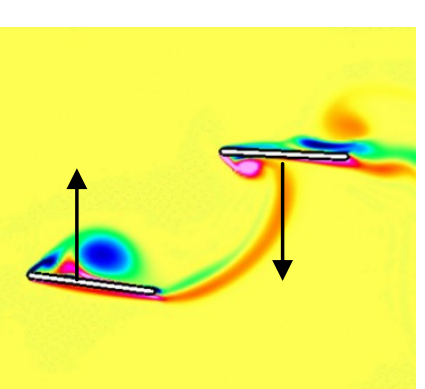

g) $0.5 \mathrm{c}, \mathrm{t} / \mathrm{T}=2 \%$

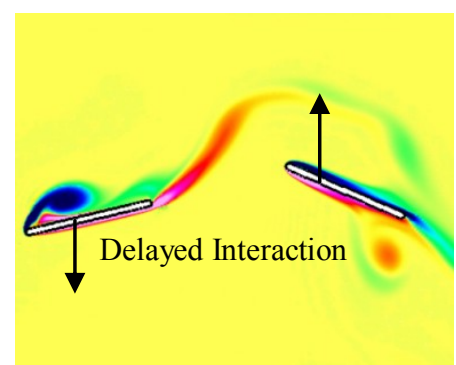

d) $1.0 \mathrm{c}, \mathrm{t} / \mathrm{T}=81 \%$

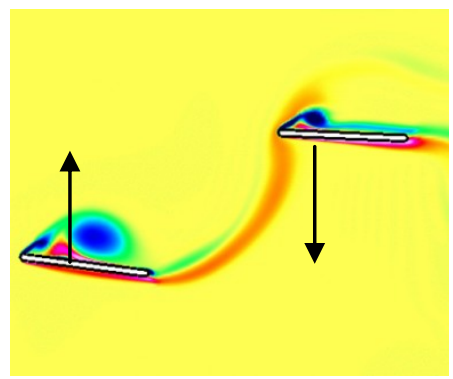

h) $1.0 \mathrm{c}, \mathrm{t} / \mathrm{T}=2 \%$

Figure 19. The vorticity contours of the single wing and the $180^{\circ}$ phase angle tandem configuration at different spacings. The vortex/wing interaction is delayed compared to the $90^{\circ}$ phase angle tandem configuration case. The first row shows the single and hindwing during the upstroke at $81 \%$ cycle time while the second row shows the single and hindwing at $2 \%$ cycle time. Arrows indicate the stroke direction. 
Figure 20 shows the vorticity contours at the tested spacings for the $0^{\circ}$ phase case during the downstroke. The results are shown for the downstroke rather than the upstroke because the vortex interaction is easier to observe. Similar to the $90^{\circ}$ and $180^{\circ}$ cases, the hindwing passes through the vortex shed from the trailing edge of the forewing and the interaction is delayed as the spacing is increased. Because the fore and hind wings flap with $0^{\circ}$ phase lag, at the smaller spacings, the two wings remain in close proximity to each other throughout the entire cycle. At the $1.0 \mathrm{c}$ and $0.5 \mathrm{c}$, the interaction between the CW shed vortex and the hindwing reinforces the formation of the CW LEV on the hindwing, which results in increased peak lift and thrust. At the closest spacings, however; a jet forms between the two plates, which quickens the LEV shedding and results in a smaller and elongated LEV at the closest spacings. This behavior is most obvious when the spacing is decreased to 0.1c (which is shown in Figure 20, rather than $0.25 \mathrm{c}$ ). Figure 21 shows the vertical velocity profile of this jet (between the trailing edge of the forewing and leading edge of the hindwing) for the $0.1 \mathrm{c}$ and $1.0 \mathrm{c}$ cases at $12 \%$ cycle time (the first half of the downstroke). It is clear that at the closer spacing the vertical velocity component of the jet is much stronger. The weakened LEV production at the closest spacings explains why the peak lift and thrust does not continue to increase as the spacing is decreased like the $90^{\circ}$ and $180^{\circ}$ cases. 


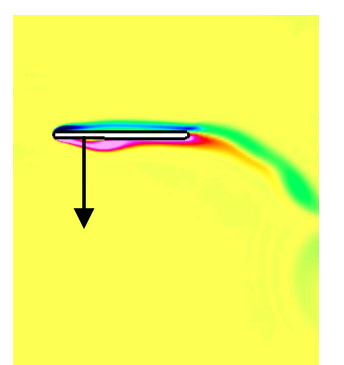

a) Single, $\mathrm{t} / \mathrm{T}=4 \%$

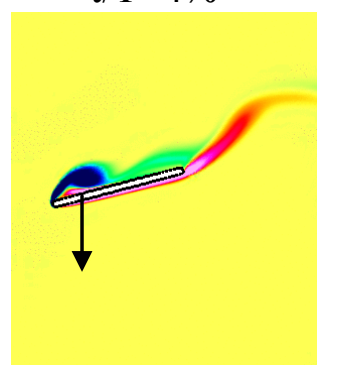

e) Single, $\mathrm{t} / \mathrm{T}=25 \%$

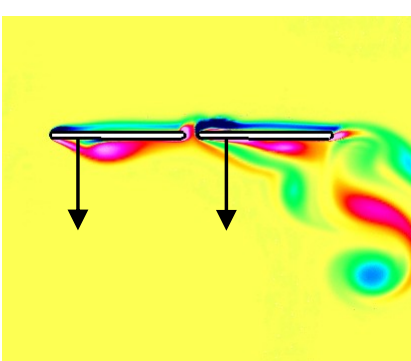

b) $0.1 \mathrm{c}, \mathrm{t} / \mathrm{T}=4 \%$

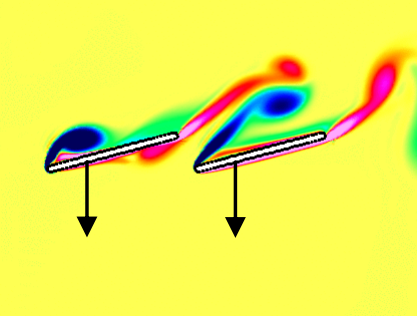

f) $0.1 \mathrm{c}, \mathrm{t} / \mathrm{T}=25 \%$ c) $0.5 \mathrm{c}, \mathrm{t} / \mathrm{T}=4 \%$
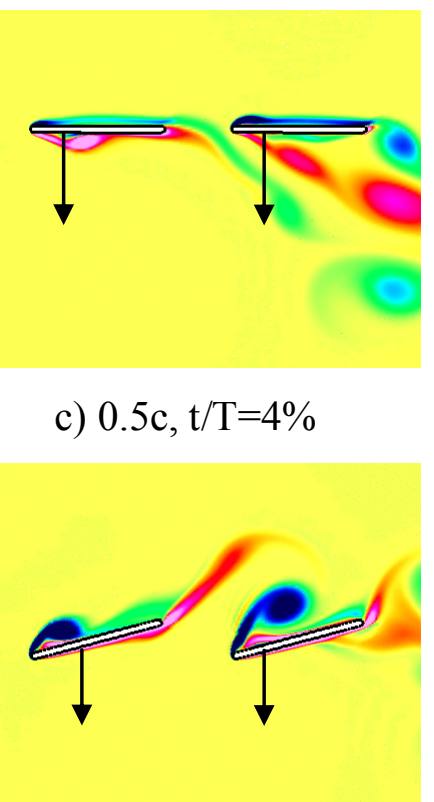

g) $0.5 \mathrm{c}, \mathrm{t} / \mathrm{T}=25 \%$
Delayed Interaction

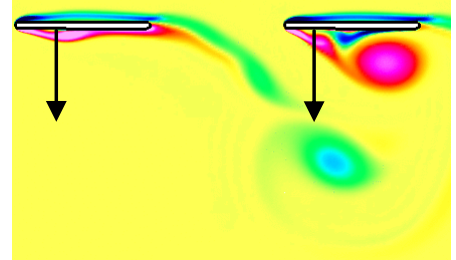

d) $1.0 \mathrm{c}, \mathrm{t} / \mathrm{T}=4 \%$

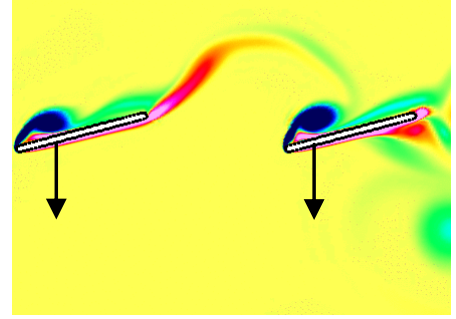

h) $1.0 \mathrm{c}, \mathrm{t} / \mathrm{T}=25 \%$

Figure 20. The vorticity contours of the single wing and the $0^{\circ}$ tandem configuration at different spacings. The first row shows the single and hindwing during the downstroke at $4 \%$ cycle time while the second row shows the single and hindwing at $25 \%$ cycle time. Arrows indicate the stroke direction. 


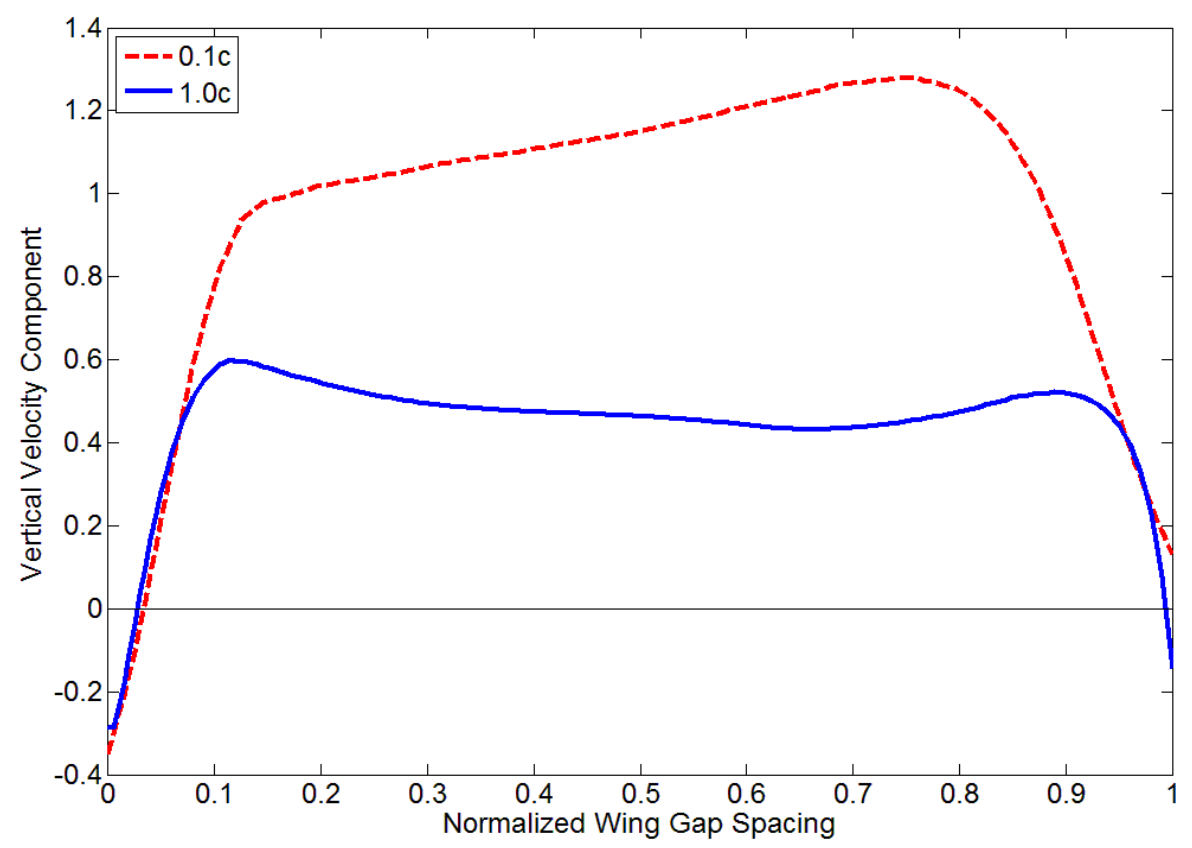

Figure 21. The profile of the vertical velocity component taken between the trailing edge of the forewing and the leading edge of the hindwing. The results shown are for a gap spacing of $0.1 \mathrm{c}$ and $1.0 \mathrm{c}$ with the $\mathrm{x}$-axis normalized by the wing spacing.

\section{Effect of Vortex Interaction on $\mathbf{C}_{\mathbf{P}}$}

The effect of the vortex interaction on the pressure distribution around the airfoil is shown in Figure 22.

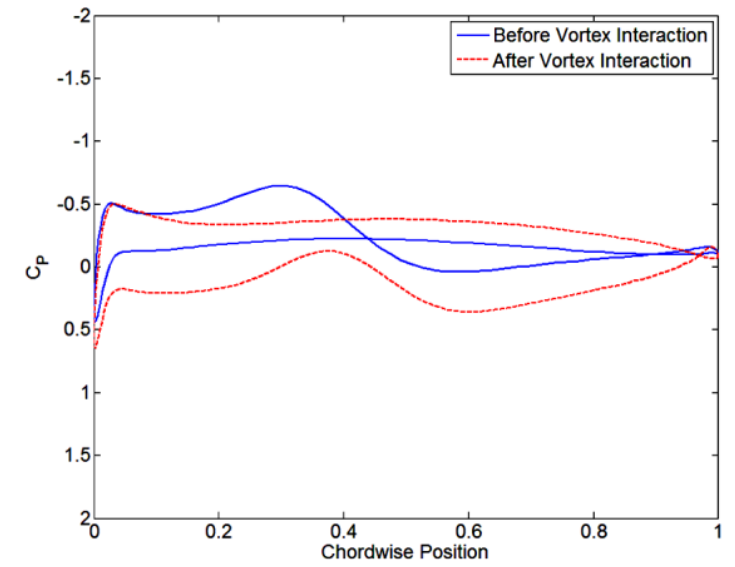

(a)

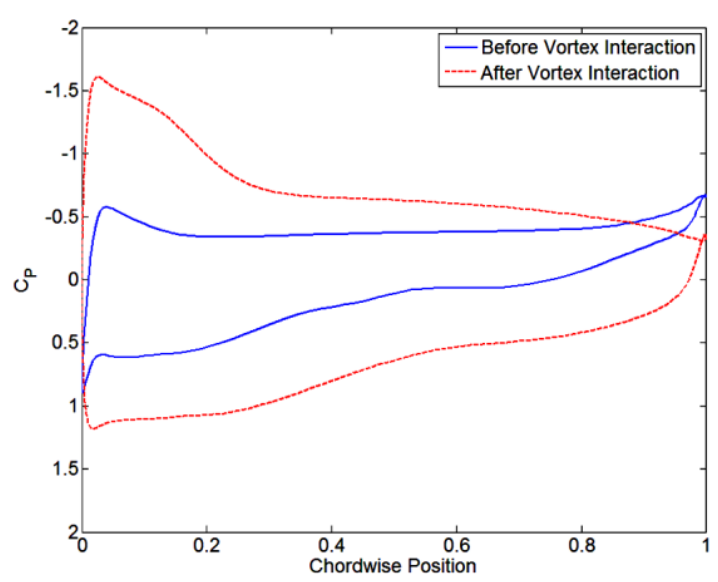

(b)

Figure 22. There is a large increase in suction on the top of the leading edge of the hindwing that is associated with the passing of the vortex shed by the forewing. There is no observable increase for the single wing. 
The y-axis is reversed so that the top of the curve corresponds to the top of the airfoil (negative pressure) and vice versa. Figure 22 compares the pressure distribution around the airfoil of both the single wing (a) and the hind wing at a phase angle of $0^{\circ}$ and a wing spacing of $0.5 \mathrm{c}$ (b) immediately before and immediately after the vortex interaction during the downstroke, which is the vortex interaction shown in Figure 20. The hindwing shows a large increase in suction at the top of the leading edge that corresponds with the passing of the vortex shed by the forewing. There is no observable increase in suction for the single wing at the same point in the cycle.

\section{Effect of Phase angle vs. Spacing}

Figure 23 compares the transient lift and thrust history of the $0^{\circ}$ phase angle case at $1.0 \mathrm{c}$ to the $90^{\circ}$ case at $1.0 \mathrm{c}$ and $0.25 \mathrm{c}$. Despite the difference in phase angle, the $0^{\circ}$ case at $1.0 \mathrm{c}$ and the $90^{\circ}$ case at $0.25 \mathrm{c}$ exhibit remarkably similar force histories. These two cases show nearly the same trend in lift and thrust generation (hence vortex and wing interactions) while the only difference between them is in peak lift and thrust production. This reveals that, in terms of force generation, decreasing the wing spacing has the opposite effect as increasing the phase angle. In this case, decreasing the baseline spacing from $1.0 \mathrm{c}$ to $0.25 \mathrm{c}$ while also increasing the baseline phase angle from $0^{\circ}$ to $90^{\circ}$ generates similar results as the baseline. However, when the phase angle is increased from $0^{\circ}$ to $90^{\circ}$ while keeping the spacing constant at $1.0 \mathrm{c}$, there is a significant change in the transient lift and thrust coefficient compared to the other two cases. 


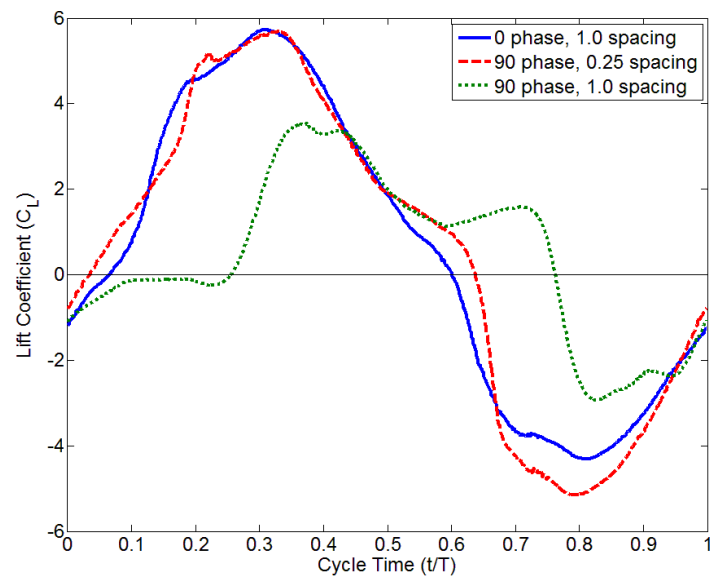

a) Lift

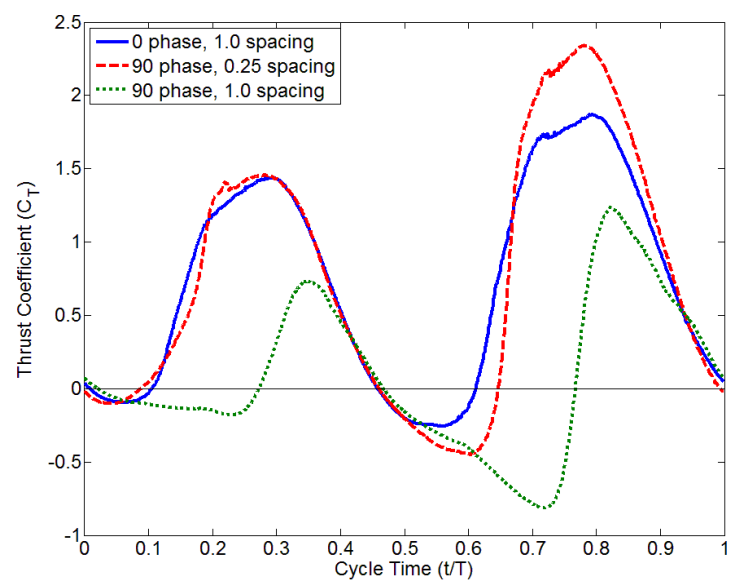

b) Thrust

Figure 23. Comparison of the lift and thrust over a single flapping cycle for the $0^{\circ}$ hindwing at $1.0 \mathrm{c}$ to the $90^{\circ}$ hindwing at $1.0 \mathrm{c}$ and $0.25 \mathrm{c}$.

Figure 24 is similar to Figure 23, except it compares the force history of the $90^{\circ}$ phase angle case at $1.0 \mathrm{c}$ to the $180^{\circ}$ case at $1.0 \mathrm{c}$ and $0.25 \mathrm{c}$. Again, the resulting force histories are very similar when the phase angle is increased by $90^{\circ}$ while simultaneously decreasing the spacing from $1.0 \mathrm{c}$ to $0.25 \mathrm{c}$. The two cases $\left(90^{\circ}\right.$ at $1.0 \mathrm{c}$ and $180^{\circ}$ at $\left.0.25 \mathrm{c}\right)$ show similar timing in force generation, but different magnitudes of peak lift and thrust. Like Figure 23, increasing the phase angle from $0^{\circ}(1.0 \mathrm{c})$ to $90^{\circ}(1.0 \mathrm{c})$ causes a shift in force histories. However, decreasing the spacing from $1.0 \mathrm{c}\left(90^{\circ}\right)$ to $0.25 \mathrm{c}\left(90^{\circ}\right)$ will offset the shift. This shows that increasing the phase angle has the opposite effect as decreasing the spacing. 


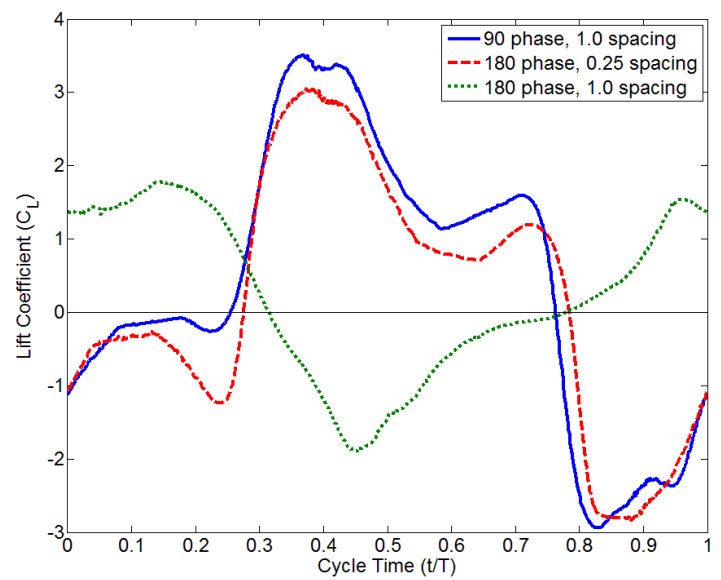

a) Lift

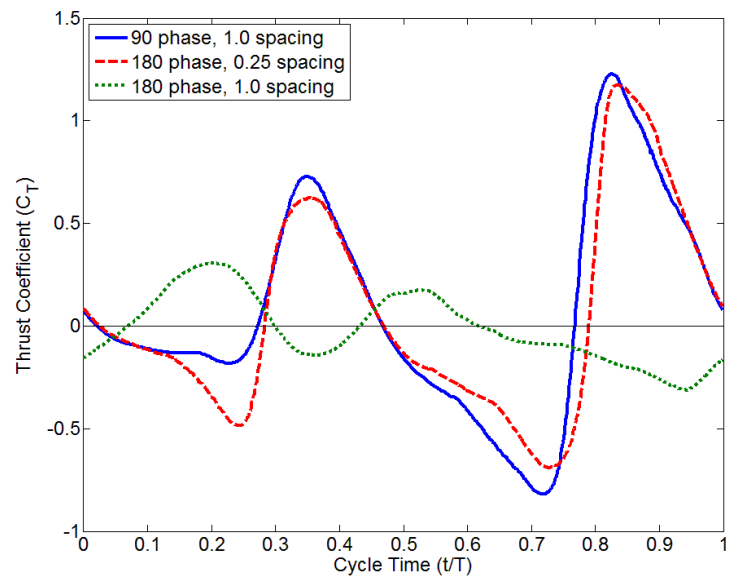

b) Thrust

Figure 24. Comparison of the lift and thrust over a single flapping cycle for the $90^{\circ}$ hindwing at $1.0 \mathrm{c}$ to the $180^{\circ}$ hindwing at $1.0 \mathrm{c}$ and $0.25 \mathrm{c}$.

Examining the vorticity contours reveals why the force histories are nearly the same between the different cases. Figure 25 compares the vorticity contours between the $0^{\circ}$ and $90^{\circ}$ cases at the different spacings during the upstroke. For these specific cases, the hindwing passes through the vortex shed from the forewing at nearly the same time during the upstroke, which results in similar LEV generation on the hindwing. Specifically, the timing of LEV generation and shedding is approximately the same among the two cases despite the difference in spacing and phase angle. This corresponds to the similar force histories observed in Figure 24. There are slight differences in the size of the LEV, which results in the difference in peak lift and thrust observed between the two cases. 


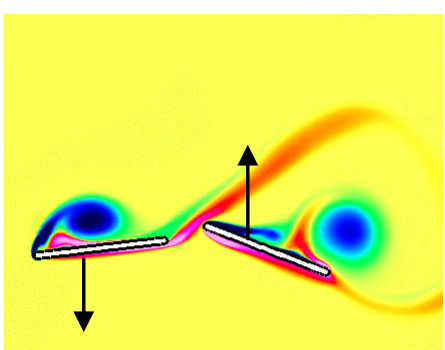

a) $90^{\circ}, 0.25 \mathrm{c}, \mathrm{t} / \mathrm{T}=65 \%$

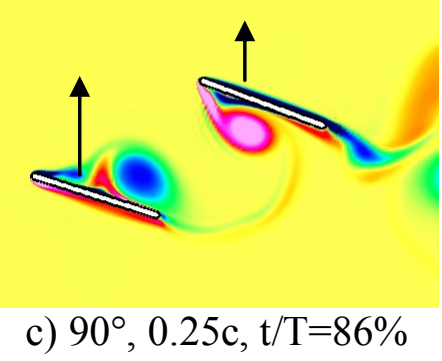

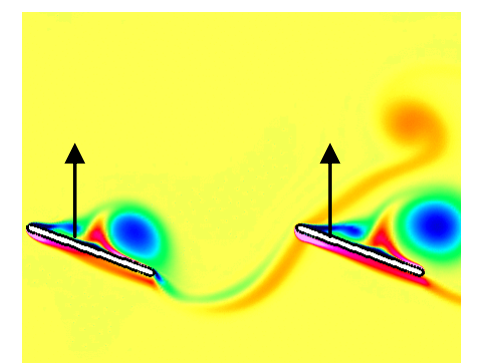

b) $0^{\circ}, 1.0 \mathrm{c}, \mathrm{t} / \mathrm{T}=65 \%$

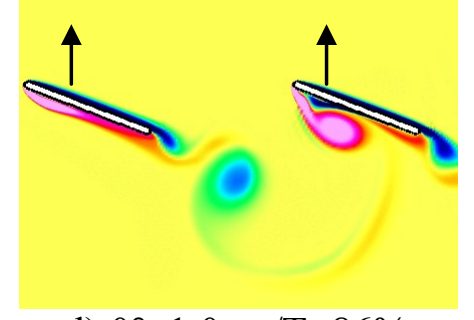

d) $0^{\circ}, 1.0 \mathrm{c}, \mathrm{t} / \mathrm{T}=86 \%$

Figure 25. Comparison of the vorticity contours for the $0^{\circ}$ hindwing at $1.0 \mathrm{c}$ to the $90^{\circ}$ hindwing at $0.25 \mathrm{c}$ and $0.1 \mathrm{c}$. Despite the parameter difference, the vortex structures on the hind wing are very similar. The contours were taken on the upstroke at $65 \%$ and $86 \%$ of the cycle time. Arrows indicate the stroke direction.

Figure 26 shows similar results to Figure 25 for the $180^{\circ}$ and $90^{\circ}$ cases. For these two cases, though the phase angle and spacing are not the same, the timing of the vortex interaction between the fore and hind wings during the upstroke of the hindwing is nearly identical. This results in comparable LEV formation on the hindwing and the resulting similarities in the transient lift and thrust data between the $90^{\circ}$ and $180^{\circ}$ cases shown in Figure 24. As with the $0^{\circ}$ and $90^{\circ}$ cases, there are slight differences in the size of the LEV generated, which result in the differences observed in the peak lift and thrust. 


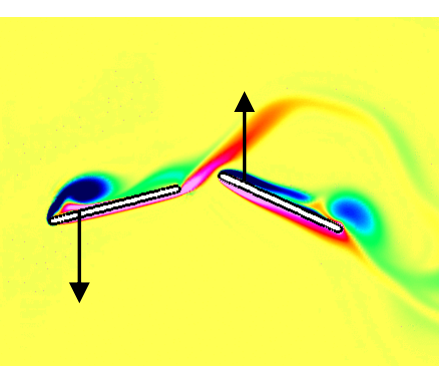

a) $180^{\circ}, 0.25 \mathrm{c}, \mathrm{t} / \mathrm{T}=77 \%$

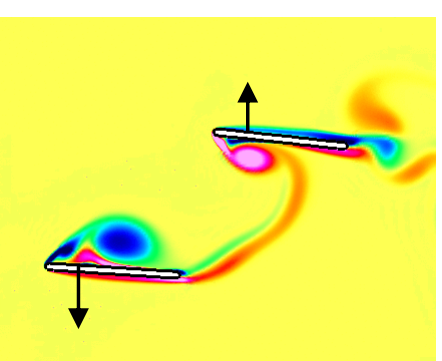

c) $180^{\circ}, 0.25 \mathrm{c}, \mathrm{t} / \mathrm{T}=98 \%$

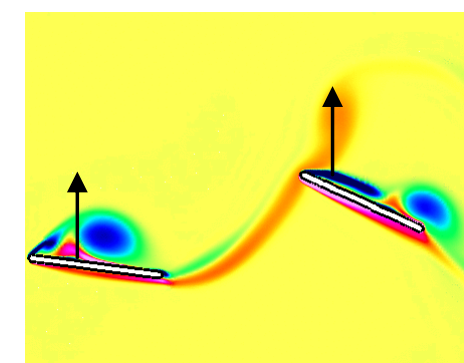

b) $90^{\circ}, 1.0 \mathrm{c}, \mathrm{t} / \mathrm{T}=77 \%$
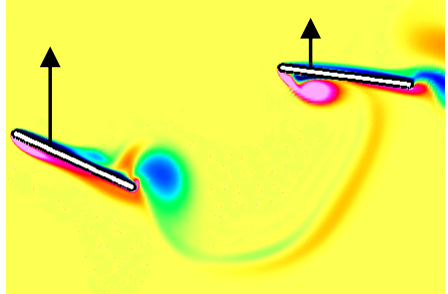

d) $90^{\circ}, 1.0 \mathrm{c}, \mathrm{t} / \mathrm{T}=98 \%$

Figure 26. Comparison of the vorticity contours for the $0^{\circ}$ hindwing at $1.0 \mathrm{c}$ to the $90^{\circ}$ hindwing at $0.25 \mathrm{c}$ and 0.1c. Again, vortex structures on the hindwing are very similar, despite the parameter difference. The contours were taken on the upstroke at $77 \%$ and $98 \%$ of the cycle time. Arrows indicate the stroke direction.

\section{E. Cycle Averaged Force Results}

The cycle averaged lift, thrust and resultant coefficients were calculated over a single flapping cycle for the hindwing of each phase angle and plotted versus the spacing. These, along with the time averaged power coefficient and the lift and propulsive efficiencies, are shown in Figure 27. The cycle averged power coefficient, which is a nondimensional measurement of the power required to actuate the wing during a single flapping cycle, was calculated using Equation 18. The lift and propulsive efficiencies were calculated using Equations 19-20. 


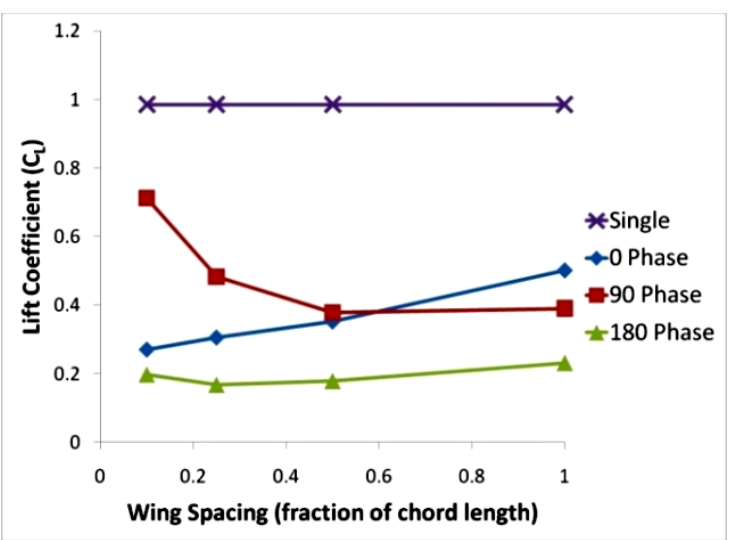

a) Lift

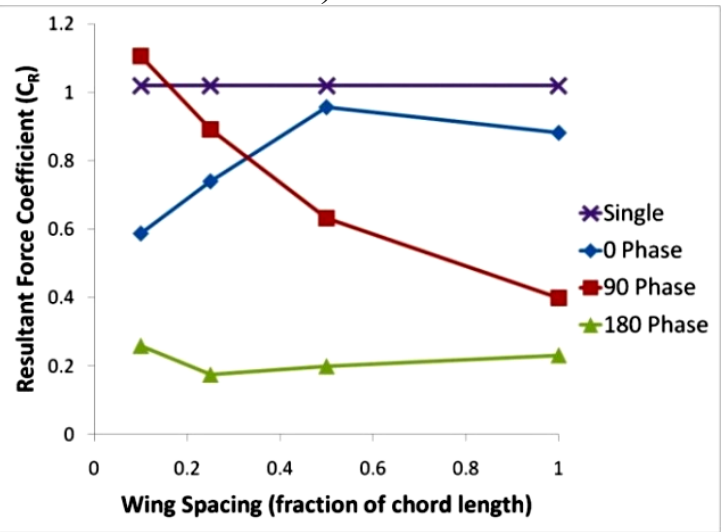

c) Resultant

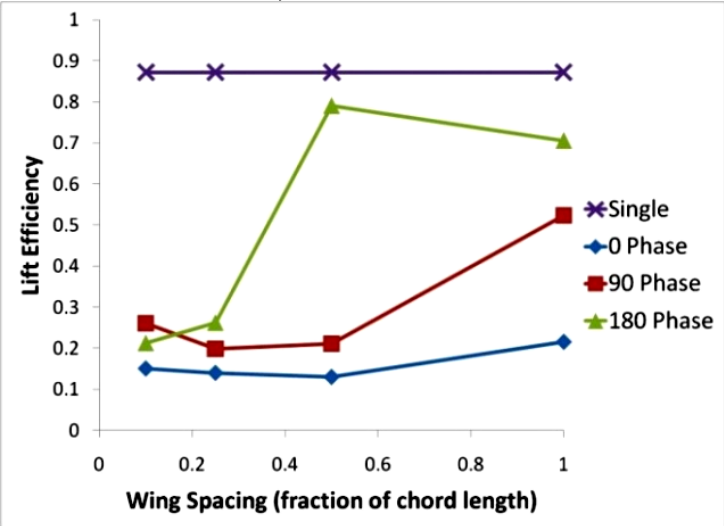

e) Lift Efficiency

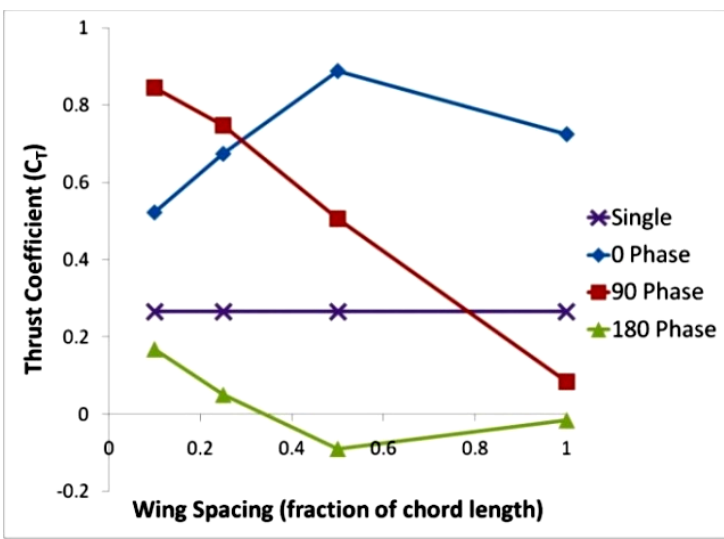

b) Thrust

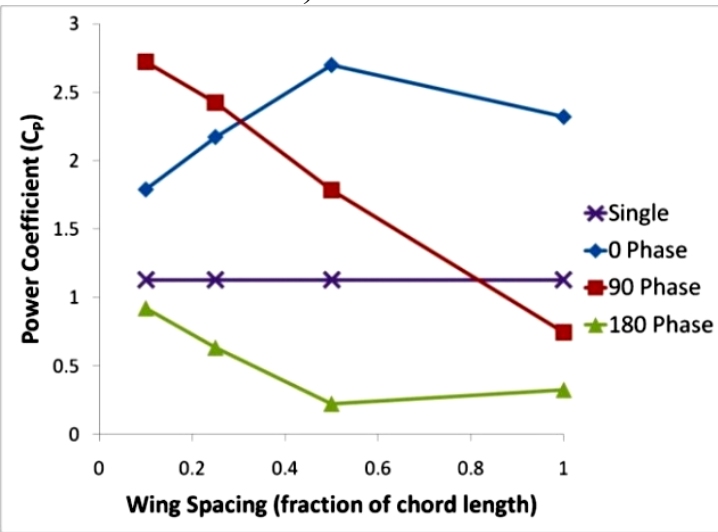

d) Power

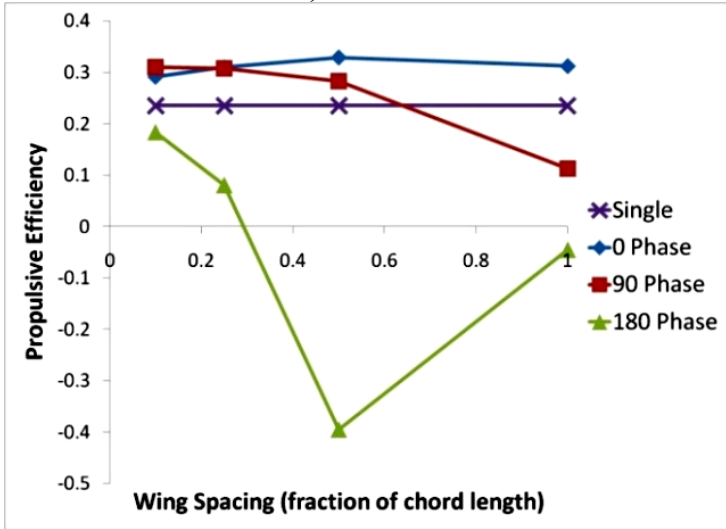

f) Propulsive Efficiency

Figure 27. Cycle averaged lift, thrust, resultant and power coefficients as well as the lift and propulsive efficiency for the hindwing. The results are graphed vs. the spacing for the three phase angles $\left(0^{\circ}, 90^{\circ}, 180^{\circ}\right)$ and compared to the single wing.

Figure 27 demonstrates how the phase angle and spacing affect the cycle averaged force production and power consumption, as well as the efficiencies. The results for a single wing are also included to provide a baseline comparison. As shown in Figure 
27(a) the hindwing has a lower lift coefficient than a single wing at all tested cases. For the $0^{\circ}$ and $180^{\circ}$ phase angles, the lift coefficient decreases as the spacing is decreased, but for the $90^{\circ}$ phase angle case, the lift coefficient increases as the spacing decreases.

The hindwing of the $0^{\circ}$ and $90^{\circ}$ cases consistently have a significantly higher thrust coefficient (Figure 27(b)) than a single wing, while the $180^{\circ}$ hindwing has a much lower thrust coefficient than a single wing, and actually produces net drag at the farthest two spacings $(0.5 \mathrm{c}$ and $1.0 \mathrm{c})$. The $90^{\circ}$ cases shows the most significant change in the thrust coefficient as the spacing is changed, rising nearly linearly from 0.07 at $1.0 \mathrm{c}$ to 0.82 at $0.1 \mathrm{c}$. The $0^{\circ}$ case shows a peak in the thrust coefficient at $0.5 \mathrm{c}$ and then a noticeable decrease as the spacing is increased farther, while the $180^{\circ}$ case shows a minimum thrust coefficient at $0.5 \mathrm{c}$ and then a significant rise in the thrust coefficient as the spacing is decreased. The resultant force coefficient (Figure 27(c)) and power coefficient (Figure 27(d)) of each phase angle shows a similar trend to that observed with the thrust coefficient, except that the resultant coefficient of all cases is lower than the resultant coefficient of a single wing (due to the low lift production of the hindwing in all cases), except for the resultant coefficient of the $90^{\circ}$ hindwing at $0.1 \mathrm{c}$.

In terms of efficiency, the $180^{\circ}$ case has the highest lift efficiency at each spacing (but still less than a single wing) while the $0^{\circ}$ case has the lowest, except at a spacing of $0.1 \mathrm{c}$ where the $90^{\circ}$ case has the highest lift efficiency. The $0^{\circ}$ and $90^{\circ}$ cases exhibit the highest propulsive efficiencies (more than a single wing) while the $180^{\circ}$ case has the lowest (less than a single wing). The propulsive efficiency of the $0^{\circ}$ case is nearly constant at all spacings while the $90^{\circ}$ case shows a significant increase in efficiency with decreased spacing. The $180^{\circ}$ hindwing has a negative propulsive efficiency at the largest 
two spacings (net drag production), with a minimum at $0.5 \mathrm{c}$, but its efficiency increases to just slightly less than the single wing at $0.1 \mathrm{c}$.

It is interesting to note how changes in the spacing affect the relationship between phase angle and force production. At the largest spacing, $1.0 \mathrm{c}$, the hindwing at $0^{\circ}$ phase lag produces the largest lift and thrust coefficients, while the hindwing at $180^{\circ}$ phase lag produces the smallest with the $90^{\circ}$ hindwing falling in between the two. As the spacing is decreased, the lift and thrust coefficients rise for the $90^{\circ}$ hindwing and fall for the $0^{\circ}$ hindwing. At a spacing of $0.1 \mathrm{c}$, the $180^{\circ}$ hindwing still produces the smallest lift and thrust coefficients, but the $90^{\circ}$ hindwing has the largest lift and thrust coefficients, while the $0^{\circ}$ hindwing falls between the two. This certainly shows that the relationship between the phase angle and the force production is not constant with wing spacing.

The time averaged values of the force and power coefficients were also averaged between the fore and hind wings in order to determine the results for the tandem configuration as a complete system. These results were also plotted for each phase angle versus the spacing in Figure 28 to show the effect of phase angle and spacing on the performance of the entire system. For this comparison, the lift thrust and resultant coefficients were calculated using Equations 22-24. Since the combined force production of the tandem configuration is normalized by the planform area of both wings, the lift, thrust and resultant coefficients can be compared to the same results for a single wing. 


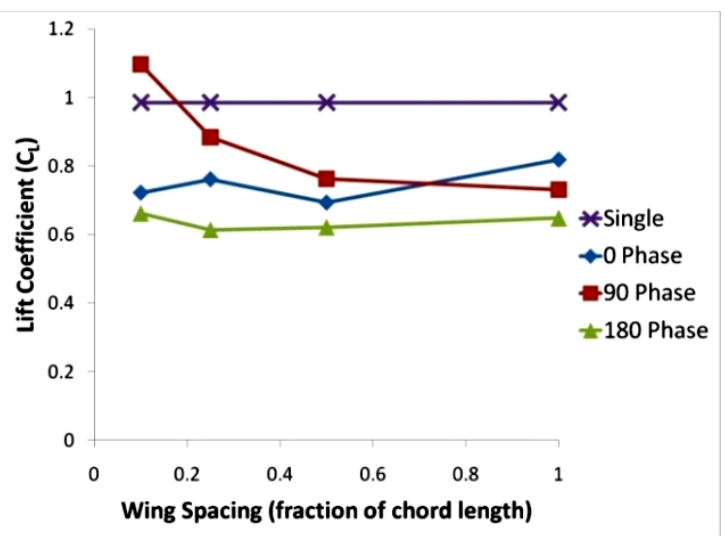

a) Lift

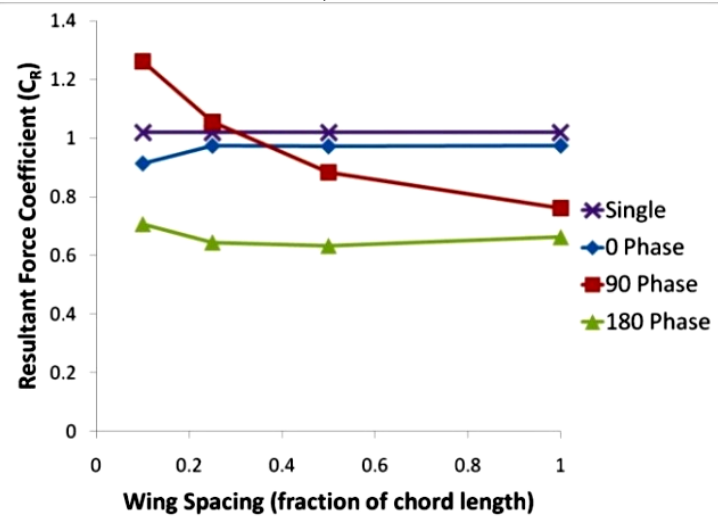

c) Resultant

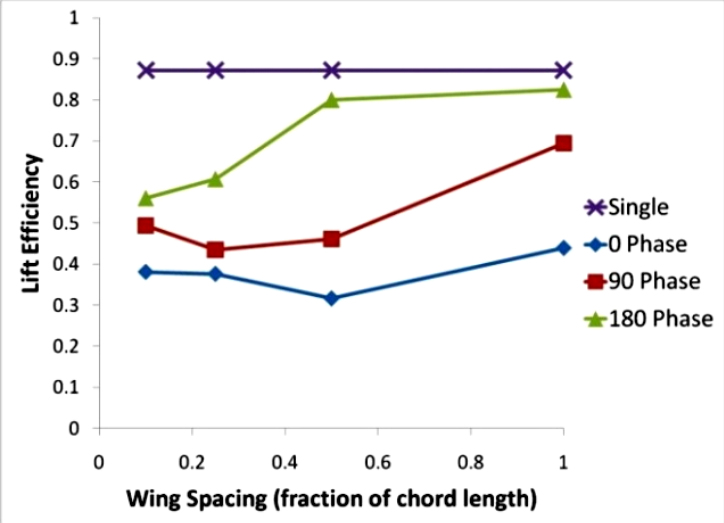

e) Lift Efficiency

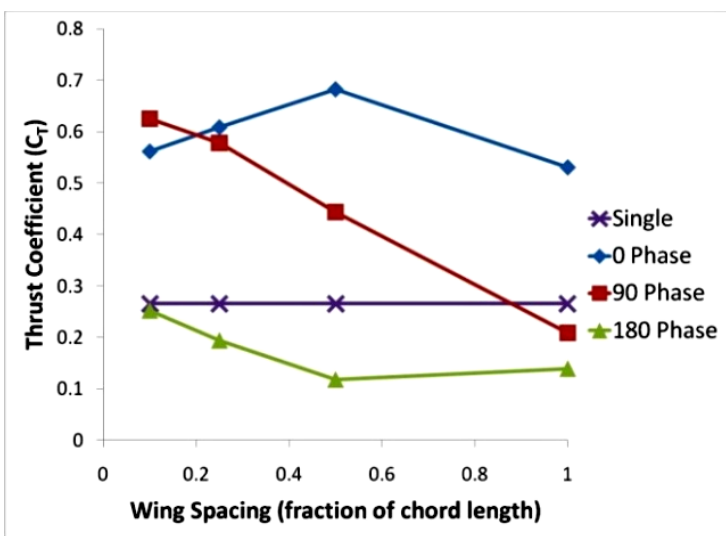

b) Thrust

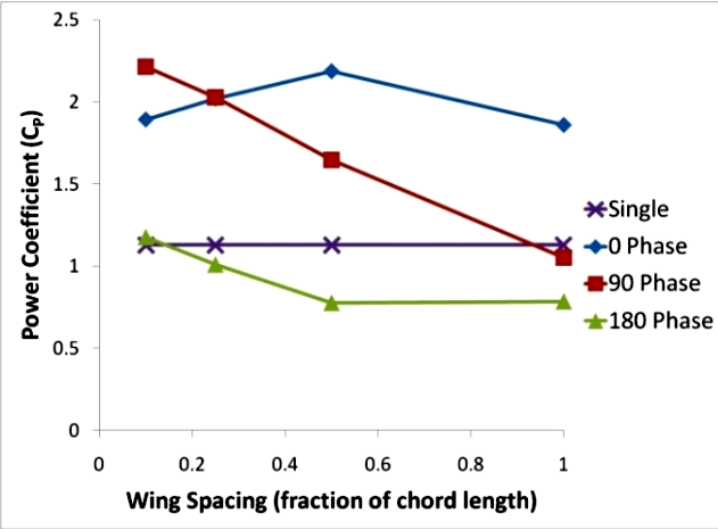

d) Power

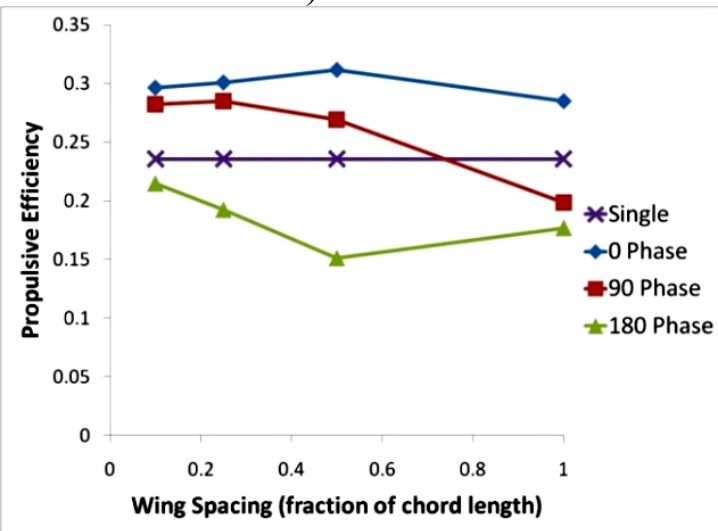

f) Propulsive Efficiency

Figure 28. Cycle averaged lift, thrust, resultant and power coefficients as well as the lift and propulsive efficiency averaged between the fore and hind wings. The results are graphed vs. the spacing for the three phase angles and compared to the single wing.

The averaged results of the fore and hind wings show the same trends observed for the hindwing in Figure 27. At a spacing of $1.0 \mathrm{c}$, the $0^{\circ}$ case produces the largest lift and thrust coefficients while the $180^{\circ}$ case produces the smallest and the $90^{\circ}$ falls 
between the two. As the spacing is decreased, the lift and thrust coefficients increase significantly for the $90^{\circ}$ case until at a spacing of $0.1 \mathrm{c}$, the $90^{\circ}$ produces the most lift and thrust and the $0^{\circ}$ case falls in the middle. In terms of efficiency the $180^{\circ}$ has the highest lift efficiency at all spacings (still below the lift efficiency of a single wing), while the $0^{\circ}$ case has the lowest. The $0^{\circ}$ case has the highest propulsive efficiency at all spacings, while the $180^{\circ}$ case has the lowest. The $0^{\circ}$ case at all four spacings, and the $90^{\circ}$ at the smallest three spacings have a higher propulsive efficiency than the single wing while at all four spacings, the $180^{\circ}$ case has a lower propulsive efficiency than the single wing. 


\section{3D SIMULATION AT RE $=\mathbf{5 0 0 0}$}

A two dimensional analysis is unable to capture three dimensional effects such as tip vortices and the resulting downwash which may have a noticeable influence on fore and hind wing interactions. There is evidence that tip vortices play a role in stabilizing

the LEV on the leading edge ${ }^{[57,58]}$ which could potentially alter the relationship between the hindwing performance and phase angle observed in the two dimensional cases. Furthermore, tip vortices shed from the forewing may interact with tip vortices generated by the hindwing. The final objective of this work is to extend the previous twodimensional study into three-dimensions. To quantify the tip vortex effect, the same pitching and plunging motion used in the $2 \mathrm{D}$ study will be used in the $3 \mathrm{D}$ study. The results from the $3 \mathrm{D}$ model will be compared to the results of its counterpart 2D model that uses the same cross section grid in order to determine what magnitude of effect $3 \mathrm{D}$ flow phenomena have on fore and hind wing interaction. Of specific interest are how tip vortices affect the spanwise LEV formation, whether tip vortex interactions between the fore and hind wings are significant, and whether the fore to hind wing interactions observed in the $2 \mathrm{D}$ case are significantly altered by the $3 \mathrm{D}$ effects. The tandem configuration was simulated at a Reynolds Number of 5000. The spacing between the fore and hind wing used in the study was $0.5 \mathrm{c}$ and the aspect ratio of the $3 \mathrm{D}$ wing was two. 


\section{A. 2D Aerodynamic Force Results}

Figure 29 shows the transient lift and thrust coefficients of the hindwing in the 2D tandem configuration at the three tested phase angles $\left(0^{\circ}, 90^{\circ}, 180^{\circ}\right)$. The coefficients of the standalone single 2D wing with the same kinematics are also shown for comparison purposes. At $\mathrm{t} / \mathrm{T}=0$, all the wings are at the beginning of the downstroke, and at $\mathrm{t} / \mathrm{T}=0.5$ they are at the start of the upstroke.

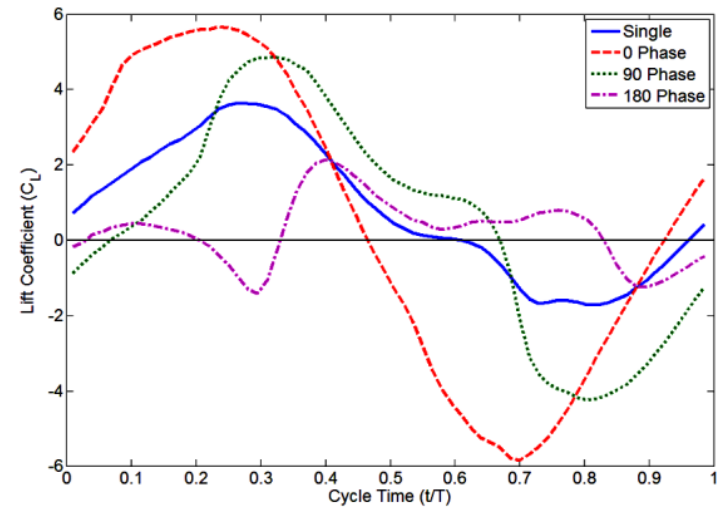

a) Lift

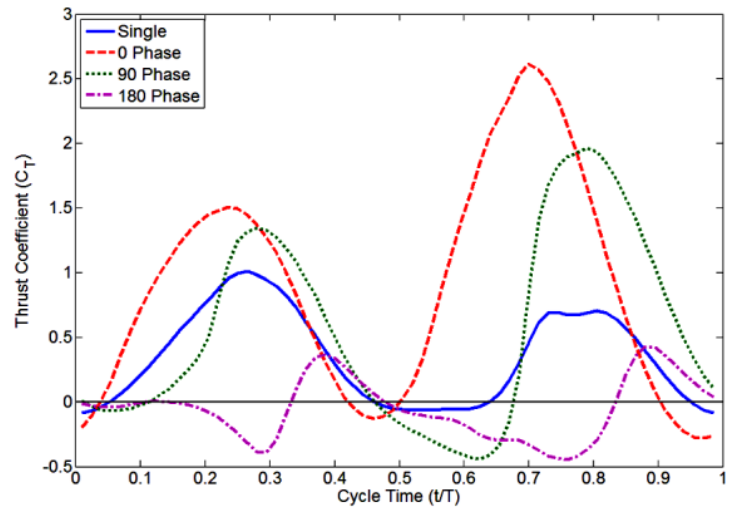

b) Thrust

Figure 29. The two dimensional transient lift and thrust coefficients of the hind wing at the three tested phase angles $\left(0^{\circ}, 90^{\circ}, 180^{\circ}\right)$ and the single wing over one flapping cycle.

It is clear the force coefficients of the hind wing are different from the ones of the single wing, indicating that the forewing has a significant effect on the force production of the hindwing. Furthermore, there are clear differences in the force coefficients among different phase angles, which show that variations in the phase angle between the fore and hind wings significantly alter the wing-wing interaction effect. When flapping in phase $\left(0^{\circ}\right.$ phase $)$, the force histories of the hind wing follow the same trend as the single wing but with much higher amplitude. Shifting the phase angle to $90^{\circ}$ causes a phase shift in the lift and thrust generation as well as a reduction in the lift and thrust amplitudes from the $0^{\circ}$ phase case, though the force amplitudes are still larger compared 
to the single wing. The phase shift in lift at a phase angle of $90^{\circ}$ results in a drastically different lift curve compared to that of the $0^{\circ}$ phase case. At $90^{\circ}$ phase the hindwing produces positive lift through the first half of the upstroke and nearly zero lift through the first half of the downstroke. Another $90^{\circ}$ shift in the phase angle, to a total of $180^{\circ}$, causes a further phase shift in lift and thrust generation and an even greater reduction in the maximum lift and thrust amplitudes generated on the upstroke and downstroke to well below the peak values of the single wing. For this case, the hindwing also shows positive lift generation through a significant portion of the upstroke.

Table 7 shows the cycle averaged lift, thrust, resultant and power coefficients of the single wing and the hindwing at the three tested phase angles $\left(0^{\circ}, 90^{\circ}, 180^{\circ}\right)$. The lift, thrust, and resultant efficiencies are also shown in the table. The most beneficial value in each column is in bold. The power coefficient, $\mathrm{C}_{\mathrm{p}}$, which is a nondimensional measurement of the power required to actuate the wing during a single flapping cycle, was calculated using Equation 18. The lift, propulsive and resultant efficiencies were calculated using Equations 19-21.

\begin{tabular}{|l|c|c|c|c|c|c|c|}
\cline { 2 - 8 } \multicolumn{1}{c|}{} & $\overline{\mathbf{C}_{\mathbf{L}}}$ & $\overline{\mathbf{C}_{\mathbf{L}}}$ Eff. & $\overline{\mathbf{C}_{\mathbf{T}}}$ & $\overline{\mathbf{C}_{\mathbf{T}}}$ Eff. & $\overline{\mathbf{C}_{\mathbf{R}}}$ & $\overline{\mathbf{C}_{\mathbf{R}}}$ Eff. & Power \\
\hline Single & $\mathbf{0 . 8 0 3}$ & $\mathbf{5 8 . 1 \%}$ & 0.343 & $24.8 \%$ & 0.873 & $\mathbf{6 3 . 1 \%}$ & 1.383 \\
\hline 0 Hind & 0.423 & $15.9 \%$ & $\mathbf{0 . 8 8 5}$ & $\mathbf{3 3 . 2 \%}$ & $\mathbf{0 . 9 8 1}$ & $36.8 \%$ & 2.667 \\
\hline 90 Hind & 0.402 & $18.6 \%$ & 0.547 & $25.3 \%$ & 0.679 & $31.5 \%$ & 2.158 \\
\hline 180 Hind & 0.254 & $50.6 \%$ & -0.052 & $-10.4 \%$ & 0.259 & $51.6 \%$ & $\mathbf{0 . 5 0 2}$ \\
\hline
\end{tabular}

Table 7. Two dimensional cycle averaged values for the hindwings compared to a single wing.

Table 7 shows that the single wing has the highest lift coefficient, lift efficiency and resultant force efficiency for the tested cases. The hind wing at a phase angle of $0^{\circ}$ has the highest thrust and resultant force coefficients as well as the highest propulsive efficiency. Switching to a phase angle of $180^{\circ}$, the hindwing has the lowest power 
consumption out of all the tested cases. At a phase angle of $90^{\circ}$, the hindwing shows performance between that of the $0^{\circ}$ and $180^{\circ}$ cases.

Table 8 shows the results for the tandem configuration by averaging the lift, thrust, resultant force and power coefficient between the fore and hind wing. Again, the most desirable result in each column is bolded.

\begin{tabular}{|l|c|c|c|c|c|c|c|}
\cline { 2 - 8 } \multicolumn{1}{c|}{} & $\overline{\mathbf{C}_{\mathbf{L}}}$ & $\overline{\mathbf{C}_{\mathbf{L}}}$ Eff. & $\overline{\mathbf{C}_{\mathbf{T}}}$ & $\overline{\mathbf{C}_{\mathbf{T}}}$ Eff. & $\overline{\mathbf{C}_{\mathbf{R}}}$ & $\overline{\mathbf{C}_{\mathbf{R}}}$ Eff. & Power \\
\hline Single & 0.678 & $62.2 \%$ & 0.291 & $26.7 \%$ & 0.738 & $67.7 \%$ & 1.090 \\
\hline 0 Phase & $\mathbf{0 . 7 3 2}$ & $33.7 \%$ & $\mathbf{0 . 6 9 5}$ & $\mathbf{3 2 . 0 \%}$ & $\mathbf{1 . 0 6 9}$ & $49.2 \%$ & 2.173 \\
\hline 90 Phase & 0.726 & $40.3 \%$ & 0.480 & $26.7 \%$ & 0.904 & $50.2 \%$ & 1.800 \\
\hline 180 Phase & 0.594 & $\mathbf{6 6 . 8 \%}$ & 0.157 & $17.7 \%$ & 0.631 & $\mathbf{7 1 . 0 \%}$ & $\mathbf{0 . 8 8 8}$ \\
\hline
\end{tabular}

Table 8. Two dimensional cycle averaged values averaged between the fore and hind wing for each phase angle compared to a single wing.

The results in Table 8 show that the tandem wing configuration can outperform the single wing in each category by altering the phase angle between the fore and hind wings. With a phase angle of $0^{\circ}$, the tandem configuration produces the largest cycle averaged forces and has the highest propulsive efficiency. At a phase angle of $180^{\circ}$ the tandem configuration has the highest lift and resultant force efficiencies and the lowest power consumption. The $90^{\circ}$ case falls between the performance of the $0^{\circ}$ and $180^{\circ}$ cases. The ability to change the phasing between the fore and hind wings allows insects utilizing the tandem configuration to change their flight mode to obtain the desired performance, from high powered, high force generation at $0^{\circ}$, to low powered, high efficiency at $180^{\circ}$, to a compromise between the two at $90^{\circ}$.

\section{B. 2D Vorticity Contours}

The relationship between the lift and thrust production of the hind wing and the phase angle shows a strong correlation with the effect of the forewing on the hind wing LEVs. Figure 30-Figure 32 show the snapshots of vorticity contours at different time 
instants for the $0^{\circ}, 90^{\circ}$ and $180^{\circ}$ phase cases, respectively. The vorticity contours of the single wing are shown alongside each case for the sake of comparison.

The first row in Figure 30 show the single wing and $0^{\circ}$ phase case early in the downstroke. At this point, the hindwing passes through the vortex shed from the forewing, which interacts with the LEV that is forming on the top of the hindwing. The vortex from the forewing and the LEV from the hindwing both rotate clockwise (CW). This interaction reinforces the LEV generated by the hindwing, increasing its size, as shown in Figure 30d, which shows the hindwing at $\mathrm{t} / \mathrm{T}=0.25$ when lift and thrust reach their peak. This interaction is repeated on the upstroke and the resulting LEV is shown shedding from the bottom of the hindwing in Figure 30b. The larger LEV results in the increase in lift and thrust generated on the downstroke and upstroke, as evidenced in Figure 29.

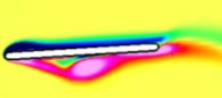

a) Single, $t / \mathbf{T}=\mathbf{0 . 0 7}$

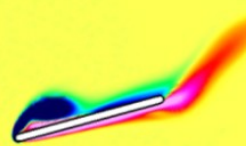

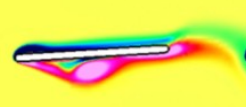
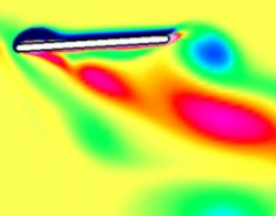

b) Tandem wing 0 Phase, $t / T=0.07$

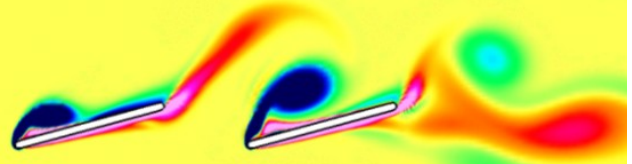

d) Tandem wing 0 Phase, $t / T=0.25$
20.00

16.00

12.00

8.00

$-4.00$

0.00

$-4.00$

$-8.00$

$-12.00$

$-16.00$

$-20.00$

c) Single, $t / T=0.25$

Figure 30. The vorticity contours illustrating the vortex interactions between the fore and hind wing with a phase angle of $0^{\circ}$. At this phase angle the vortex interactions are constructive and increase the size of the LEV generated by the hindwing. 


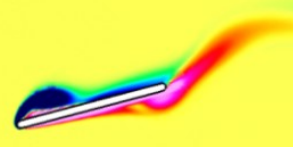

a) Single, $t / T=0.22$

c) Single, $t / T=0.40$

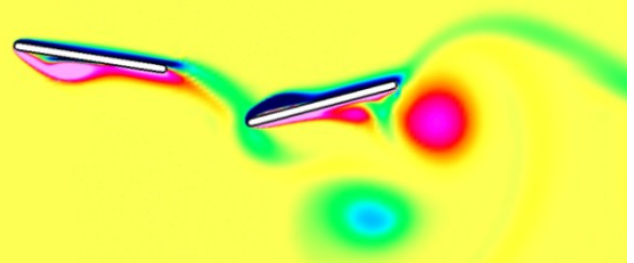

b) Tandem wing 90 Phase, $t / T=0.22$
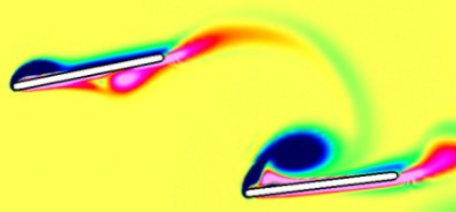

d) Tandem wing 90 Phase, $t / T=0.40$

20.00

16.00

12.00

8.00

4.00

$-0.00$

$-4.00$

$-8.00$

$-12.00$

$-16.00$

$-20.00$

Figure 31. The vorticity contours illustrating the vortex interactions between the fore and hind wing with a phase angle of $90^{\circ}$. At this phase angle the vortex interactions cause an LEV to remain attached to the top of the hindwing through the first half of the upstroke.

The vortex interaction of the $90^{\circ}$ phase case (Figure 31 ) is similar to the $0^{\circ}$ phase case in some aspects. During the downstroke, the hindwing of the $90^{\circ}$ case also passes through the $\mathrm{CW}$ vortex shed from the forewing which interacts with the CW LEV generated on the top of the hindwing; however, this interaction occurs later in the downstroke $(\mathrm{t} / \mathrm{T}=0.22)$ than the $0^{\circ}$ case. This causes a phase shift in the LEV generation of the hindwing. The interaction in the second half of the downstroke reinforces the $\mathrm{CW}$ LEV formation on the top of the hindwing, delays it shedding, and allows it to persist through the early portion of the upstroke. The interaction is then repeated during the second half of the upstroke, which reinforces the counterclockwise (CCW) LEV forming on the bottom of the hindwing which delays its shedding and allows it to remain attached through the first half of the downstroke, as shown in Figure 31b. By keeping the LEV attached to the top of the airfoil through the first half of the upstroke, the hindwing generates positive lift during that portion of the cycle. 


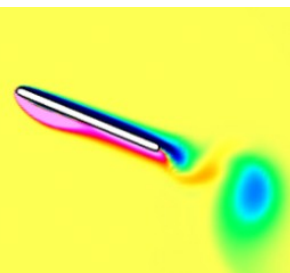

a) Single, $t / T=0.84$

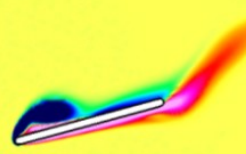

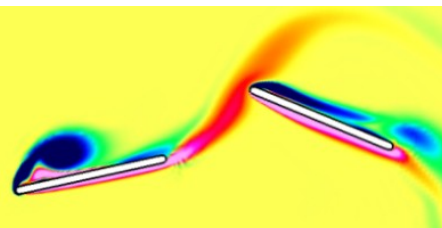

b) Tandem wing 180 Phase, $t / T=0.84$
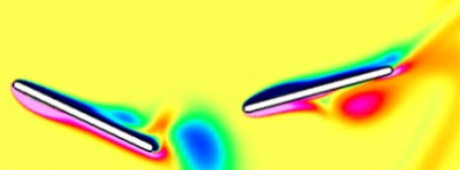

c) Single, $50 \% \mathrm{t} / \mathrm{T}=\mathbf{0 . 2 5}$

d) Tandem wing 180 Phase, $t / T=0.25$

Figure 32. The vorticity contours illustrating the vortex interactions between the fore and hind wing with a phase angle of $180^{\circ}$. At this phase angle the vortex interactions are destructive and dampen the size of the LEV generated by the hindwing.

The vortex interactions that occur for the $180^{\circ}$ phase case (Figure 32) are nearly the opposite of the $0^{\circ}$ phase case. At the start of the stroke reversal, the hindwing of the $180^{\circ}$ case passes through the $\mathrm{CCW}$ vortex shed from the forewing. This dampens out the CW LEV that would normally form on the top of the hindwing during the downstroke and instead a small CCW LEV is generated on the bottom, which can be seen in Figure $32 \mathrm{~d}$. This interaction is then repeated during the upstroke, which dampens out the CCW LEV that would normally form on the bottom of the hindwing. The hindwing instead generates a small CW LEV on the top, which is shown in Figure 32b. The resulting weak LEV formation leads to the weak lift and thrust production observed in Figure 29.

In general, the vortex interactions between the fore and hind wings can be characterized as either constructive or destructive. When the hindwing flaps in phase $\left(0^{\circ}\right.$ phase lag), constructive vortex interactions take place which reinforce the LEV generation of the hindwing, leading to large LEVs and the resulting increase in the peak lift and thrust. Conversely, the $180^{\circ}$ case exhibits destructive vortex interaction which 
dampens out LEV generation. This leads to weak LEV generation resulting in the weak lift and thrust production. The $90^{\circ}$ case falls in between the $0^{\circ}$ and $180^{\circ}$ cases, where the vortex interactions cause a phase shift in LEV production, resulting in a phase shift in the lift and thrust production. Furthermore, the vortex interactions of the $180^{\circ}$ case result in the hindwing producing a significant amount of positive lift during the upstroke and negative lift during the downstroke. This results in the significant power consumption reduction for the $180^{\circ}$ case due to the direction of force production being coincident to the direction of wing motion for a significant portion of the flapping cycle.

\section{3D Aerodynamic Force Results}

Figure 33 shows the transient lift and thrust coefficients of the hind wing in the 3D tandem configuration at the three tested phase angles $\left(0^{\circ}, 90^{\circ}, 180^{\circ}\right)$ and a single wing over one flapping cycle. At $\mathrm{t} / \mathrm{T}=0$, all the wings are at the beginning of the downstroke, and at $\mathrm{t} / \mathrm{T}=0.5$ they are at the start of the upstroke.

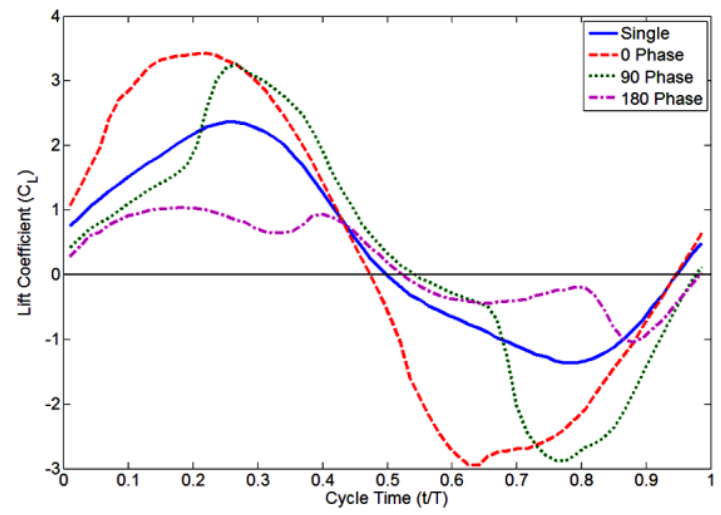

a) Lift

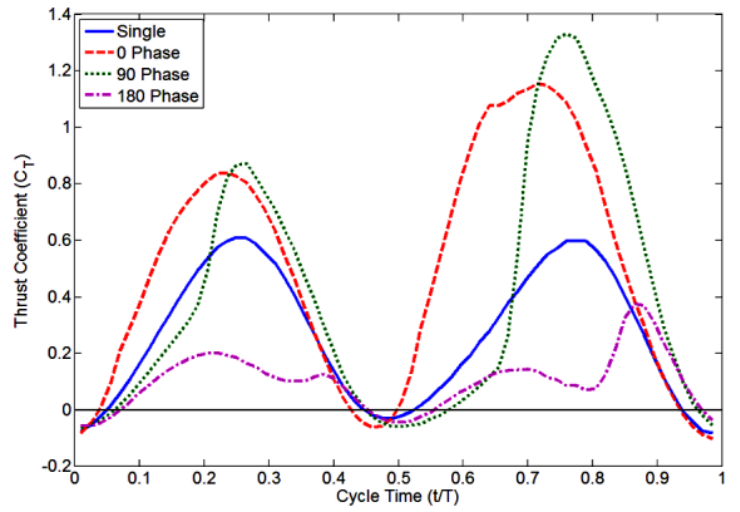

b) Thrust

Figure 33. The transient lift and thrust coefficients of the 3D hind wing at the three tested phase angles $\left(0^{\circ}, 90^{\circ}, 180^{\circ}\right)$ and the single wing over one flapping cycle.

The relationship between the phase angle and the lift and thrust production of the hindwing is very similar to that of the $2 \mathrm{D}$ case shown in Figure 29. For the $0^{\circ}$ phase lag case the hindwing shows significantly higher peak lift and thrust during the upstroke and 
downstroke. For the $90^{\circ}$ phase lag case the hindwing exhibits a slight phase lag in the lift and thrust with similar magnitudes of peak lift and thrust to the single wing. The $180^{\circ}$ hindwing shows a phase lag in the lift and thrust with significantly lower magnitudes of peak lift and thrust than those of the single wing. The differences in the two dimensional and three dimensional transient force results are displayed more clearly in Figure 34Figure 37 which plot the 2D and 3D lift and thrust together for the single wing and the tandem hindwing at the three tested phase angles. The main difference between the $2 \mathrm{D}$ and $3 \mathrm{D}$ force results is that all of the 3D cases show a reduction in the lift and thrust peak magnitude compared to the two dimensional results. This reduction is mostly the result of tip vortices generated by a finite aspect ratio wing.

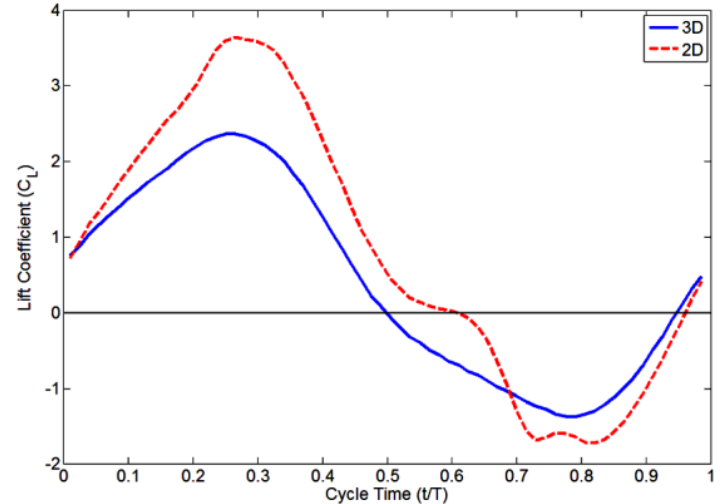

a) Lift

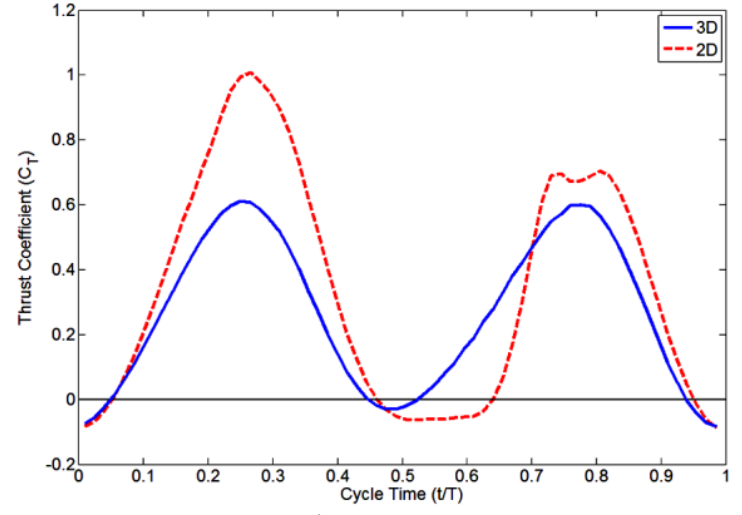

b) Thrust

Figure 34. The transient lift and thrust coefficients of the 3D single wing compared to the same results for the $2 \mathrm{D}$ case. 


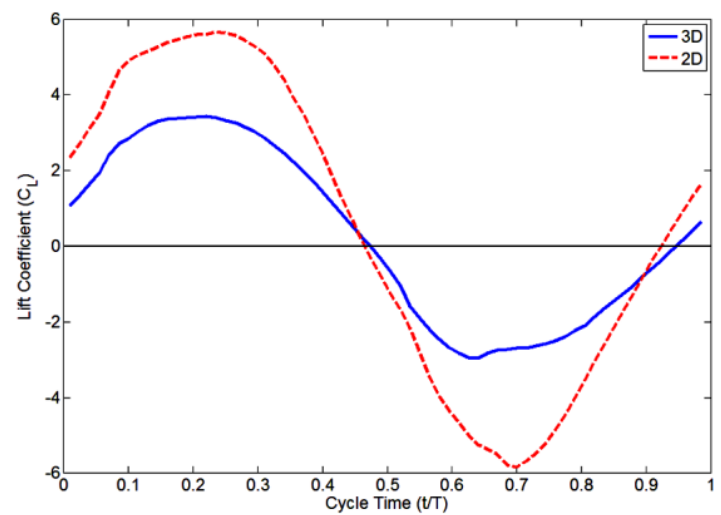

a) Lift

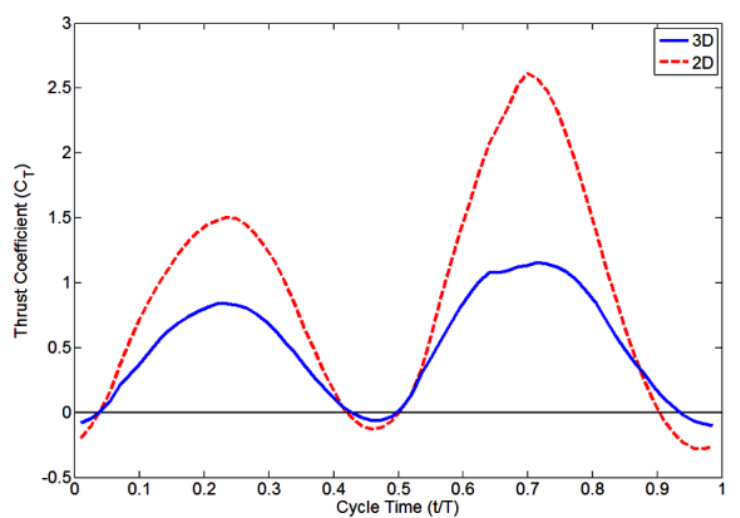

b) Thrust

Figure 35. The transient lift and thrust coefficients of the $3 D$ hindwing at $0^{\circ}$ compared to the same results for the $2 \mathrm{D}$ case.

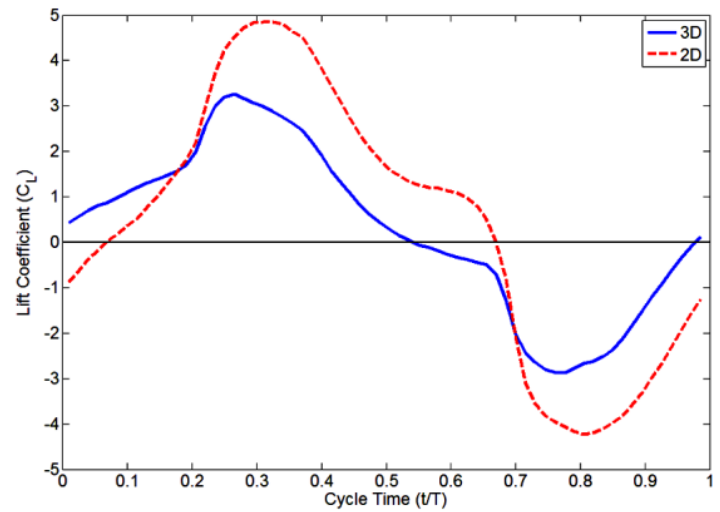

a) Lift

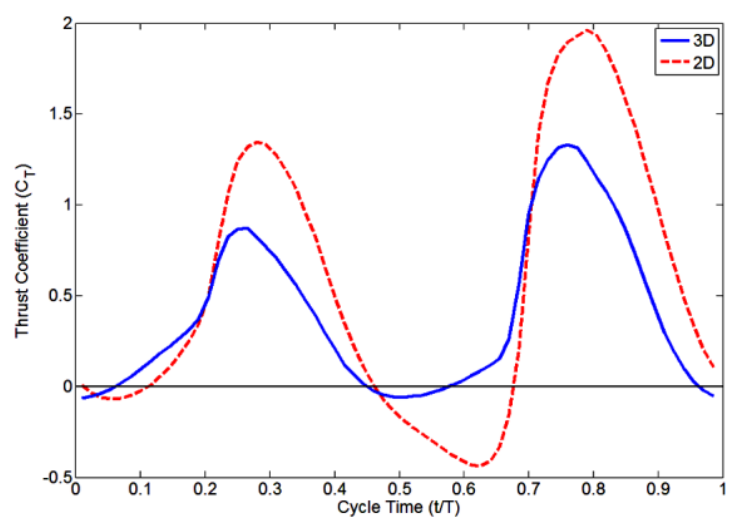

b) Thrust

Figure 36. The transient lift and thrust coefficients of the $3 \mathrm{D}$ hindwing at $90^{\circ}$ compared to the same results for the $2 \mathrm{D}$ case.

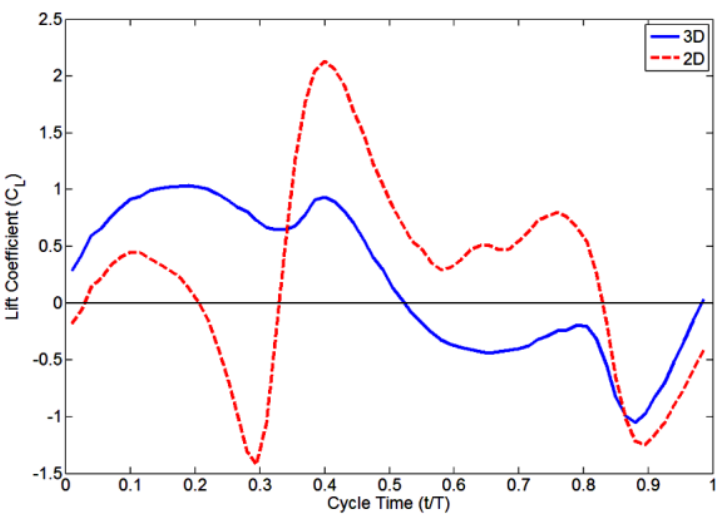

a) Lift

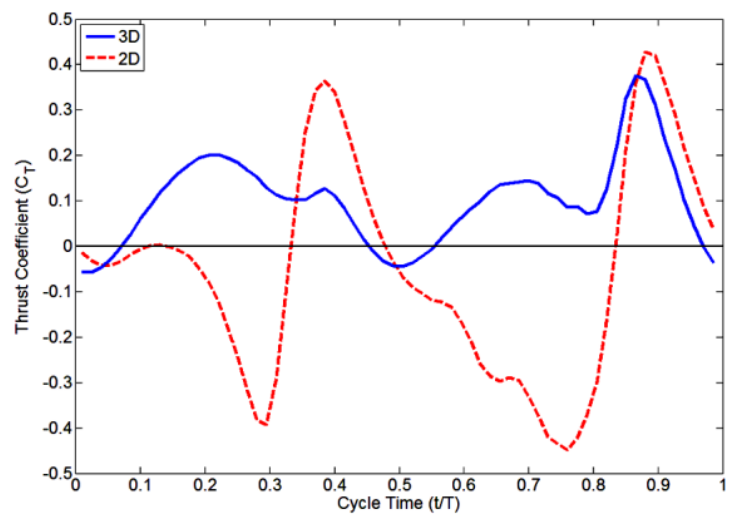

b) Thrust

Figure 37. The transient lift and thrust coefficients of the $3 D$ hindwing at $180^{\circ}$ compared to the same results for the $2 \mathrm{D}$ case.

Table 9 compares the cycle averaged parameters between the hindwing and a standalone single wing. The most beneficial value in each column is in bold. 


\begin{tabular}{|c|c|c|c|c|c|c|c|}
\cline { 2 - 8 } \multicolumn{1}{c|}{} & $\overline{\mathbf{C}_{\mathbf{L}}}$ & $\overline{\mathbf{C}_{\mathbf{L}}}$ Eff. & $\overline{\mathbf{C}_{\mathbf{T}}}$ & $\overline{\mathbf{C}_{\mathbf{T}}}$ Eff. & $\overline{\mathbf{C}_{\mathbf{R}}}$ & $\overline{\mathbf{C}_{\mathbf{R}}}$ Eff. & Power \\
\hline Single & $\mathbf{0 . 3 5 9}$ & $\mathbf{3 7 . 2 \%}$ & 0.264 & $27.3 \%$ & 0.446 & $\mathbf{4 6 . 1 \%}$ & 0.966 \\
\hline 0 Hind & 0.219 & $14.4 \%$ & $\mathbf{0 . 4 9 4}$ & $\mathbf{3 2 . 4 \%}$ & $\mathbf{0 . 5 4 0}$ & $35.5 \%$ & 1.524 \\
\hline 90 Hind & 0.215 & $16.0 \%$ & 0.391 & $29.1 \%$ & 0.446 & $33.2 \%$ & 1.343 \\
\hline 180 Hind & 0.15 & $29.0 \%$ & 0.099 & $19.1 \%$ & 0.180 & $34.7 \%$ & $\mathbf{0 . 5 1 8}$ \\
\hline
\end{tabular}

Table 9. Three dimensional cycle averaged values for the hindwings compared to a single wing.

The three dimensional results in Table 9 show a similar relationship between the phase angle and the cycle averaged results of the hindwing as the two dimensional results shown in Table 7 . At $0^{\circ}$ phase lag the hindwing generates the highest thrust and resultant coefficients as well as the highest propulsive efficiency, while switching to $180^{\circ}$ requires the lowest power coefficient and also increases the lift efficiency. The three dimensional results; however, indicate that at $90^{\circ}$ phase lag, the hindwing has the highest resultant efficiency among the three tested phase angles, while the $180^{\circ}$ hindwing has the lowest resultant efficiency; whereas, the two dimensional results showed that the $180^{\circ}$ hindwing had the highest resultant efficiency. Furthermore, while the two and three dimensional results both show that the single wing has a higher lift coefficient than any of the hindwings, the three dimensional results also show that the single wing has the highest lift and resultant efficiencies; whereas, the two dimensional results indicated that the $180^{\circ}$ hindwing had the highest lift and resultant efficiencies.

Table 10 shows the results for the tandem configuration by averaging the values between the fore and hind wing. Again, the most desirable result in each column is bolded. 


\begin{tabular}{|l|c|c|c|c|c|c|c|}
\cline { 2 - 8 } \multicolumn{1}{c|}{} & $\overline{\mathbf{C}_{\mathbf{L}}}$ & $\overline{\mathbf{C}_{\mathbf{L}}}$ Eff. & $\overline{\mathbf{C}_{\mathbf{T}}}$ & $\overline{\mathbf{C}_{\mathbf{T}}}$ Eff. & $\overline{\mathbf{C}_{\mathbf{R}}}$ & $\overline{\mathbf{C}_{\mathbf{R}}}$ Eff. & Power \\
\hline Single & $\mathbf{0 . 3 5 9}$ & $37.2 \%$ & 0.264 & $27.3 \%$ & 0.446 & $46.1 \%$ & 0.966 \\
\hline 0 Phase & 0.308 & $24.7 \%$ & $\mathbf{0 . 3 9 9}$ & $\mathbf{3 2 . 0 \%}$ & $\mathbf{0 . 5 2 0}$ & $41.7 \%$ & 1.247 \\
\hline 90 Phase & 0.314 & $28.3 \%$ & 0.330 & $29.8 \%$ & 0.469 & $42.4 \%$ & 1.107 \\
\hline 1 80 Phase & 0.261 & $\mathbf{3 8 . 6 \%}$ & 0.179 & $26.5 \%$ & 0.317 & $\mathbf{4 6 . 8 \%}$ & $\mathbf{0 . 6 7 7}$ \\
\hline
\end{tabular}

Table 10. Three dimensional cycle averaged values averaged between the fore and hind wing for each phase angle compared to a single wing.

The same trends shown in Table 9 for the hindwings individually are mirrored in Table 10 for the tandem configuration as a whole. Like the results in Table 9, the three dimensional results in Table 10 show a similar relationship between the cycle averaged values and the phase angle. Unlike the two dimensional results; however, the three dimensional results do not show higher performance of the tandem wing than the single wing for all values. Instead, the three dimensional results show that the single wing has a higher lift coefficient and higher lift and resultant efficiencies than the tandem configuration. Also, in general, the three dimensional results in Table 9 and Table 10 exhibit lower force coefficients and efficiencies than the two dimensional results in Table 7 and Table 8.

\section{3D Vorticity Contours}

The iso-surface vorticity contours for the three dimensional cases are presented in Figure 38-Figure 40. To ensure a fair comparison with the $2 \mathrm{D}$ results, only the component in the spanwise direction is shown. The iso-surface contours of the $3 \mathrm{D}$ single wing are shown alongside the tandem wing for comparison. 

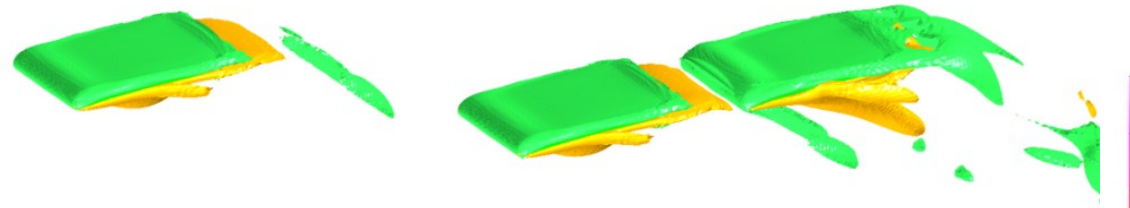

20.00

16.00

12.00

8.00

a) Single, $t / \mathbf{T}=\mathbf{0 . 0 7}$

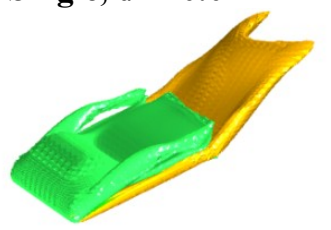

b) Tandem wing 0 Phase, $t / T=0.07$

4.00

$-0.00$

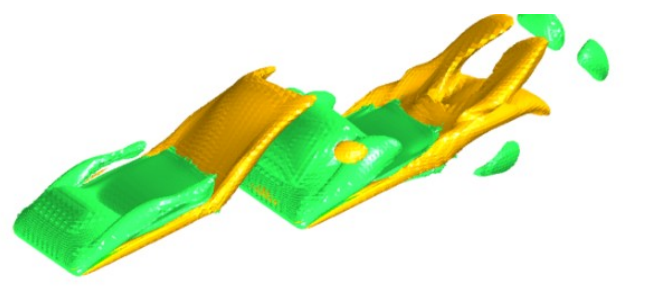

$-4.00$

$-8.00$

$-12.00$

$-16.00$

c) Single, $t / \mathbf{T}=\mathbf{0 . 2 5}$

d) Tandem wing 0 Phase, $t / T=0.25$

Figure 38. The iso-surface contours of the vorticity in the spanwise direction, illustrating the vortex interactions between the fore and hind wing with a phase angle of $0^{\circ}$. The three dimensional iso-surfaces show the same constructive interaction observed in the 2D case.

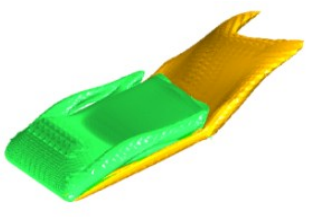

a) Single, $\mathbf{t} / \mathbf{T}=\mathbf{0 . 2 2}$

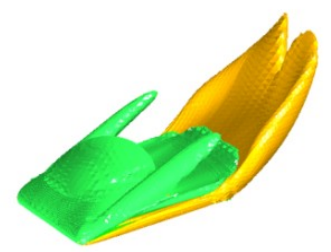

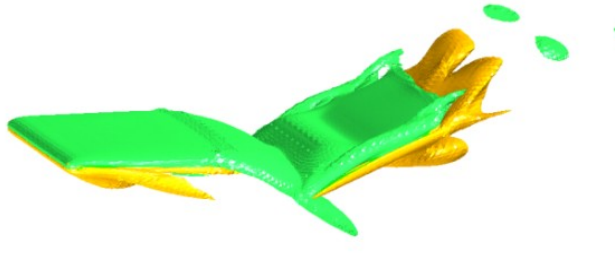

b) Tandem wing 90 Phase, $t / T=0.22$

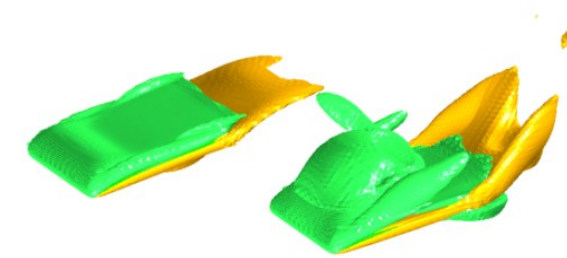

d) Tandem wing 90 Phase, $t / T=0.40$
20.00
16.00
12.00
8.00
4.00
0.00
$-4.00$
$-8.00$
$-12.00$
$-16.00$
$-20.00$

c) Single, $t / \mathbf{T}=\mathbf{0 . 4 0}$

Figure 39. The iso-surface contours of the vorticity in the spanwise direction, illustrating the vortex interactions between the fore and hind wing with a phase angle of $90^{\circ}$. 


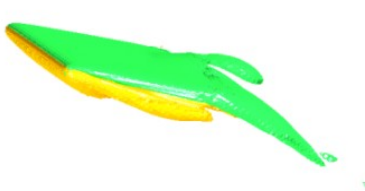

a) Single, $t / T=0.84$

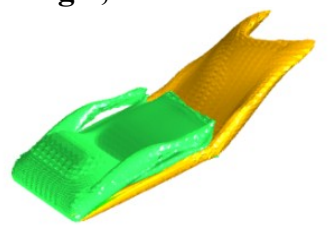

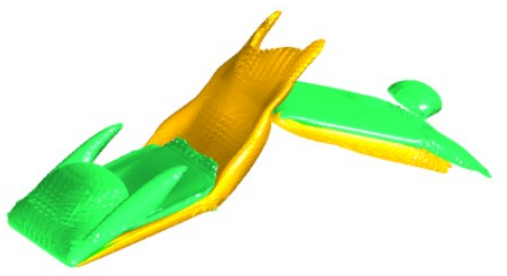

b) Tandem wing 180 Phase, $t / T=0.84$

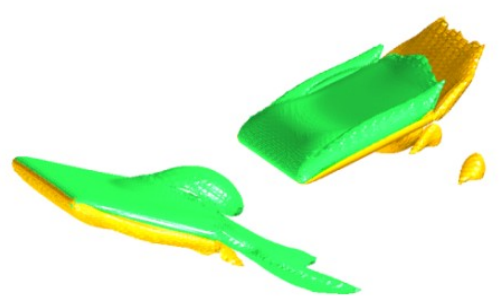

d) Tandem wing 180 Phase, $t / T=0.25$
20.00

16.00

12.00

8.00

4.00

$-0.00$

$-4.00$

$-8.00$

$-12.00$

$-16.00$

$-20.00$

c) Single, $t / T=0.25$

Figure 40. The three dimensional iso-surface contours of the vorticity component in the spanwise direction, illustrating the vortex interactions between the fore and hind wing with a phase angle of $180^{\circ}$. The three dimensional iso-surfaces show the same destructive interaction observed in the $2 \mathrm{D}$ case.

The vortex interactions shown in the $3 \mathrm{D}$ cases are very similar to those in the $2 \mathrm{D}$ cases. In the $0^{\circ}$ phase lag case, the vortex shed from the forewing strengthens the same sign LEV on the hind wing; in the $180^{\circ}$ case, the vortex from the forewing dampens the opposite sign LEV on the hind wing.

\section{E. Tip Vortices and Wing Interaction}

Figure 41-Figure 43 shows the interaction between tip vortices from the forewing and hindwing by plotting the iso-surfaces of the vorticity about the streamwise axis. The tandem wing tip vortices are presented alongside the tip vortices of the single wing for comparison. Iso-surface contours of the vorticies are shown at different time instants of the flapping cycle. 

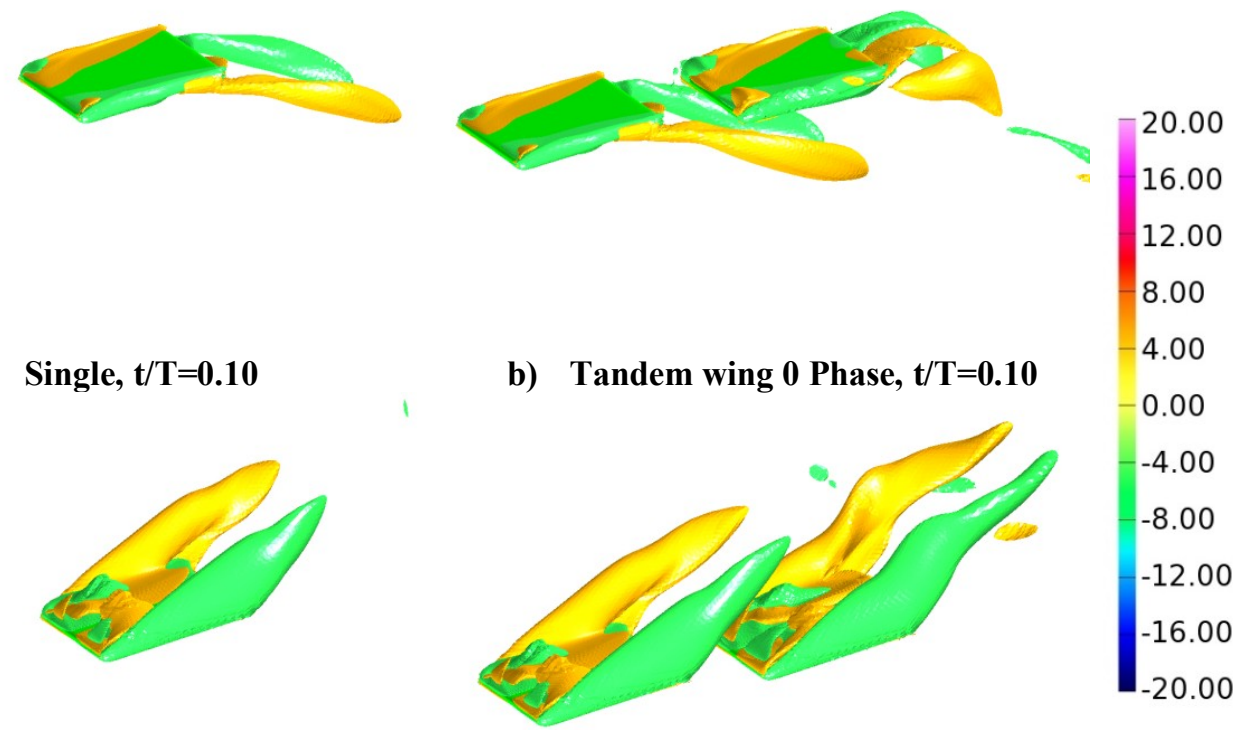

c) Single, $\mathbf{t} / \mathbf{T}=\mathbf{0 . 4 0}$

d) Tandem wing 0 Phase, $t / T=0.40$

Figure 41. The tip vortex interaction between the fore and hind wings at $0^{\circ}$ phase lag. In this case the hindwing bisects tip vortices shed by the forewing on both the downstroke and upstroke.

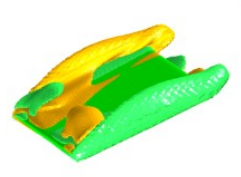

a) Single, $\mathbf{t} / \mathbf{T}=\mathbf{0 . 2 2}$

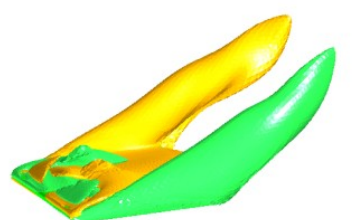

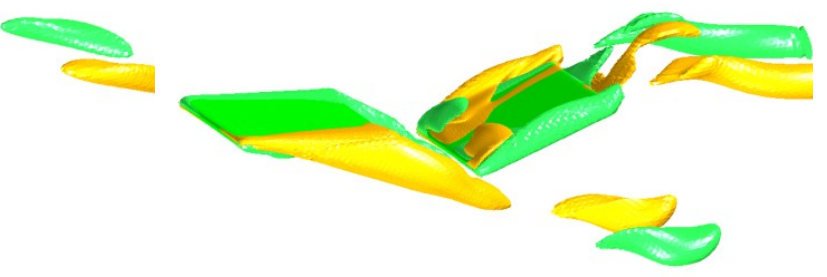

b) Tandem wing 90 Phase, $t / T=0.22$

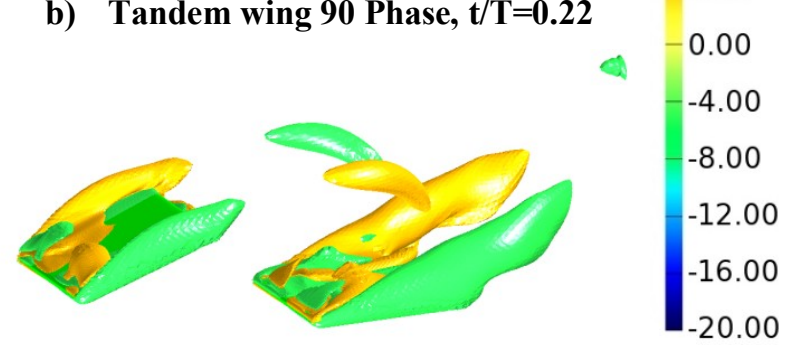

c) Single, $\mathrm{t} / \mathrm{T}=\mathbf{0 . 4 9}$

d) Tandem wing 90 Phase, $t / T=0.49$

Figure 42. The tip vortex interaction between the fore and hind wings at $90^{\circ}$ phase lag. Like the $0^{\circ}$ case, the hindwing bisects the tip vortices shed by the forewing during both the downstroke and upstroke. 


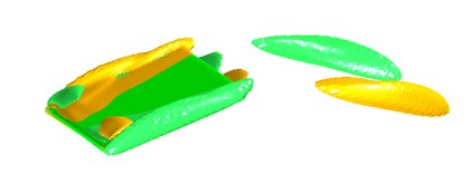

a) Single, $t / T=0.16$

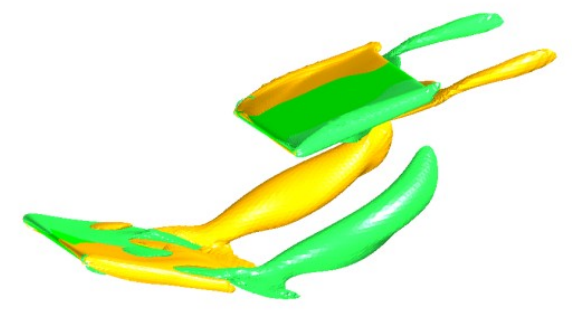

20.00

16.00

12.00

8.00

4.00

0.00

$-4.00$

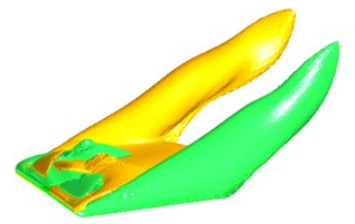

b) Tandem wing 180 Phase, $t / T=0.16$

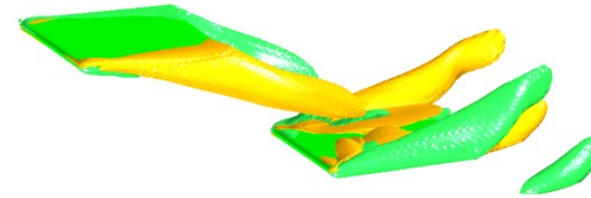

$-8.00$

$-12.00$

$-16.00$

$-20.00$

c) Single, $t / T=0.49$

d) Tandem wing 180 Phase, t/T=0.49

Figure 43. The tip vortex interaction between the fore and hind wings at $180^{\circ}$ phase lag. In this case there is no noticeable interaction between tip vortices shed by the forewing with the hindwing.

For the most part, the tip vortex generation of the hindwing only shows minor variation with phase angle. The timing of the tip vortex formation and shedding is independent of phase angle. The hindwing always starts to form the tip vortex at the start of a stroke and sheds the tip vortex at the end of a stroke. There are, however; interactions that occur between the tip vortices shed by the forewing and those generated by the hindwing which vary with phase angle. At $0^{\circ}$ phase lag, on both the upstroke and downstroke, the hindwing bisects the tip vortices shed from the forewing, which interact with opposite signed tip vortices generated by the hindwing. The interaction on the downstroke is shown in Figure 41b. Despite this interaction, the tip vortices shed by the hindwing (Figure 41d) are slightly larger than those generated by a single wing. For the $180^{\circ}$ case, the tip vortices shed by the forewing pass both above and below the hindwing without any interaction (Figure 43b). Even without interaction, the tip vortices shed by 
the hindwing are slightly weaker than those of the single wing. Finally, the hindwing of the $90^{\circ}$ passes through tip vortices shed by the forewing during both the upstroke and downstroke which interact with the opposite signed tip vortices generated by the hindwing (Figure $42 \mathrm{~b}, \mathrm{~d}$ ). The tip vortices shed by the hindwing in this case, however, don't show a significant difference compared to the tip vortices of the single wing. Ultimately, the effect of these different tip vortex interactions, however; is minimal as the tip vortices are confined to the very tip of the wing.

To understand the interactions between the tip vortices and LEV, we show the LEV at three different positions along the span $(50 \%, 75 \%, 95 \%)$. Results from both single wing and hindwing are shown. The vorticity contours are shown during the downstroke for each case, at the time instant where the case displays peak force production $\left(\mathrm{t} / \mathrm{T}=0.25\right.$ for the single wing and the hindwing at $90^{\circ}$ phase lag angle, $\mathrm{t} / \mathrm{T}=0.2$ for the hindwing at $0^{\circ}$ phase lag angle and $\mathrm{t} / \mathrm{T}=0.175$ for the hindwing at $180^{\circ}$ phase lag angle).

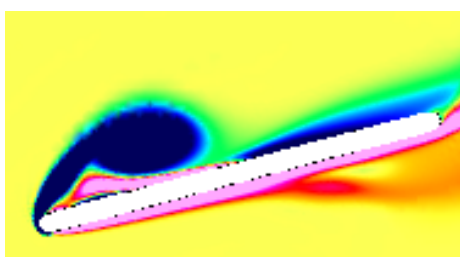

a) $\mathbf{5 0 \%}$ span

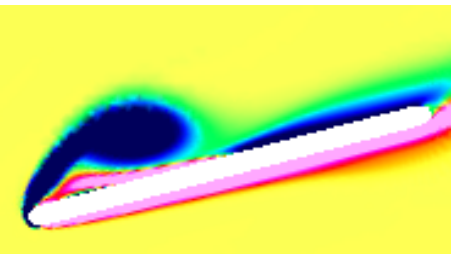

b) $75 \%$ span

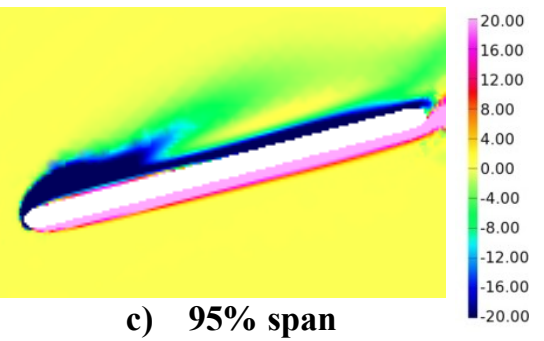

c) $95 \%$ span

Figure 44.Spanwise variation of the $L E V$ of the single wing at $t / T=0.25$.

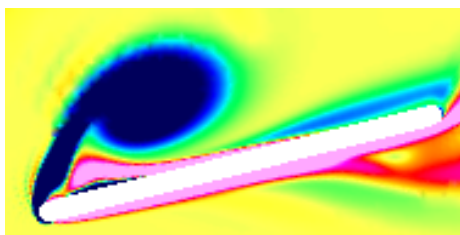

a) $\mathbf{5 0 \%}$ span

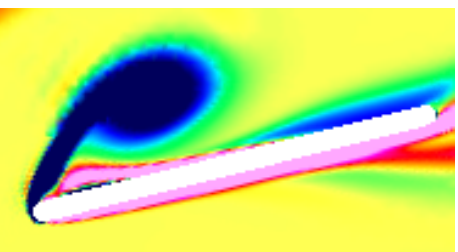

b) $75 \%$ span

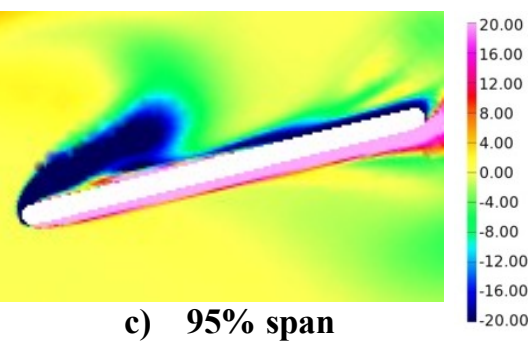

c) $95 \%$ span

.00
.00
.00
.00
.00
.00
.00
00
2.00
6.00
.00
Figure 45. Spanwise variation of the LEV of the hindwing at $0^{\circ}$ phase lag angle and $\mathbf{t} / \mathbf{T}=\mathbf{0 . 2}$. 


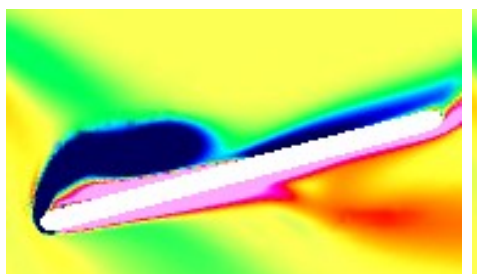

a) $\mathbf{5 0 \%}$ span

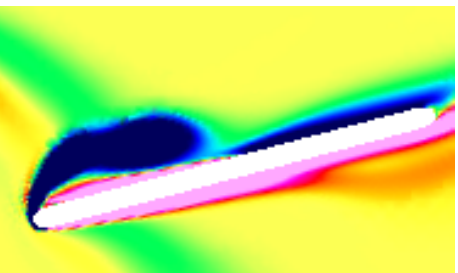

b) $75 \%$ span

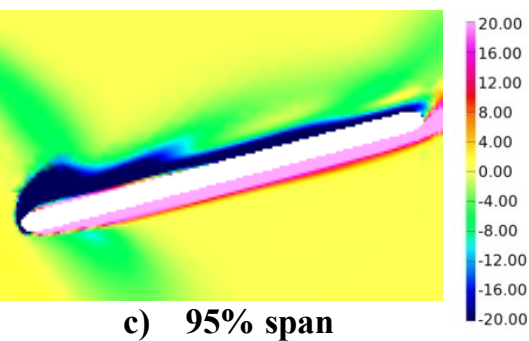

c) $95 \% \operatorname{span}$

Figure 46. Spanwise variation of the LEV of the hindwing at $90^{\circ}$ phase lag angle and $\mathbf{t} / \mathbf{T}=\mathbf{0 . 2 5}$.

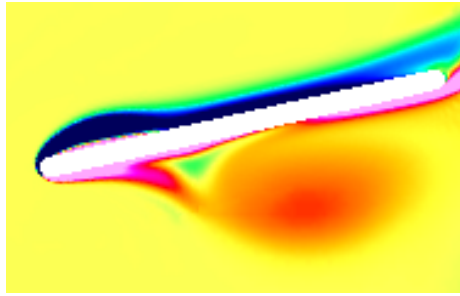

a) $50 \%$ span

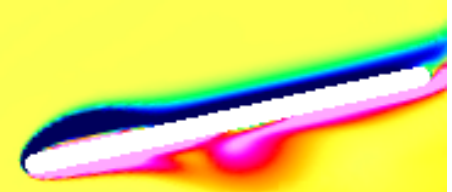

b) $75 \%$ span

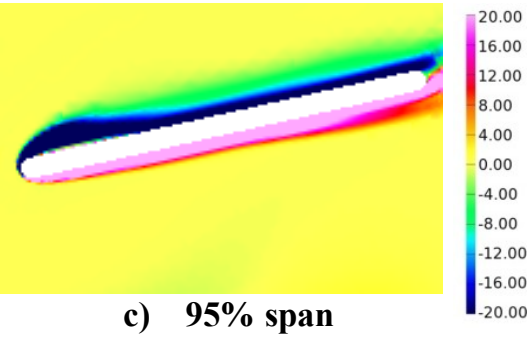

c) $95 \%$ span

Figure 47. Spanwise variation of the LEV of the hindwing at $180^{\circ}$ phase lag angle and $\mathbf{t} / \mathbf{T}=\mathbf{0 . 1 7 5}$.

For each case, the LEV is mostly unaffected by the tip vortices from midspan to about $75 \%$ span. Beyond $75 \%$, tip vortices begin to affect the LEV formation, and at $95 \%$ span, the LEV is partially suppressed by tip vortices. This affect is most noticeable for the single wing and the hindwing at $0^{\circ}$ and $90^{\circ}$ phase lag, where the maximum lift occurs near the middle of the downstroke and tip vortices have had time to develop. The hindwing at $180^{\circ}$ shows much less affect due to the tip vortices because the maximum lift occurs near the top of downstroke, when tip vortices are still small. Futhermore, the LEV that forms on the $180^{\circ}$ hindwing is much smaller compared to the other cases.

Tip vortices may play an important role in stabilizing the LEV for certain flapping motions that have translations above two chord lengths ${ }^{[57,58]}$. While the total stroke translation for the cases tested in this paper is only one chord length, it is still worthwhile to check if tip vortices play any role in stabilizing the LEV in the three dimensional cases. This was determined by comparing the vorticity contours of the three dimensional 
cases at midspan to the two dimensional vorticity contours. This is presented for the single wing and the $0^{\circ}, 90^{\circ}$, and $180^{\circ}$ hindwings in Figure 48-Figure 51, respectively.

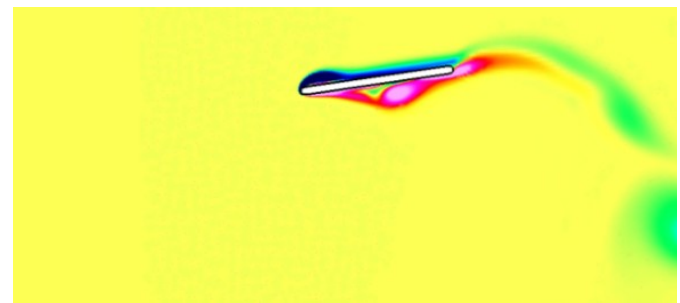

a) $2 \mathrm{D}, \mathrm{t} / \mathrm{T}=\mathbf{0 . 1 2}$ cycle time

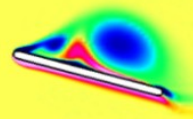

c) $2 \mathrm{D}, \mathrm{t} / \mathrm{T}=\mathbf{0 . 5 8}$ cycle time

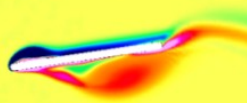

b) $3 \mathrm{D}, \mathrm{t} / \mathrm{T}=\mathbf{0 . 1 2}$ cycle time

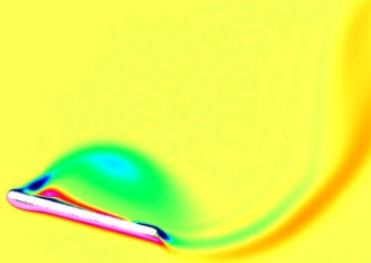

d) $3 \mathrm{D}, \mathrm{t} / \mathrm{T}=\mathbf{0 . 5 8}$ cycle time
20.00

16.00

12.00

8.00

4.00

$-0.00$

$-4.00$

$-8.00$

$-12.00$

$-16.00$

$-20.00$

(1)

Figure 48. Two dimensional vorticity contours of the single wing compared to the three dimensional vorticity contours at midspan. The LEV shed by the three dimensional wing is noticeably weaker than the two dimensional LEV.
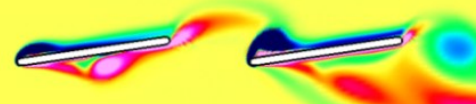
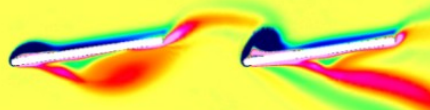

b) $3 \mathrm{D}, \mathrm{t} / \mathrm{T}=\mathbf{0 . 1 2}$ cycle time

a) $2 \mathrm{D}, \mathrm{t} / \mathrm{T}=\mathbf{0 . 1 2}$ cycle time

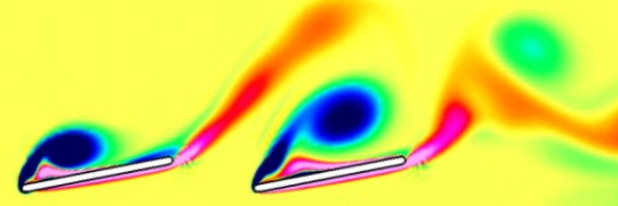

c) $2 \mathrm{D}, \mathrm{t} / \mathrm{T}=\mathbf{0 . 3 6}$ cycle time

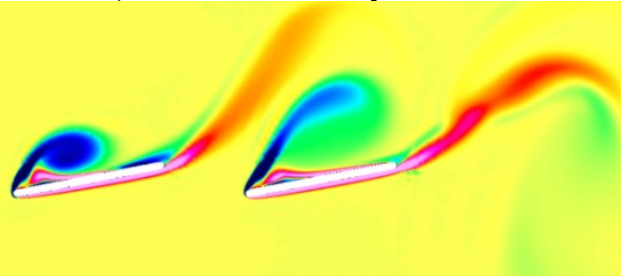

d) $3 \mathrm{D}, \mathrm{t} / \mathrm{T}=\mathbf{0 . 3 6}$ cycle time
20.00

16.00

12.00

8.00

4.00

$-0.00$

$-4.00$

$-8.00$

$-12.00$

$-16.00$

$-20.00$

Figure 49. Two dimensional vorticity contours of the hindwing at $0^{\circ}$ compared to the three dimensional vorticity contours at midspan. Similar to the single wing, the LEV shed by the three dimensional hindwing is noticeably weaker than the two dimensional LEV. 


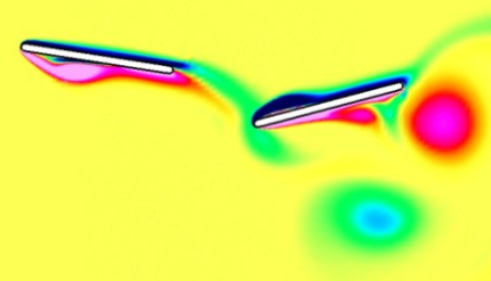

a) $2 \mathrm{D}, \mathrm{t} / \mathrm{T}=\mathbf{0 . 2 2}$ cycle time

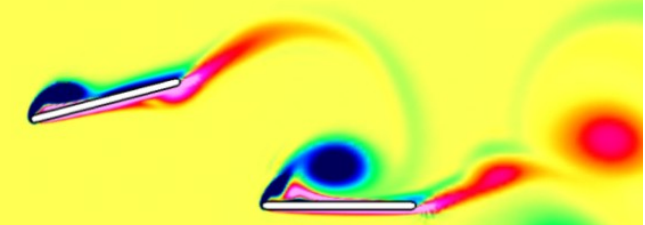

c) $2 \mathrm{D}, \mathrm{t} / \mathrm{T}=\mathbf{0 . 4 6}$ cycle time

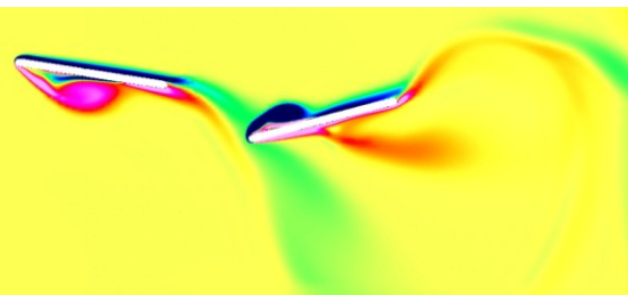

b) $3 \mathrm{D}, \mathrm{t} / \mathrm{T}=\mathbf{0 . 2 2}$ cycle time

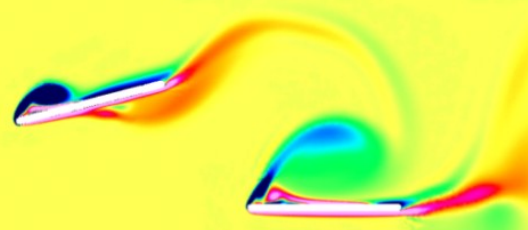

d) $3 \mathrm{D}, \mathrm{t} / \mathrm{T}=\mathbf{0 . 4 6}$ cycle time

Figure 50. Comparison between the two and three dimensional vorticity contours of the hindwing at $90^{\circ}$. In the three dimensional case, the LEV shed by the forewing is weaker, leading to a weaker interaction with the hindwing.

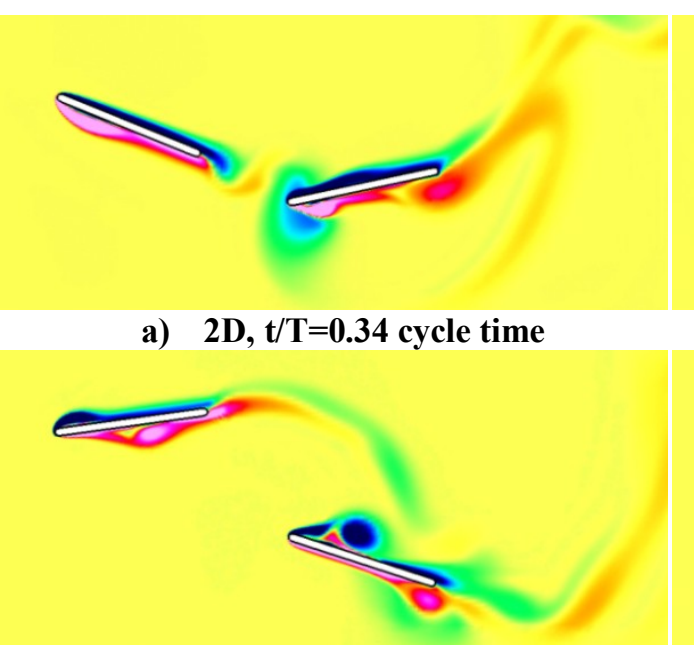

c) $2 \mathrm{D}, \mathrm{t} / \mathrm{T}=\mathbf{0 . 6 1}$ cycle time
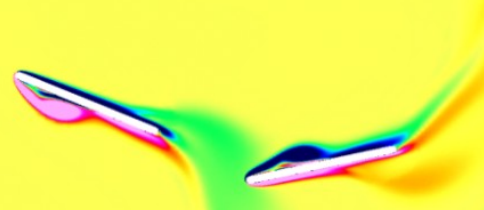

b) $3 D, t / T=0.34$ cycle time
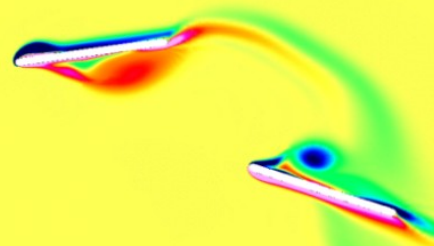

d) $3 \mathrm{D}, \mathrm{t} / \mathrm{T}=\mathbf{0 . 6 1}$ cycle time
$-20.00$

16.00

12.00

8.00

4.00

$-0.00$

$-4.00$

$-8.00$

$-12.00$

$-16.00$

$-20.00$

Figure 51. Comparison between the two and three dimensional vorticity contours of the hindwing at $180^{\circ}$. Due to the weaker LEV shed by the forewing, the interaction with the hindwing is weaker which results in the weaker LEV on the top of the three dimensional hindwing during the upstroke.

The results in Figure 48-Figure 51 suggest that there is no stabilization effect from the tip vortices as the LEV shedding pattern is the same in both the two and three dimensional results. The LEV's shed in the three dimensional cases, however; are noticeably weaker than their two dimensional counterparts. This is most likely due to the 
induced downwash of the tip vorticies reducing the effective angle of attack, and/or spanwise flow reducing the energy of the LEV (Figure 52). The reduction in LEV strength has an interesting effect on the hindwing at $180^{\circ}$ phase lag. At this phase angle, there is destructive interference between the vorticity shed from the forewing and the LEV generated by the hindwing. At the start of the upstroke, the hindwing passes through the LEV shed from the trailing edge of the forewing. Because the LEV shed from the forewing is weaker in the three dimensional case, the interference effect is weaker and the LEV generated on the bottom of the hindwing during the upstroke is stronger than the two dimensional case. This results in the large increase in thrust and negative lift exhibited by the three dimensional results in Figure 37. Similarly, the LEV generated on the top of the hindwing during the downstroke is also stronger than the 2D case, leading to an increase in positive lift and thrust generation during the downstroke as shown in Figure 37.

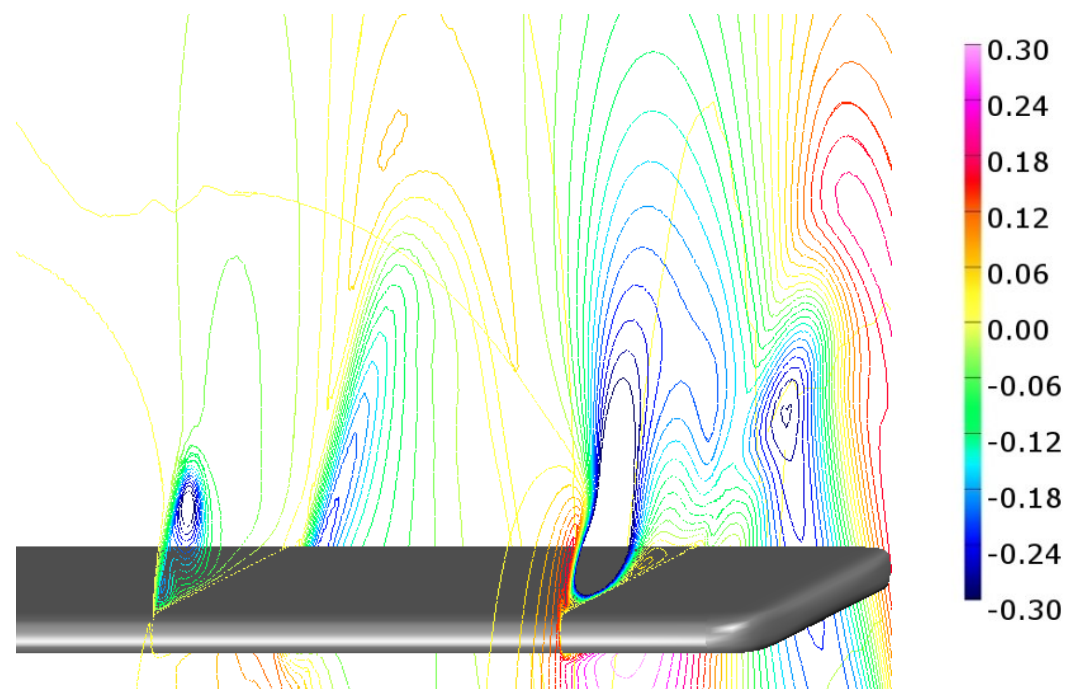

Figure 52. Spanwise velocity contours at $62.5 \%$ and $87.5 \%$ of span. The spanwise velocity is much stronger near the wingtips than near midspan. 


\section{3D SIMULATION AT RE=200}

While the $3 \mathrm{D}$ results at $\mathrm{Re}=5000$ provide useful insights, the high computational requirements necessitated the use of a coarse mesh in order keep the required computational time within a reasonable timeframe. Because of this, the $3 \mathrm{D}$ analysis from before was repeated at a Reynolds number of 200. The lower Reynolds number reduced the computational workload allowing for the use of a finer mesh. The 3D wing in this case had an aspect ratio of two and the spacing between the fore and hind wing was equal to one chord length.

\section{A. 2D Aerodynamic Force Results}

Figure 53 shows the transient lift and thrust coefficients of the hindwing in the $2 \mathrm{D}$ tandem configuration at the three tested phase angles $\left(0^{\circ}, 90^{\circ}, 180^{\circ}\right)$. The coefficients of the single $2 \mathrm{D}$ wing with the same kinematics are also shown for comparison purposes. At $\mathrm{t} / \mathrm{T}=0$, all the wings are at the beginning of the downstroke, and at $\mathrm{t} / \mathrm{T}=0.5$ they are at the start of the upstroke. 


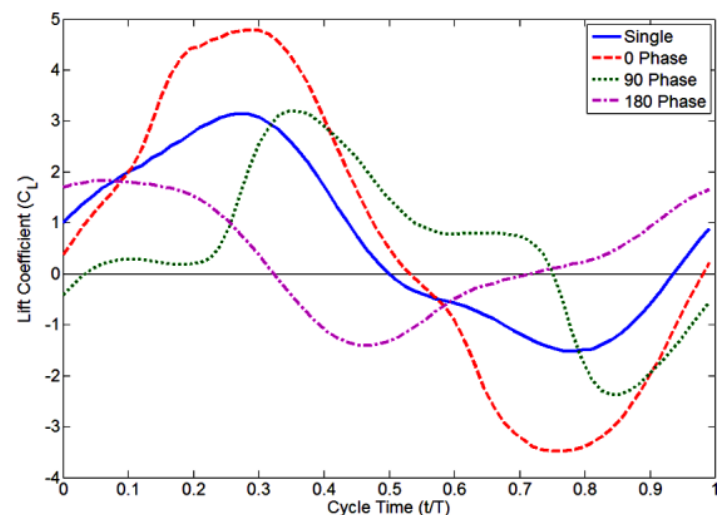

a) Lift

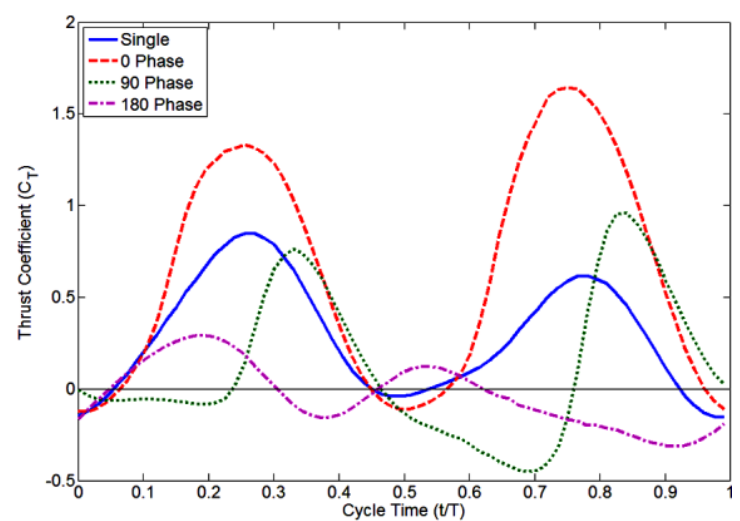

b) Thrust

Figure 53. The two dimensional transient lift and thrust coefficients of the hind wing at the three tested phase angles $\left(0^{\circ}, 90^{\circ}, 180^{\circ}\right)$ and the single wing over one flapping cycle.

It is clear the force coefficients of the hind wing are different from the ones of the single wing, indicating that the forewing has a significant effect on the force production of the hindwing. Furthermore, there are clear differences in the force coefficients among different phase angles, which show that variations in the phase angle between the fore and hind wings significantly alter the wing-wing interaction effect. When flapping in phase $\left(0^{\circ}\right.$ phase $)$, the force histories of the hind wing follow the same trend as the single wing but with much higher amplitude. Shifting the phase angle to $90^{\circ}$ causes a phase shift in the lift and thrust generation as well as a reduction in the lift and thrust amplitude from the $0^{\circ}$ phase case. The phase shift in lift is such that the hindwing produces positive lift through the first half of the upstroke and nearly zero lift through the first half of the downstroke. Another $90^{\circ}$ shift in the phase angle, to a total of $180^{\circ}$, causes a further phase shift in lift and thrust generation and an even greater reduction in the maximum lift and thrust generated on the upstroke and downstroke to below the peak values of the single wing. For this case, the hindwing produces positive lift through the second half of the upstroke and negative lift through the second half of the downstroke. 
Table 11 shows the cycle averaged lift, thrust, resultant and power coefficients for the hindwing at the three tested phase angles $\left(0^{\circ}, 90^{\circ}, 180^{\circ}\right)$ and the single wing. The lift, thrust, and resultant efficiencies are also shown in the table. The most beneficial value in each column is in bold. The power coefficient, $\mathrm{C}_{\mathrm{p}}$, which is a nondimensional measurement of the power required to actuate the wing during a single flapping cycle, was calculated using Equation 18. The lift, propulsive and resultant efficiencies were calculated using Equations 19-21.

\begin{tabular}{|c|c|c|c|c|c|c|c|}
\cline { 2 - 8 } \multicolumn{1}{c|}{} & $\overline{\mathbf{C}_{\mathbf{L}}}$ & $\overline{\mathbf{C}_{\mathbf{L}}}$ Eff. & $\overline{\mathbf{C}_{\mathbf{T}}}$ & $\overline{\mathbf{C}_{\mathbf{T}}}$ Eff. & $\overline{\mathbf{C}_{\mathbf{R}}}$ & $\overline{\mathbf{C}_{\mathbf{R}}}$ Eff. & Power \\
\hline Single & $\mathbf{0 . 6 7 8}$ & $62.2 \%$ & 0.291 & $26.7 \%$ & 0.738 & $67.7 \%$ & 1.090 \\
\hline 0 Hind & 0.562 & $29.5 \%$ & $\mathbf{0 . 6 4 7}$ & $\mathbf{3 4 . 0 \%}$ & $\mathbf{0 . 8 5 7}$ & $45.0 \%$ & 1.905 \\
\hline 90 Hind & 0.473 & $70.0 \%$ & 0.125 & $18.5 \%$ & 0.489 & $72.4 \%$ & 0.676 \\
\hline 180 Hind & 0.374 & $\mathbf{8 5 . 0 \%}$ & -0.034 & $-7.7 \%$ & 0.376 & $\mathbf{8 5 . 4 \%}$ & $\mathbf{0 . 4 4 0}$ \\
\hline
\end{tabular}

Table 11. Two dimensional cycle averaged values for the hindwings compared to a single wing.

Table 11 shows that, except for the lift coefficient, the tandem hind wing performs better than a single wing for all other values, depending on the phase angle. For a phase angle of $0^{\circ}$, the hind wing has the highest thrust and resultant coefficients as well as the highest propulsive efficiency. Switching to a phase angle of $180^{\circ}$, the hindwing has the highest lift and resultant efficiencies as well as the lowest power consumption. At a phase angle of $90^{\circ}$, the hindwing shows performance between that of the $0^{\circ}$ and $180^{\circ}$ cases.

Table 12 shows the results for the tandem configuration by averaging the lift, thrust, resultant and power coefficient between the fore and hind wing. Again, the most desirable result in each column is bolded. 


\begin{tabular}{|l|c|c|c|c|c|c|c|}
\cline { 2 - 8 } \multicolumn{1}{c|}{} & $\overline{\mathbf{C}_{\mathbf{L}}}$ & $\overline{\mathbf{C}_{\mathbf{L}}}$ Eff. & $\overline{\mathbf{C}_{\mathbf{T}}}$ & $\overline{\mathbf{C}_{\mathbf{T}}}$ Eff. & $\overline{\mathbf{C}_{\mathbf{R}}}$ & $\overline{\mathbf{C}_{\mathbf{R}}}$ Eff. & Power \\
\hline Single & 0.678 & $62.2 \%$ & 0.291 & $26.7 \%$ & 0.738 & $67.7 \%$ & 1.090 \\
\hline 0 Phase & $\mathbf{0 . 7 1 4}$ & $45.4 \%$ & $\mathbf{0 . 4 8 8}$ & $\mathbf{3 1 . 0 \%}$ & $\mathbf{0 . 8 6 5}$ & $54.9 \%$ & 1.574 \\
\hline 90 Phase & 0.649 & $68.6 \%$ & 0.225 & $23.7 \%$ & 0.686 & $72.5 \%$ & 0.946 \\
\hline 180 Phase & 0.574 & $\mathbf{7 5 . 0 \%}$ & 0.124 & $16.1 \%$ & 0.587 & $\mathbf{7 6 . 7 \%}$ & $\mathbf{0 . 7 6 5}$ \\
\hline
\end{tabular}

Table 12. Two dimensional cycle averaged values averaged between the fore and hind wing for each phase angle compared to a single wing.

The results in Table 12 show that the tandem wing can outperform the single wing in each category by altering the phase angle between the fore and hind wings. With a phase angle of $0^{\circ}$, the tandem configuration produces the largest cycle averaged forces and has the highest propulsive efficiency. At a phase angle of $180^{\circ}$ the tandem configuration has the highest lift and resultant efficiencies and the lowest power consumption. The $90^{\circ}$ case falls between the performance of the $0^{\circ}$ and $180^{\circ}$ cases. The ability to change the phasing between the fore and hind wings allows the tandem configuration to change its flight mode to obtain the desired performance, from high powered, high force generation at $0^{\circ}$, to low powered, high efficiency at $180^{\circ}$, to a compromise between the two at $90^{\circ}$.

\section{B. 2D Vorticity Contours}

The relationship between the lift and thrust production of the hind wing and the phase angle shows a strong correlation with the effect of the forewing on the hind wing LEVs. Figure 54-Figure 56 show the snapshots of vorticity contours at different time instants for the $0^{\circ}, 90^{\circ}$ and $180^{\circ}$ cases, respectively. The vorticity contours of the single wing are shown alongside each case for the sake of comparison.

The first row in Figure 54 show the single wing and $0^{\circ}$ phase case early in the downstroke. At this point, the hindwing passes through the vortex shed from the 
forewing, which interacts with the LEV that is forming on the top of the hindwing. The vortex from the forewing and the LEV from the hindwing both rotate clockwise $(\mathrm{CW})$. This interaction reinforces the LEV generated by the hindwing, increasing its size, as shown in Figure 54d, which shows the hindwing at $\mathrm{t} / \mathrm{T}=0.3$ when lift and thrust reach their peak. This interaction is repeated on the upstroke and the resulting LEV is shown shedding from the bottom of the hindwing in Figure 54b. The larger LEV results in the increase in lift and thrust generated on the downstroke and upstroke, as evidenced in Figure 53.

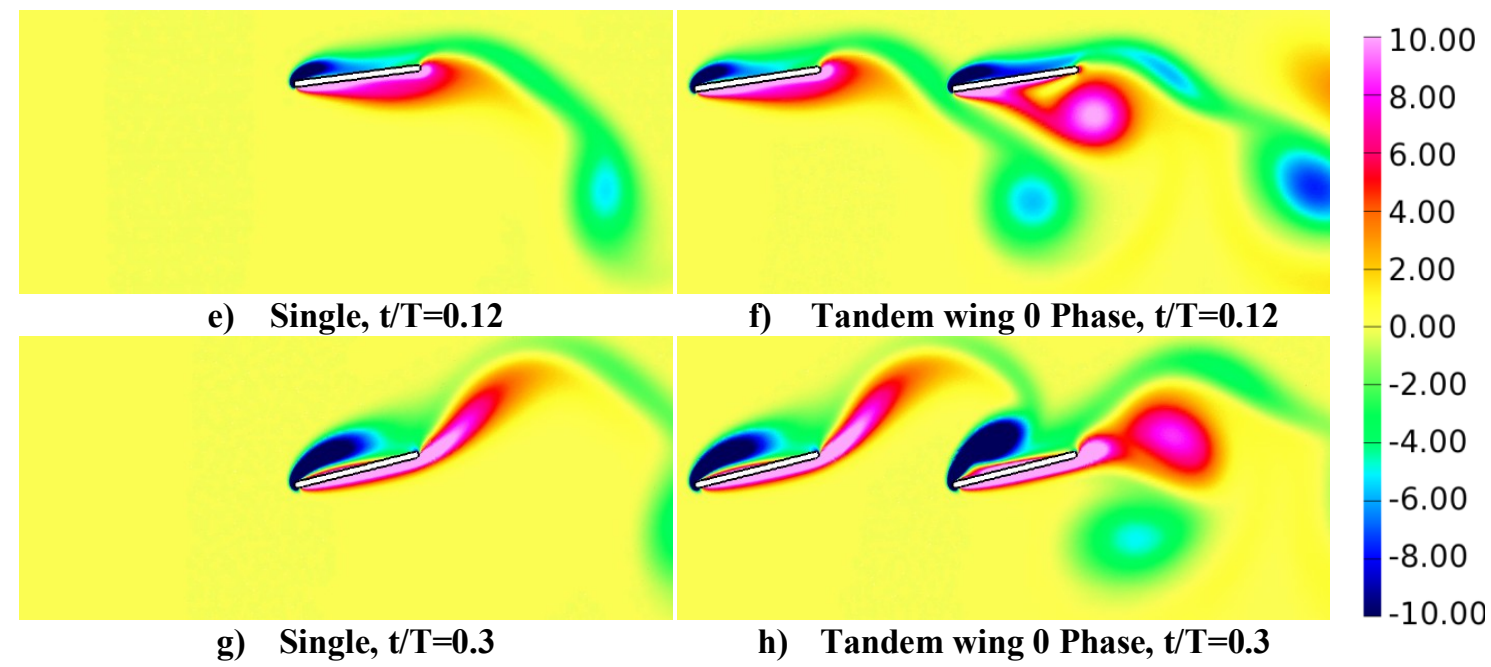

Figure 54. The vorticity contours illustrating the vortex interactions between the fore and hind wing with a phase angle of $0^{\circ}$. At this phase angle the vortex interactions are constructive and increase the size of the LEV generated by the hindwing. 


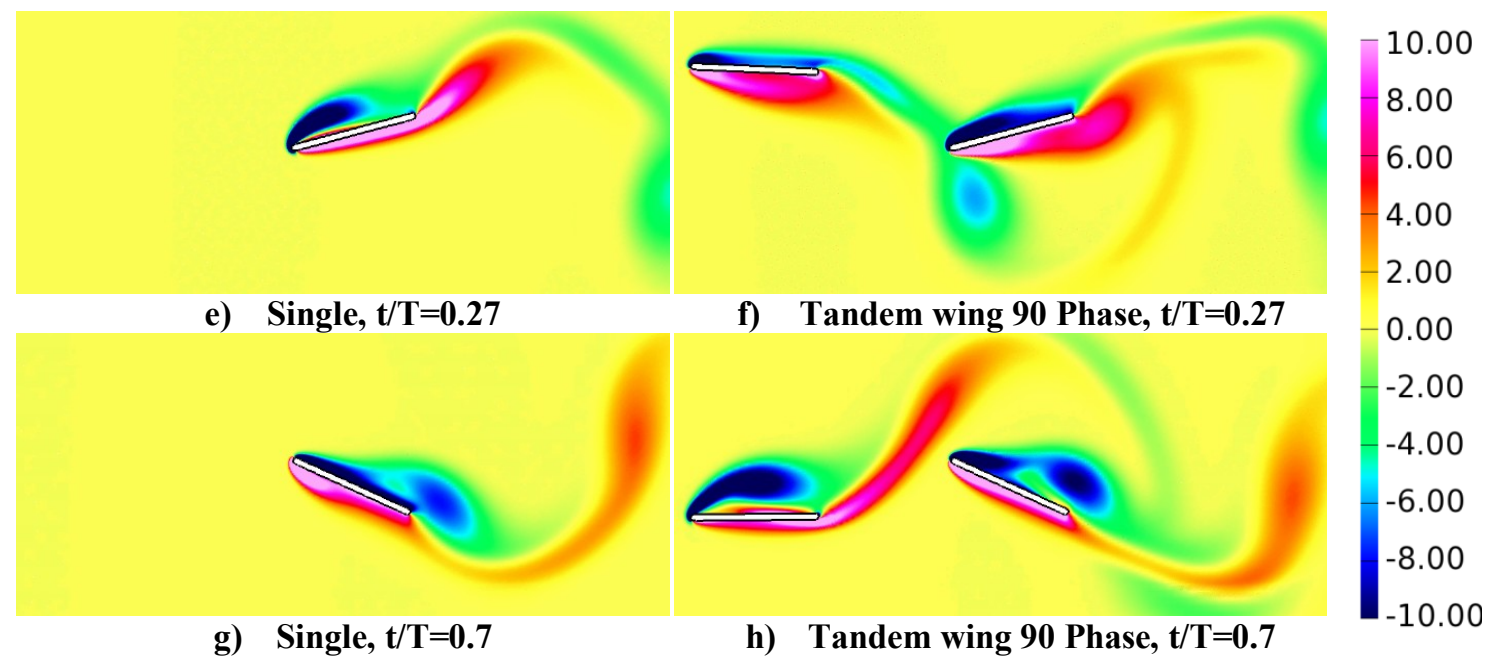

Figure 55. The vorticity contours illustrating the vortex interactions between the fore and hind wing with a phase angle of $90^{\circ}$. At this phase angle the vortex interactions cause an LEV to remain attached to the top of the hindwing through the first half of the upstroke.

The vortex interaction of the $90^{\circ}$ phase case (Figure 55) is similar to the $0^{\circ}$ phase case in some aspects. During the downstroke, the hindwing of the $90^{\circ}$ case also passes through the $\mathrm{CW}$ vortex shed from the forewing which interacts with $\mathrm{CW}$ LEV generated on the top of the hindwing; however, this interaction occurs later in the downstroke $(\mathrm{t} / \mathrm{T}=0.27)$ than the $0^{\circ}$ case. This causes a phase shift in the LEV generation of the hindwing. The interaction in the second half of the downstroke reinforces the CW LEV formation on the top of the hindwing, delays it shedding, and allows it to persist through half of the upstroke, as shown in Figure 55d. The interaction is then repeated during the second half of the upstroke, which reinforces the counterclockwise (CCW) LEV forming on the bottom of the hindwing which delays its shedding and allows it to remain attached through the first half of the downstroke, as shown in Figure 55b. By keeping the LEV attached to the top of the airfoil through the first half of the upstroke, the hindwing generates positive lift during that portion of the cycle. 


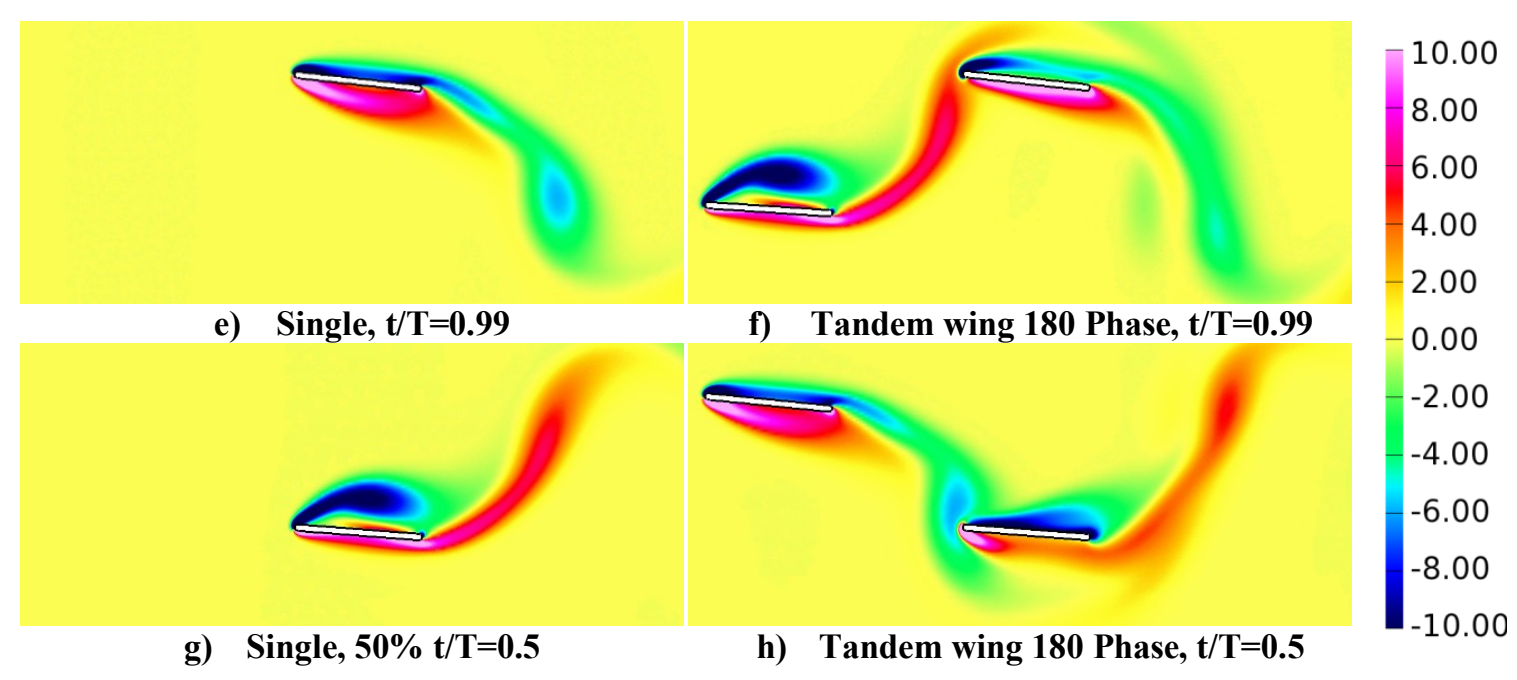

Figure 56. The vorticity contours illustrating the vortex interactions between the fore and hind wing with a phase angle of $180^{\circ}$. At this phase angle the vortex interactions are destructive and dampen the size of the LEV generated by the hindwing.

The vortex interactions that occur for the $180^{\circ}$ phase case (Figure 56) are nearly the opposite of $0^{\circ}$ phase case. At the start of the downstroke, the hindwing of the $180^{\circ}$ case passes through the $\mathrm{CCW}$ vortex shed from the forewing. This dampens out the $\mathrm{CW}$ LEV that would normally form on the top of the hindwing during the downstroke and instead a small CCW LEV is generated on the bottom, which can be seen in Figure 56d. This interaction is then repeated during the upstroke, which dampens out the CCW LEV that would normally form on the bottom of the hindwing. The hindwing instead generates a small CW LEV on the top, which is shown in Figure 56b. The resulting weak LEV formation leads to the weak lift and thrust production observed in Figure 53.

In general, the vortex interactions between the fore and hind wings can be characterized as either constructive or destructive. When the hindwing flaps in phase $\left(0^{\circ}\right.$ phase lag), constructive vortex interactions take place which reinforce the LEV generation of the hindwing, leading to large LEVs and the resulting increase in the peak lift and thrust. Conversely, the $180^{\circ}$ case exhibits destructive vortex interaction which 
dampens out LEV generation. This leads to weak LEV generation resulting in the weak lift and thrust production. The $90^{\circ}$ case falls in between the $0^{\circ}$ and $180^{\circ}$ cases, where the vortex interactions cause a phase shift in LEV production, resulting in a phase shift in the lift and thrust production. Furthermore, the vortex interactions of the $90^{\circ}$ and $180^{\circ}$ cases, result in the hindwing producing a significant amount of positive lift during the upstroke and negative lift during the downstroke. This results in the significant power reduction for both cases due to the direction of force production being coincident to the direction of wing motion for a significant portion of the flapping cycle.

\section{3D Aerodynamic Force Results}

Figure 57 shows the transient lift and thrust coefficients of the hind wing in the 3D tandem configuration at the three tested phase angles $\left(0^{\circ}, 90^{\circ}, 180^{\circ}\right)$ and a single wing over one flapping cycle. At $\mathrm{t} / \mathrm{T}=0$, all the wings are at the beginning of the downstroke, and at $\mathrm{t} / \mathrm{T}=0.5$ they are at the start of the upstroke.

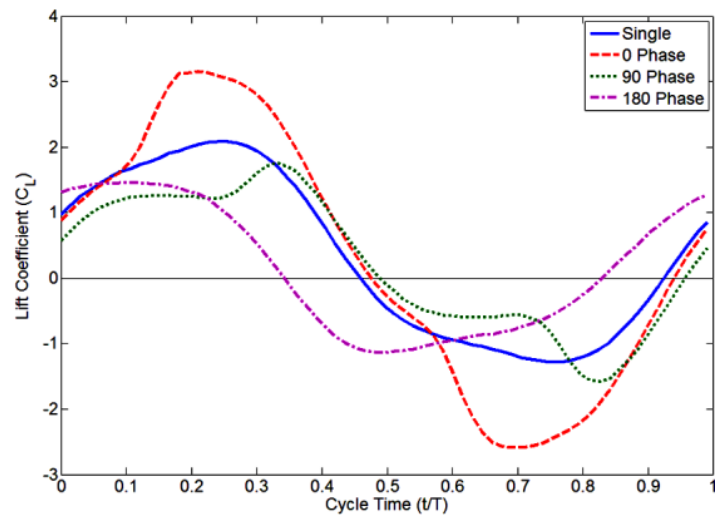

c) Lift

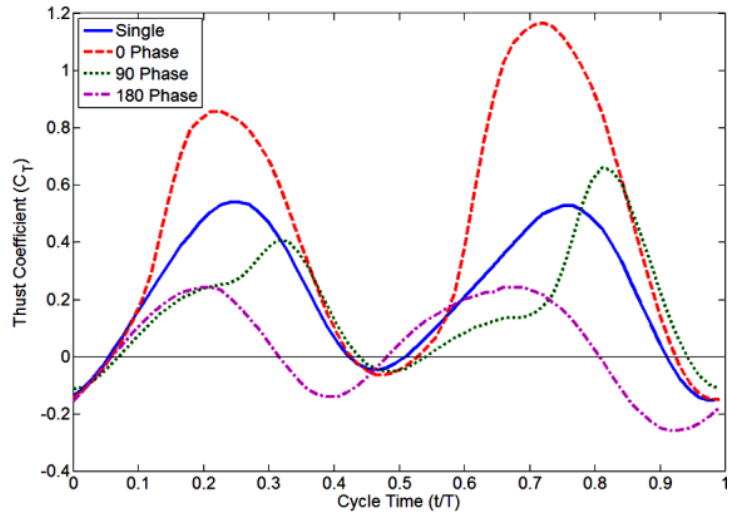

d) Thrust

Figure 57. The transient lift and thrust coefficients of the 3D hind wing at the three tested phase angles $\left(0^{\circ}, 90^{\circ}, 180^{\circ}\right)$ and the single wing over one flapping cycle.

The relationship between the phase angle and the lift and thrust production of the hindwing is very similar to that of the $2 \mathrm{D}$ case shown in Figure 53. For the $0^{\circ}$ phase lag case the hindwing shows significantly higher peak lift and thrust during the upstroke and 
downstroke. For the $90^{\circ}$ phase lag case the hindwing exhibits a slight phase lag in the lift and thrust with similar magnitudes of peak lift and thrust to the single wing. The $180^{\circ}$ hindwing shows a phase lag in the lift and thrust with a significantly lower magnitude of peak lift and thrust than the single wing. The differences in the two dimensional and three dimensional transient force results are displayed more clearly in Figure 58-Figure 61 which plot the 2D and 3D lift and thrust together for the single wing and the tandem hindwing at the three tested phase angles. The main difference between the 2D and 3D force results is that all of the 3D cases show a reduction in the lift and thrust peak magnitude compared to the two dimensional results. This reduction may be due to the tip vortices in the $3 \mathrm{D}$ cases. The one exception is the hindwing at $180^{\circ}$ phase lag during the upstroke where the three dimensional results show a larger increase in the thrust over the two dimensional results.

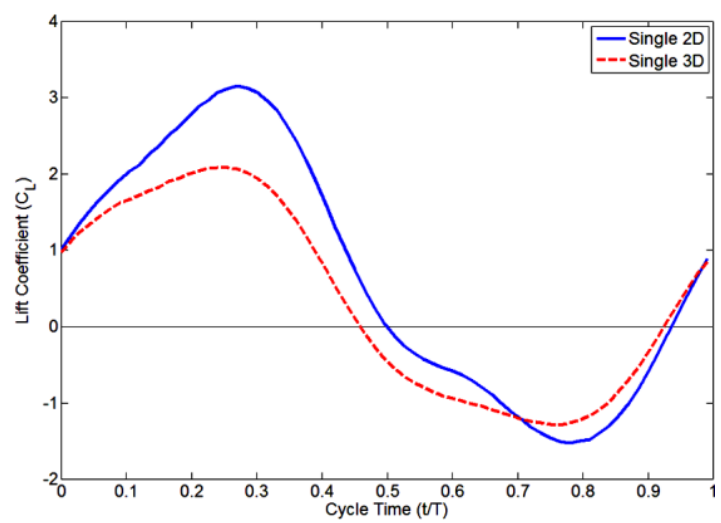

c) Lift

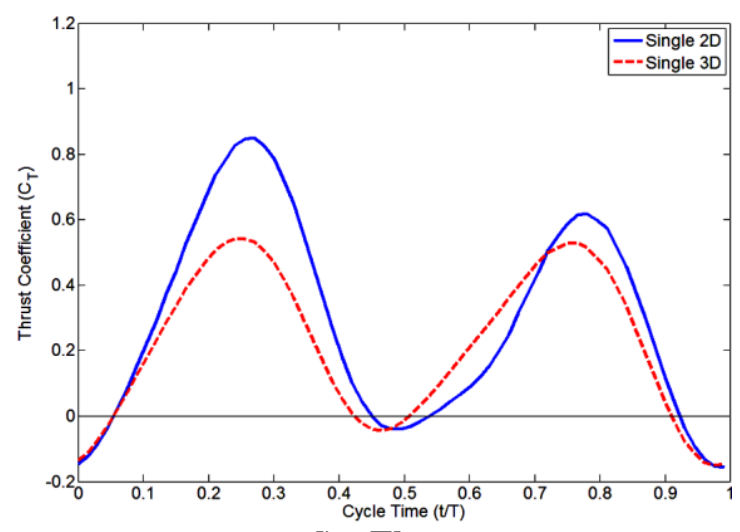

d) Thrust

Figure 58. The transient lift and thrust coefficients of the 3D single wing compared to the same results for the $2 D$ case. 


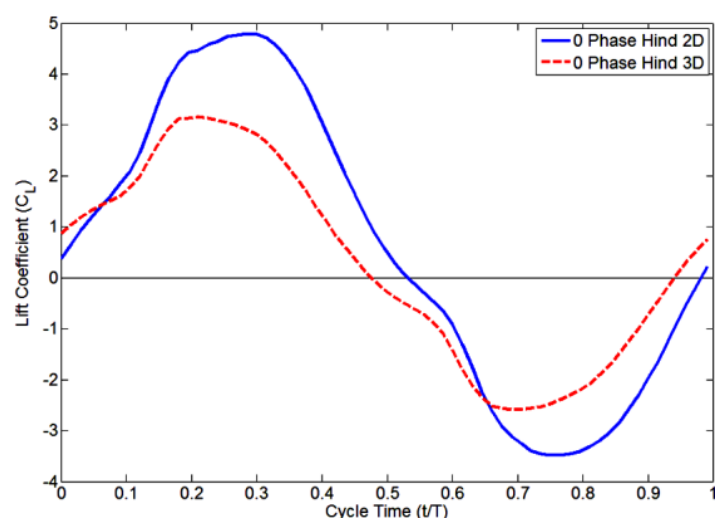

c) Lift

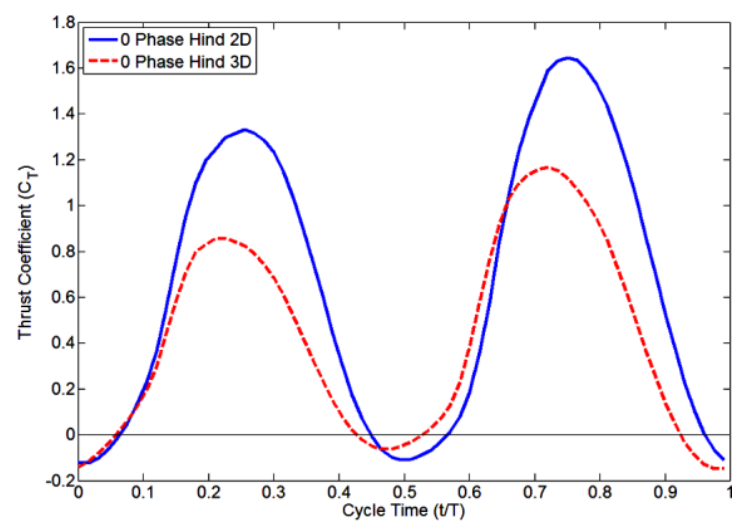

d) Thrust

Figure 59. The transient lift and thrust coefficients of the $3 D$ hindwing at $0^{\circ}$ compared to the same results for the $2 \mathrm{D}$ case.

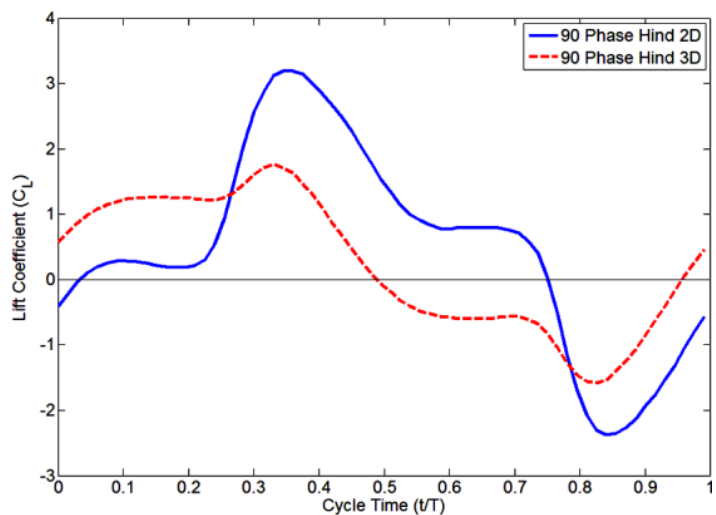

c) Lift

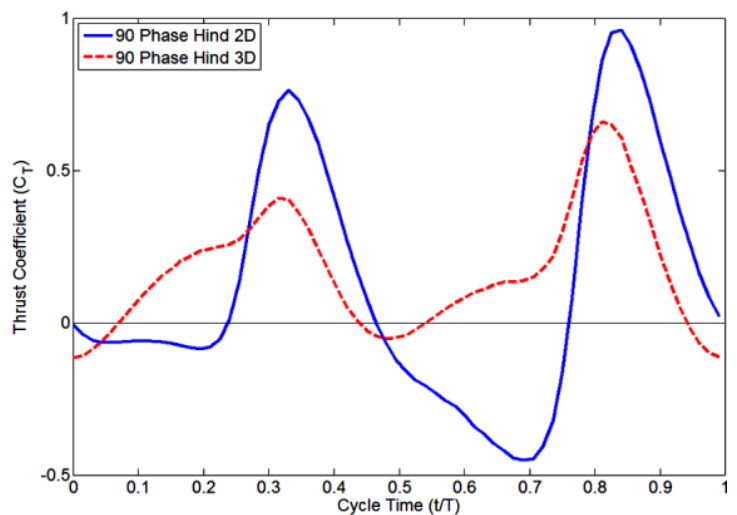

d) Thrust

Figure 60. The transient lift and thrust coefficients of the $3 D$ hindwing at $90^{\circ}$ compared to the same results for the $2 \mathrm{D}$ case.

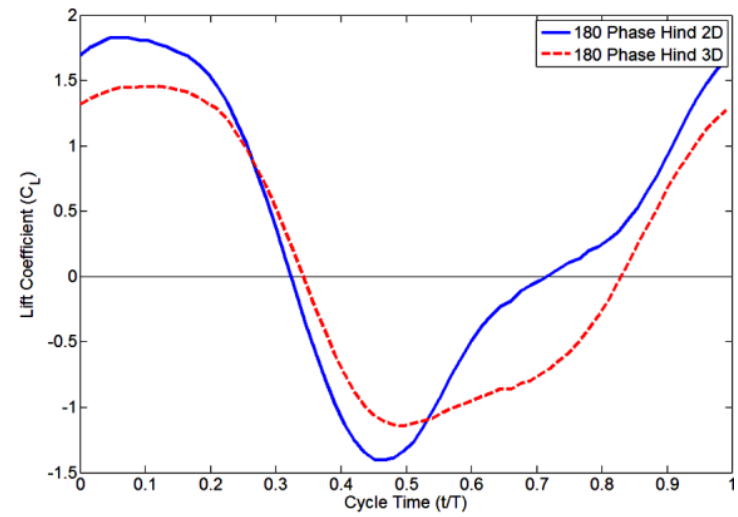

c) Lift

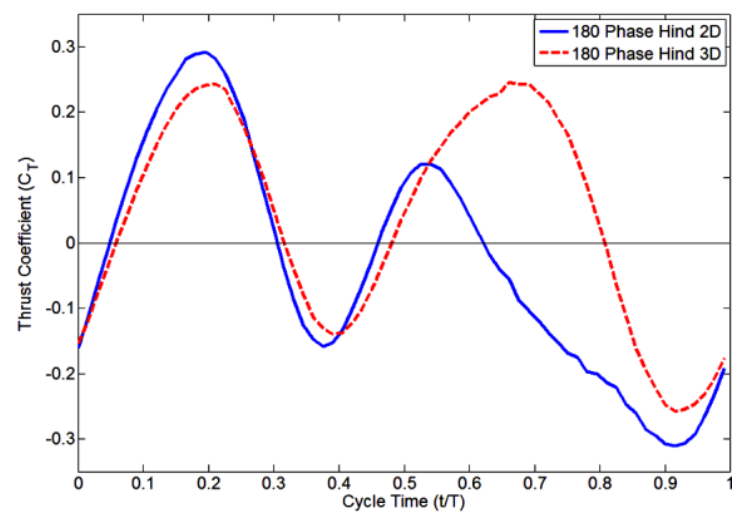

d) Thrust

Figure 61. The transient lift and thrust coefficients of the $3 D$ hindwing at $180^{\circ}$ compared to the same results for the $2 \mathrm{D}$ case.

Table 13 compares the cycle averaged parameters between the hindwing and a standalone single wing. The most beneficial value in each column is in bold. 


\begin{tabular}{|l|c|c|c|c|c|c|c|}
\cline { 2 - 8 } \multicolumn{1}{c|}{} & $\overline{\mathbf{C}_{\mathbf{L}}}$ & $\overline{\mathbf{C}_{\mathbf{L}}}$ Eff. & $\overline{\mathbf{C}_{\mathbf{T}}}$ & $\overline{\mathbf{C}_{\mathbf{T}}}$ Eff. & $\overline{\mathbf{C}_{\mathbf{R}}}$ & $\overline{\mathbf{C}_{\mathbf{R}}}$ Eff. & Power \\
\hline Single & $\mathbf{0 . 3 3 3}$ & $\mathbf{4 0 . 8 \%}$ & 0.221 & $27.1 \%$ & 0.400 & $\mathbf{4 8 . 9 \%}$ & 0.817 \\
\hline 0 Hind & 0.265 & $20.7 \%$ & $\mathbf{0 . 4 2 4}$ & $\mathbf{3 3 . 2 \%}$ & $\mathbf{0 . 5 0 0}$ & $39.1 \%$ & 1.278 \\
\hline 90 Hind & 0.224 & $33.2 \%$ & 0.173 & $25.7 \%$ & 0.283 & $42.0 \%$ & 0.674 \\
\hline 180 Hind & 0.154 & $34.0 \%$ & 0.035 & $7.7 \%$ & 0.158 & $34.9 \%$ & $\mathbf{0 . 4 5 3}$ \\
\hline
\end{tabular}

Table 13. Three dimensional cycle averaged values for the hindwings compared to a single wing.

The three dimensional results in Table 13 show a similar relationship between the phase angle and the cycle averaged results of the hindwing as the two dimensional results shown in Table 11. At $0^{\circ}$ phase lag the hindwing generates the highest thrust and resultant coefficients as well as the highest propulsive efficiency, while switching to $180^{\circ}$ requires the lowest power coefficient and also increases the lift efficiency. The three dimensional results; however, indicate that at $90^{\circ}$ phase lag, the hindwing has the highest resultant efficiency among the three tested phase angles, while the $180^{\circ}$ hindwing has the lowest resultant efficiency; whereas, the two dimensional results showed that the $180^{\circ}$ hindwing had the highest resultant efficiency. Furthermore, while the two and three dimensional results both show that the single wing has a higher lift coefficient than any of the hindwings, the three dimensional results also show that the single wing has the highest lift and resultant efficiencies; whereas, the two dimensional results indicated that the $180^{\circ}$ hindwing had the highest lift and resultant efficiencies.

Table 14 shows the results for the tandem configuration by averaging the values between the fore and hind wing. Again, the most desirable result in each column is bolded. 


\begin{tabular}{|l|c|c|c|c|c|c|c|}
\cline { 2 - 8 } \multicolumn{1}{c|}{} & $\overline{\mathbf{C}_{\mathbf{L}}}$ & $\overline{\mathbf{C}_{\mathbf{L}}}$ Eff. & $\overline{\mathbf{C}_{\mathbf{T}}}$ & $\overline{\mathbf{C}_{\mathbf{T}}}$ Eff. & $\overline{\mathbf{C}_{\mathbf{R}}}$ & $\overline{\mathbf{C}_{\mathbf{R}}}$ Eff. & Power \\
\hline Single & $\mathbf{0 . 3 3 3}$ & $\mathbf{4 0 . 8 \%}$ & 0.221 & $27.1 \%$ & 0.400 & $\mathbf{4 8 . 9 \%}$ & 0.817 \\
\hline 0 Phase & 0.313 & $28.9 \%$ & $\mathbf{0 . 3 3 3}$ & $\mathbf{3 0 . 7 \%}$ & $\mathbf{0 . 4 5 7}$ & $42.2 \%$ & 1.082 \\
\hline 90 Phase & 0.285 & $37.1 \%$ & 0.204 & $26.6 \%$ & 0.350 & $45.6 \%$ & 0.768 \\
\hline 180 Phase & 0.244 & $38.2 \%$ & 0.129 & $20.2 \%$ & 0.276 & $43.2 \%$ & $\mathbf{0 . 6 4 0}$ \\
\hline
\end{tabular}

Table 14. Three dimensional cycle averaged values averaged between the fore and hind wing for each phase angle compared to a single wing.

The same trends shown in Table 13 for the hindwings individually are mirrored in Table 14 for the tandem configuration as a whole. Like the results in Table 13, the three dimensional results in Table 14 show a similar relationship between the cycle averaged values and the phase angle. Unlike the two dimensional results; however, the three dimensional results do not show higher performance of the tandem wing than the single wing for all values. Instead, the three dimensional results show that the single wing has a higher lift coefficient and higher lift and resultant efficiencies than the tandem configuration. Also, in general, the three dimensional results in Table 13 and Table 14 exhibit lower force coefficients and efficiencies than the two dimensional results in Table 11 and Table 12.

\section{3D Vorticity Contours}

The iso-surface vorticity contours for the three dimensional cases are presented in Figure 62-Figure 64. To ensure a fair comparison with the $2 \mathrm{D}$ results, only the component in the spanwise direction is shown. The iso-surface contours of the $3 \mathrm{D}$ single wing are shown alongside the tandem wing for comparison. 


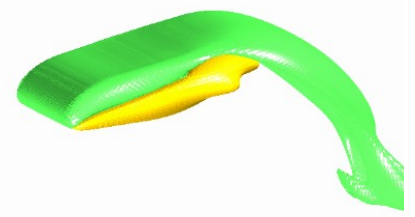

e) Single, $t / T=0.12$

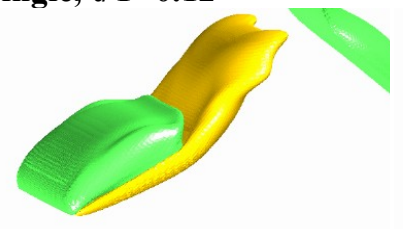

g) Single, $t / \mathbf{T}=\mathbf{0 . 3}$

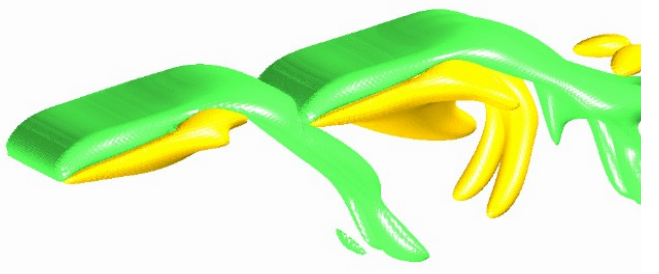

f) Tandem wing 0 Phase, $t / T=0.12$

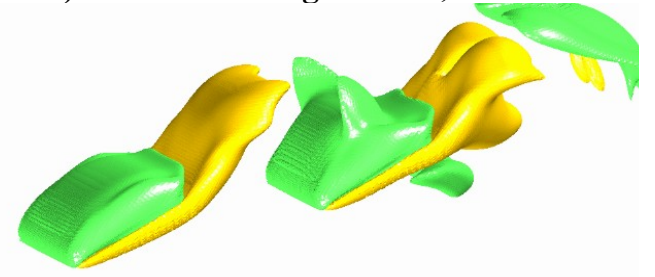

h) Tandem wing 0 Phase, $t / T=0.3$
10.00

8.00

6.00

4.00

2.00

0.00

$-2.00$

$-4.00$

$-6.00$

$-8.00$

$-10.00$

Figure 62. The iso-surface contours of the vorticity in the spanwise direction, illustrating the vortex interactions between the fore and hind wing with a phase angle of $0^{\circ}$. The three dimensional iso-surfaces show the same constructive interaction observed in the $2 \mathrm{D}$ case.

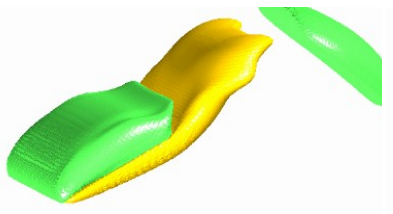

e) Single, $\mathbf{t} / \mathbf{T}=\mathbf{0 . 2 7}$

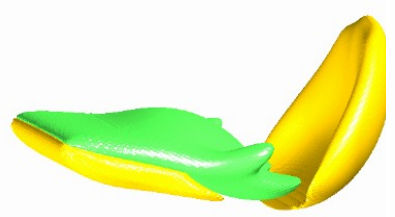

g) Single, $t / \mathbf{T}=\mathbf{0 . 7}$

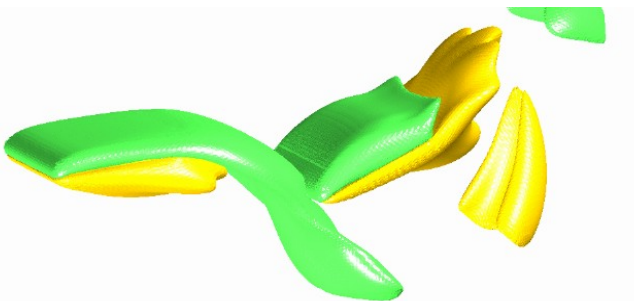

f) Tandem wing 90 Phase, $t / T=0.27$

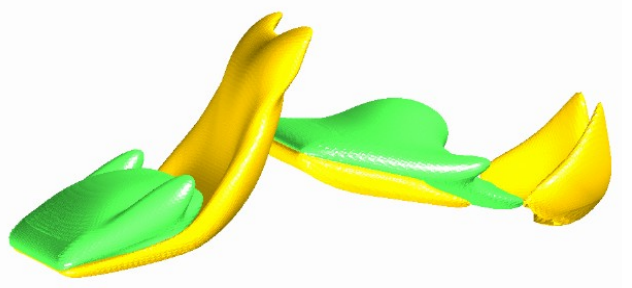

h) Tandem wing 90 Phase, t/T=0.7
$-10.00$

8.00

6.00

4.00

2.00

0.00

$-2.00$

$-4.00$

$-6.00$

$-8.00$

$-10.00$

Figure 63. The iso-surface contours of the vorticity in the spanwise direction, illustrating the vortex interactions between the fore and hind wing with a phase angle of $90^{\circ}$. 

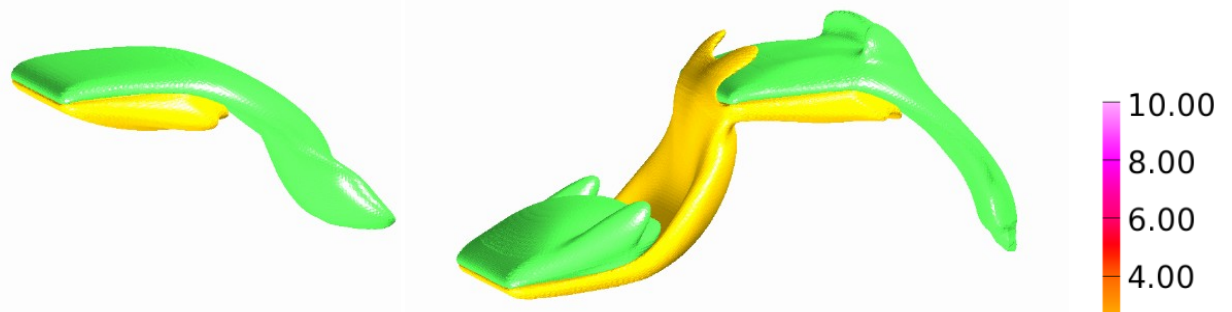

e) Single, $t / T=0.99$

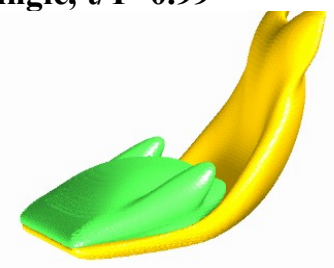

f) Tandem wing 180 Phase, $t / T=0.99$

2.00

0.00

$-2.00$

$-4.00$

$-6.00$

$-8.00$

g) Single, $t / T=0.5$

h) Tandem wing 180 Phase, $t / T=0.5$

Figure 64. The three dimensional iso-surface contours of the vorticity component in the spanwise direction, illustrating the vortex interactions between the fore and hind wing with a phase angle of $180^{\circ}$. The three dimensional iso-surfaces show the same destructive interaction observed in the $2 \mathrm{D}$ case.

The vortex interactions shown in the $3 \mathrm{D}$ cases are very similar to those in the $2 \mathrm{D}$ cases. In the $0^{\circ}$ phase lag case, the vortex shed from the forewing strengthens the same sign LEV on the hind wing; in the $180^{\circ}$ case, the vortex from the forewing dampens the opposite sign LEV on the hind wing.

\section{E. Effect of Tip Vortices on LEV}

To understand the interactions between the tip vortices and LEV, we show the LEV at three different positions along the span $(50 \%, 75 \%, 95 \%)$. Results from both single wing and hindwing are shown in Figure 65-Figure 68. The vorticity contours are shown during the downstroke for each case, at the time instant where the case displays peak force production $\left(\mathrm{t} / \mathrm{T}=0.3\right.$ for the single wing and the hindwing at $0^{\circ}$ phase lag angle, $\mathrm{t} / \mathrm{T}=0.35$ for the hindwing at $90^{\circ}$ phase lag angle and $\mathrm{t} / \mathrm{T}=0.1$ for the hindwing at $180^{\circ}$ phase lag angle). 


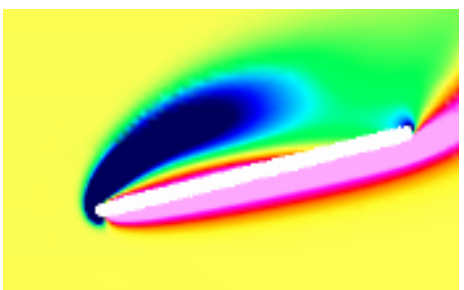

d) $50 \%$ span

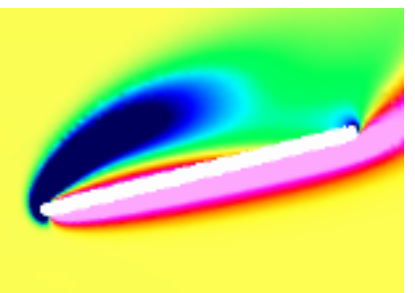

e) $75 \%$ span

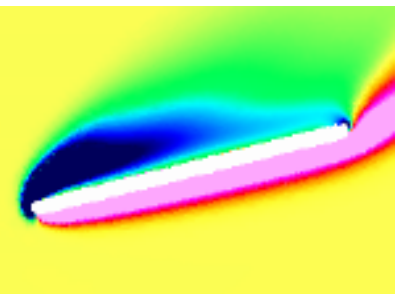

f) $95 \%$ span

Figure 65.Spanwise variation of the $\mathrm{LEV}$ of the single wing at $t / T=0.3$.

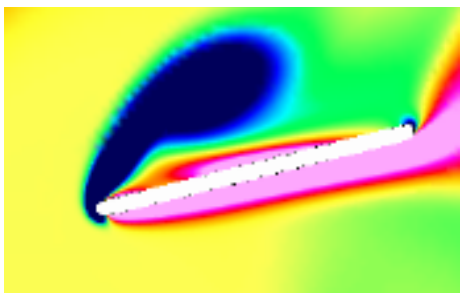

d) $50 \%$ span

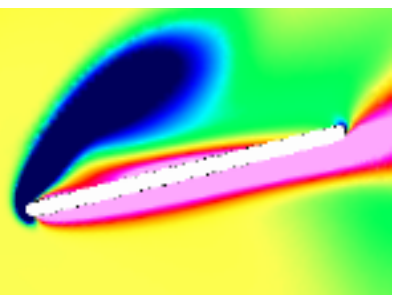

e) $75 \%$ span

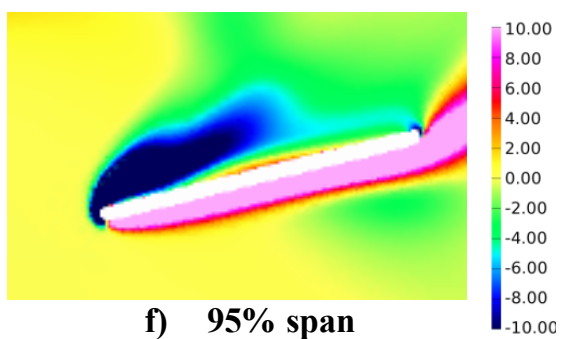

f) $95 \%$ span

Figure 66. Spanwise variation of the LEV of the hindwing at $0^{\circ}$ phase lag angle and $\mathbf{t} / \mathbf{T}=\mathbf{0 . 3}$.

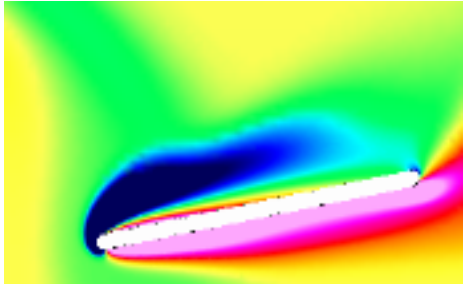

d) $\mathbf{5 0 \%}$ span

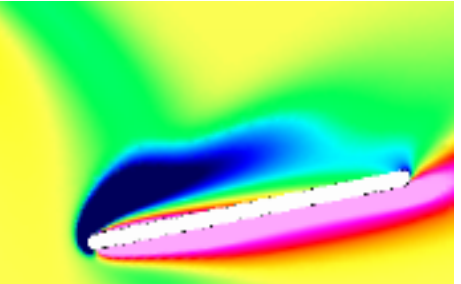

e) $75 \%$ span

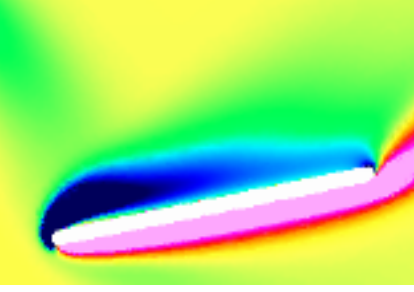

f) $95 \%$ span

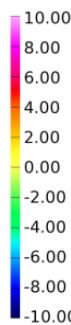

Figure 67. Spanwise variation of the LEV of the hindwing at $90^{\circ}$ phase lag angle and $\mathbf{t} / \mathbf{T}=\mathbf{0 . 3 5}$.

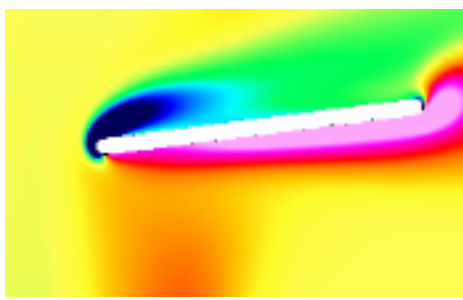

d) $50 \%$ span

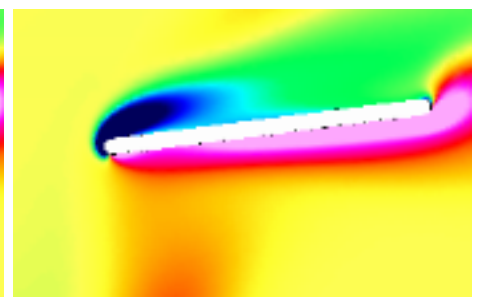

e) $75 \%$ span

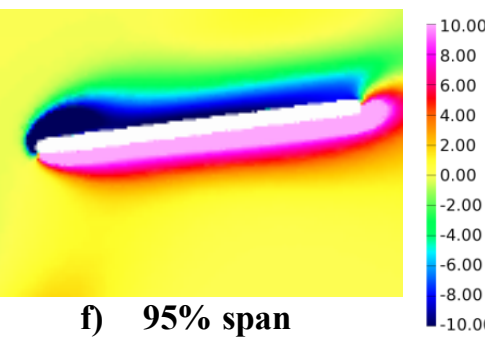

f) $95 \%$ span

Figure 68. Spanwise variation of the LEV of the hindwing at $180^{\circ}$ phase lag angle and $\mathbf{t} / \mathbf{T}=\mathbf{0 . 1}$.

For each case, the LEV is mostly unaffected by the tip vortices from midspan to about $75 \%$ span. Beyond $75 \%$, tip vortices begin to affect the LEV formation, and at $95 \%$ span, the LEV is partially suppressed by tip vortices. This affect is most noticeable for the single wing and the hindwing at $0^{\circ}$ and $90^{\circ}$ phase lag, where the maximum lift occurs 
near the middle of the downstroke and tip vortices have had time to develop. The hindwing at $180^{\circ}$ shows much less affect due to the tip vortices because the maximum lift occurs near the top of downstroke, when tip vortices are still small. This may partially explain why the force production of the three dimensional $180^{\circ}$ hindwing most closely approximates the two dimensional results during the downstroke (Figure 61).

Tip vortices may play an important role in stabilizing the LEV for certain flapping motions that have translations above two chord lengths. ${ }^{[57,58]}$ While the total stroke translation for the cases tested in this paper is only one chord length, it is still worthwhile to check if tip vortices play any role in stabilizing the LEV in the three dimensional cases. This was determined by comparing the vorticity contours of the three dimensional cases at midspan to the two dimensional vorticity contours. This is presented for the single wing and the $0^{\circ}, 90^{\circ}$, and $180^{\circ}$ hindwings in Figure 69-Figure 72, respectively.

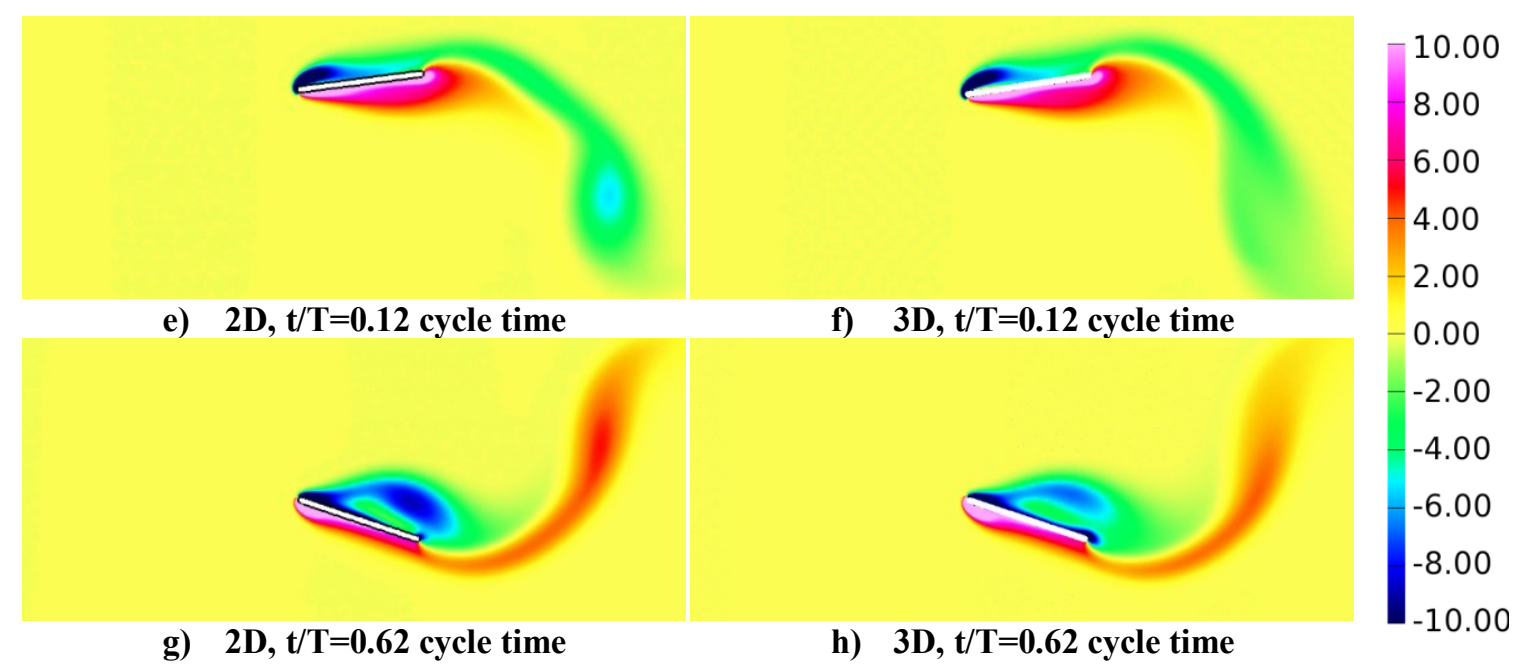

Figure 69. Two dimensional vorticity contours of the single wing compared to the three dimensional vorticity contours at midspan. The LEV shed by the three dimensional wing is noticeably weaker than the two dimensional LEV. 


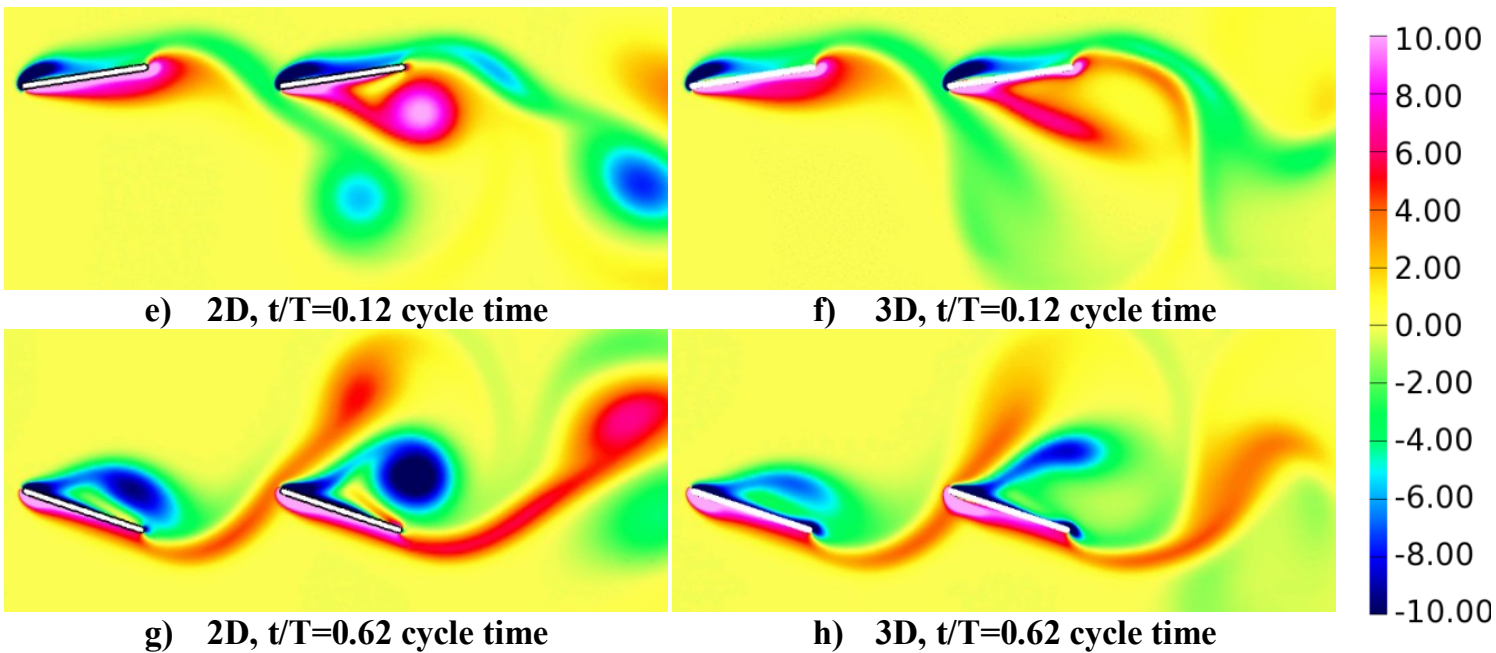

g) $2 \mathrm{D}, \mathrm{t} / \mathrm{T}=\mathbf{0 . 6 2}$ cycle time

h) $3 \mathrm{D}, \mathrm{t} / \mathrm{T}=\mathbf{0 . 6 2}$ cycle time

Figure 70. Two dimensional vorticity contours of the hindwing at $0^{\circ}$ compared to the three dimensional vorticity contours at midspan. Similar to the single wing, the LEV shed by the three dimensional hindwing is noticeably weaker than the two dimensional LEV.

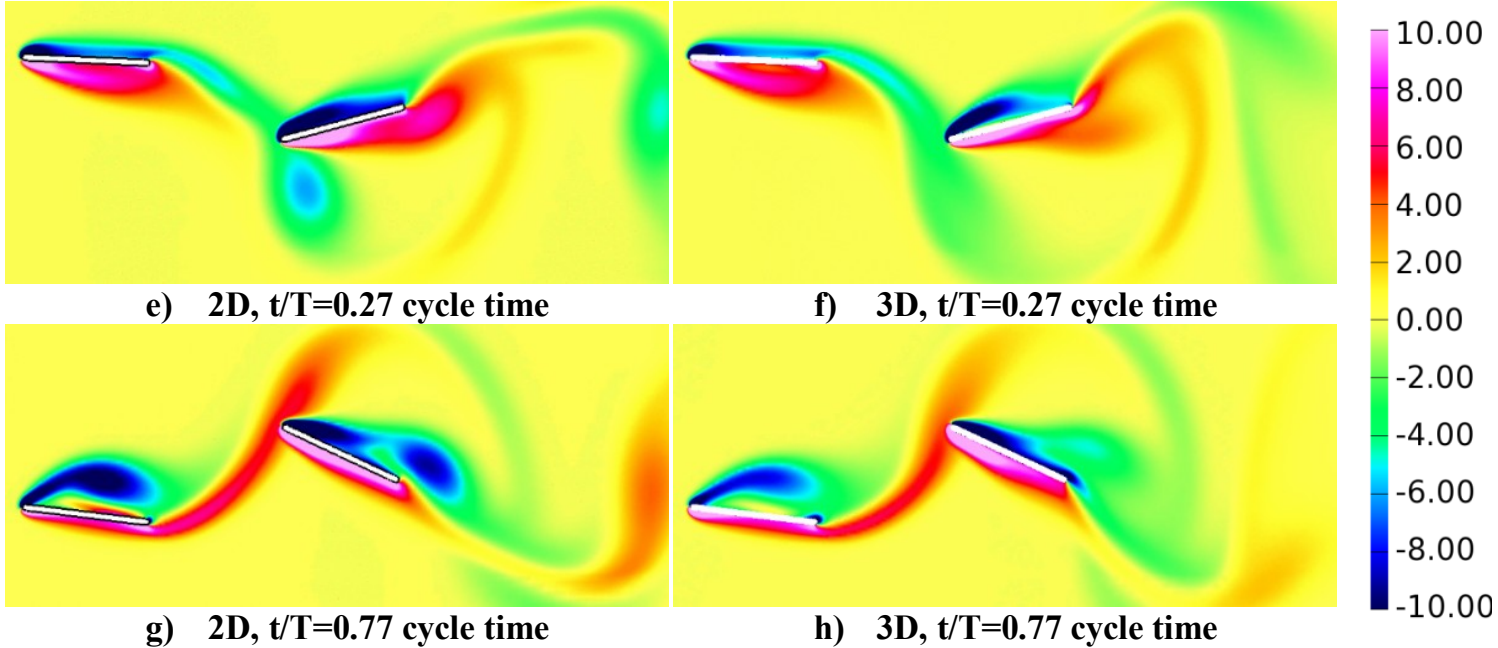

Figure 71. Comparison between the two and three dimensional vorticity contours of the hindwing at $90^{\circ}$. In the three dimensional case, the LEV shed by the forewing is weaker, leading to a weaker interaction with the hindwing which results in the stronger LEV on the bottom of the three dimensional hindwing during the upstroke. 


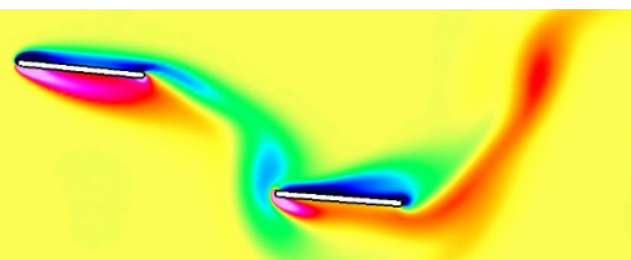

e) $2 \mathrm{D}, \mathrm{t} / \mathrm{T}=\mathbf{0 . 5 0}$ cycle time

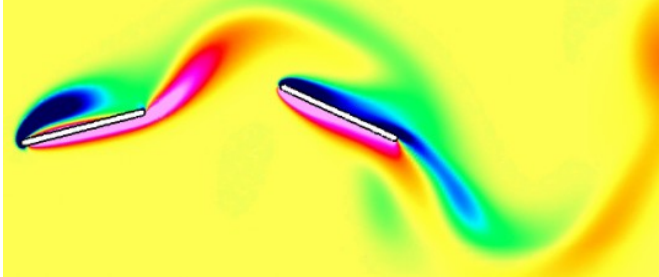

g) $2 \mathrm{D}, \mathrm{t} / \mathrm{T}=\mathbf{0 . 7 7}$ cycle time

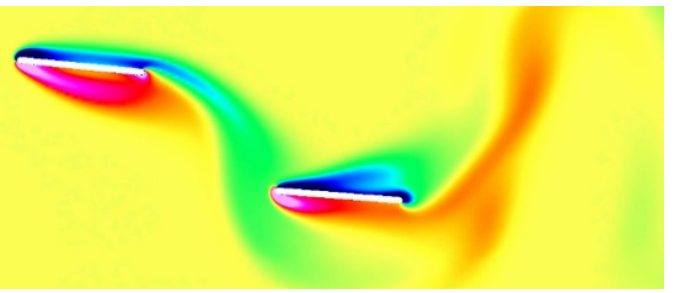

f) $3 D, t / T=0.50$ cycle time

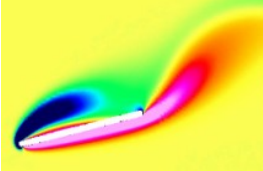

h) $3 \mathrm{D}, \mathrm{t} / \mathrm{T}=\mathbf{0 . 7 7}$ cycle time
10.00

8.00

6.00

4.00

2.00

0.00

$-2.00$

$-4.00$

$-6.00$

$-8.00$

$-10.00$

Figure 72. Comparison between the two and three dimensional vorticity contours of the hindwing at $180^{\circ}$. Due to the weaker LEV shed by the forewing, the interaction with the hindwing is weaker which results in the stronger LEV on the bottom of the three dimensional hindwing during the upstroke.

The results in Figure 69-Figure 72 suggest that there is no stabilization effect from the tip vortices as the LEV shedding pattern is the same in both the two and three dimensional results. The LEV's shed in the three dimensional cases, however; are noticeably weaker than their two dimensional counterparts. We hypothesis that this is due to the induced downwash of the tip vorticies reducing the effective angle of attack, and/or spanwise flow reducing the energy of the LEV (Figure 73). The reduction in LEV strength has an interesting effect on the hindwing at $180^{\circ}$ phase lag. At this phase angle, there is destructive interference between the vorticity shed from the forewing and the LEV generated by the hindwing. At the start of the upstroke, the hindwing passes through the LEV shed from the trailing edge of the forewing. Because the LEV shed from the forewing is weaker in the three dimensional case, the interference effect is weaker and the LEV generated on the bottom of the hindwing during the upstroke is stronger than the two dimensional case. This results in the large increase in thrust and negative lift exhibited by the three dimensional results in Figure 61. 


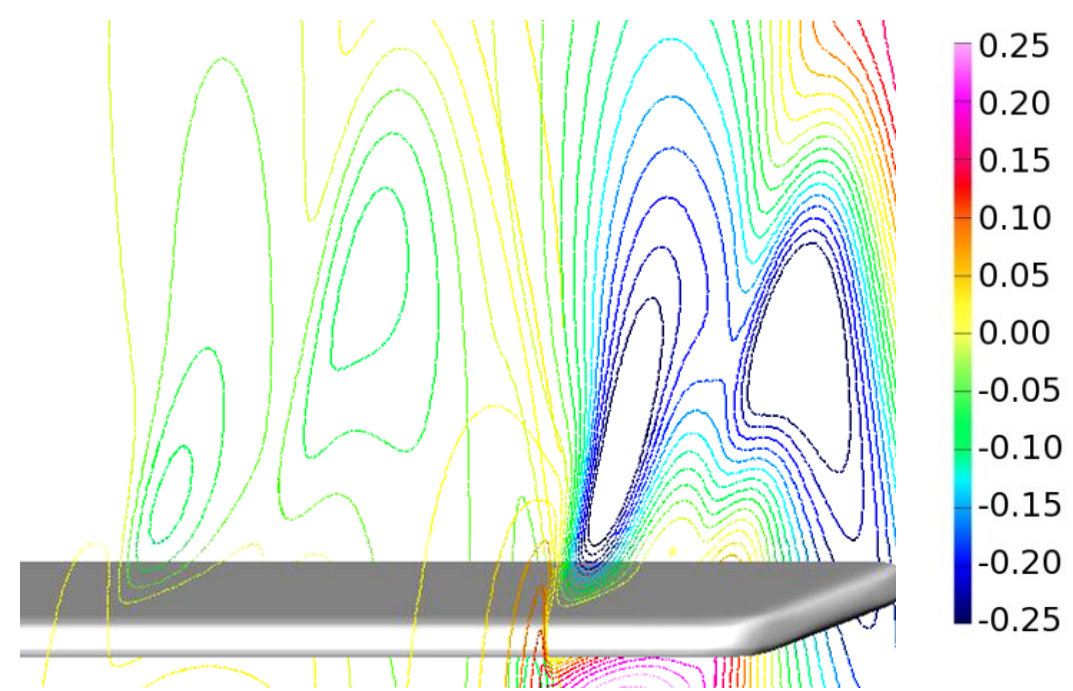

Figure 73. Spanwise velocity contours at $62.5 \%$ and $87.5 \%$ of span. The spanwise velocity is much stronger near the wingtips than near midspan, though still relatively weak compared to the freestream velocity.

\section{F. Tip Vortices and Wing Interaction}

Figure 74-Figure 76 shows the interaction between tip vortices from the forewing and hindwing by plotting the iso-surfaces of the vorticity about the $\mathrm{x}$-axis. The tandem wing tip vortices are presented alongside the tip vortices of the single wing for comparison. Iso-surface contours of the vorticies are shown at different time instants of the flapping cycle. 


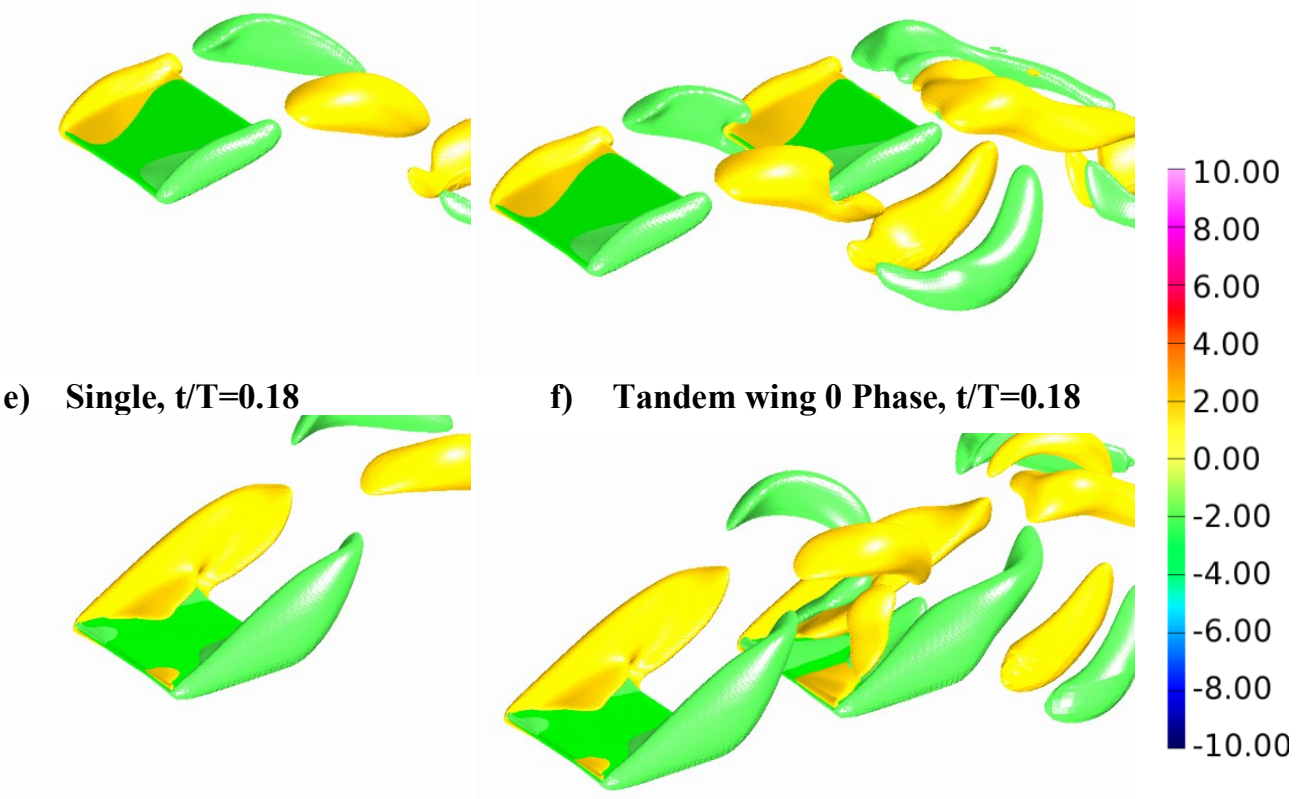

g) Single, $t / \mathbf{T}=\mathbf{0 . 4 5}$

h) Tandem wing 0 Phase, $t / T=0.45$

Figure 74. The tip vortex interaction between the fore and hind wings at $0^{\circ}$ phase lag. In this case the hindwing bisects tip vortices shed by the forewing on both the downstroke and upstroke.

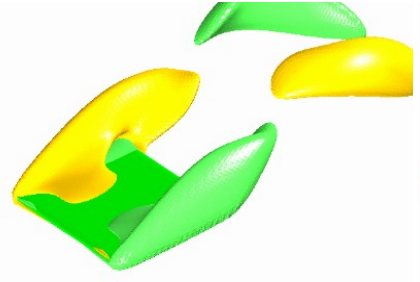

e) Single, $t / \mathbf{T}=\mathbf{0 . 3 6}$

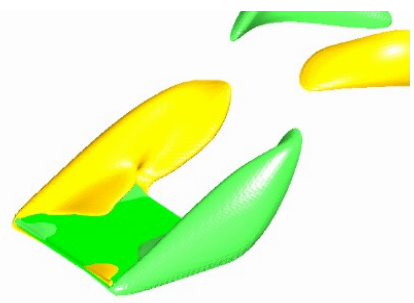

g) Single, $\mathbf{t} / \mathbf{T}=\mathbf{0 . 4 5}$

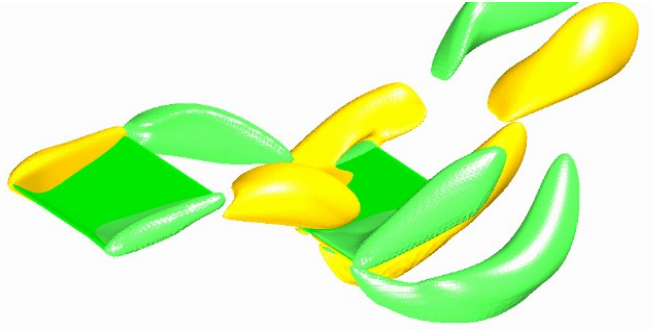

f) Tandem wing 90 Phase, $t / T=0.36$

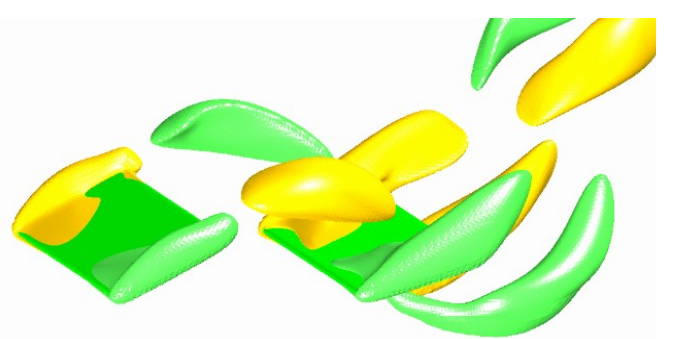

h) Tandem wing 90 Phase, $t / T=0.45$
10.00
8.00
6.00
4.00
2.00
0.00
$-2.00$
$-4.00$
$-6.00$
$-8.00$

$-10.00$

Figure 75. The tip vortex interaction between the fore and hind wings at $90^{\circ}$ phase lag. In this case there is no noticeable interaction between tip vortices shed by the forewing with the hindwing. 


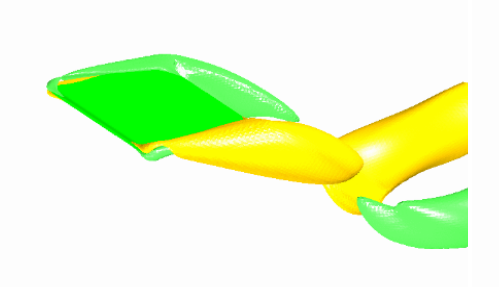

e) Single, $t / T=0.99$

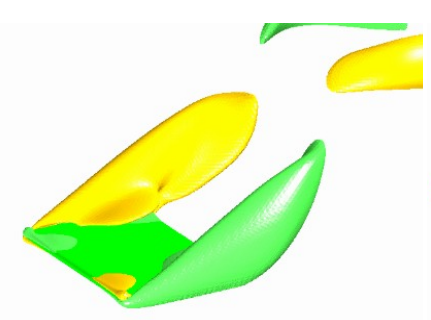

g) Single, $t / T=0.5$

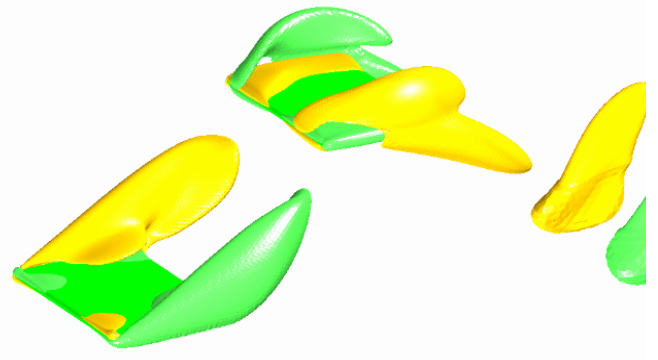

f) Tandem wing 180 Phase, $t / T=0.99$

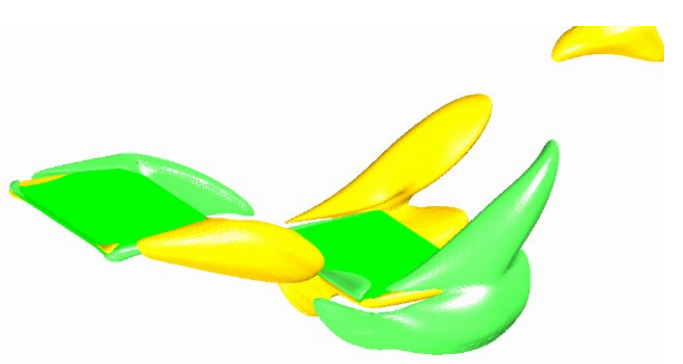

h) Tandem wing 180 Phase, $t / T=0.5$
10.00

8.00

6.00

4.00

2.00

0.00

$-2.00$

$-4.00$

$-6.00$

$-8.00$

$-10.00$

Figure 76. The tip vortex interaction between the fore and hind wings at $180^{\circ}$ phase lag. In this case the tip vortices shed by the forewing interact with the hindwing at the start of both the upstroke and downstroke.

For the most part, the tip vortex generation of the hindwing only shows minor variation with phase angle. The timing of the tip vortex formation and shedding is independent of phase angle. The hindwing always starts to form the tip vortex at the start of a stroke and sheds the tip vortex at the end of a stroke. There are, however; interactions that occur between the tip vortices shed by the forewing and those generated by the hindwing which vary with phase angle. At $0^{\circ}$ phase lag, on both the upstroke and downstroke, the hindwing bisects the tip vortices shed from the forewing, which interact with opposite signed tip vortices generated by the hindwing. The interaction on the downstroke is shown in Figure 74b. Despite this interaction, the tip vortices shed by the hindwing (Figure 74d) are slightly larger than those generated by a single wing. For the $90^{\circ}$ case, the tip vortices shed by the forewing pass both above and below the hindwing without any interaction (Figure 75b). Even without interaction, the tip vortices shed by 
the hindwing are slightly weaker than those of the single wing. Finally, the hindwing of the $180^{\circ}$ passes through the tip vortices of the forewing at the start of both the upstroke and downstroke which interact with the opposite signed tip vortices generated by the hindwing (Figure 76b, d). The tip vortices shed by the hindwing in this case are noticeably smaller than those of the single wing, possible due to this destructive interaction. The effect of these tip vortex interactions, however; is minimal as the tip vortices are confined to the very tip of the wing. 


\section{CONCLUSIONS}

Improving the design of micro air vehicles (MAVs) requires an in-depth understanding of flapping wing aerodynamics. While a large body of research exists regarding single flapping wing configurations, the study of tandem flapping wing configurations is lacking. In this dissertation, a tandem flapping wing configuration was studied at conditions similar to MAV flight. The effect of wing-wing vortex interactions on the force production and power consumption was analyzed, information that could help improve future MAV designs. Previous literature on the subject was covered in section I, while section II explained the computational method used in the study.

In section III, the aerodynamics of a tandem flapping wing configuration in forward flight was analyzed at a Reynolds number of 10,000. Three different phases between the fore and hind wings were considered: 0, 90, and 180 degrees, and the gap between the fore and hind wing was equal to one chord length. The analysis was performed at a Strouhal number of 0.3. Detailed comparisons were made with an isolated single flapping wing in terms of the lift, thrust, resultant force, and power coefficients, the propulsive, lift and resultant efficiencies and the vorticity contours.

The effect of phase angle was studied on the fore and hind wings, individually. Compared to a single wing, the forewing, regardless of phase angle, showed increased lift, thrust and resultant coefficients. The hindwing had a smaller lift coefficient at all three phase angles, but the $0^{\circ}$ hindwing showed a large increase in the thrust coefficient, 
while the $90^{\circ}$ and $180^{\circ}$ hindwings showed a large decrease. The propulsive efficiency of the forewing was unchanged from the value for a single wing, at all phase angles. At $0^{\circ}$ and $90^{\circ}$ phase lag, the forewing showed a noticeable decrease in the lift and resultant efficiencies, but at $180^{\circ}$ there was a slight increase. For the hindwing, at $0^{\circ}$ phase lag, there was a significant increase in the propulsive efficiency compared to a single wing, but a very large decrease in the lift and resultant efficiencies. The opposite was true of the $90^{\circ} / 180^{\circ}$ hindwings, which showed a large decrease in the propulsive efficiency, but significantly higher lift and resultant efficiencies. At $90^{\circ}$ and $180^{\circ}$, the hindwing requires only $36 \%$ and $43 \%$ of the power necessary to actuate a single wing. Given that all wings are undergoing the same flapping kinematics, this shows that the hindwing benefits from the presence of the forewing by extracting energy from the wake of the forewing at these two phase angles.

The effect of phase angle on the tandem configuration was also evaluated at a systems level and its performance compared to a single wing. The lift coefficient of the tandem configuration was lower than a single wing, but relatively constant at all three phase angles. At $0^{\circ}$ phase lag, the thrust coefficient was much higher than a single wing's, while at $90^{\circ} / 180^{\circ}$ it was much lower. The resulted in the $0^{\circ}$ phase case having the same resultant coefficient as a single wing, but with it inclined more forward, while the $90^{\circ} / 180^{\circ}$ cases had a $20 \%$ smaller resultant coefficient than a single wing. Flapping in $0^{\circ}$ phase resulted in the largest power coefficient, significantly larger than a single wing. The $90^{\circ}$ and $180^{\circ}$ phase configurations reduced the normalized power consumption to less than $50 \%$ of the $0^{\circ}$ phase case. The propulsive efficiency of the $0^{\circ}$ phase case was slightly higher than a single wing, but the lift and resultant efficiencies were significantly 
lower. Conversely, the $90^{\circ} / 180^{\circ}$ cases have slightly lower propulsive efficiencies than a single wing, but nearly the same lift and resultant efficiencies. Adjusting the phase angle allows the ability to switch between two different flight modes. When flapping at $0^{\circ}$ phase, the tandem configuration produces large aerodynamic forces, especially thrust, at high propulsive efficiency, but at the cost of lift efficiency. This would be suitable for high performance maneuvers. Switching to a $90^{\circ}$ or $180^{\circ}$ phase flapping cycle greatly reduces the power consumption at the cost of thrust production. Propulsive efficiency goes down slightly, but lift and resultant efficiency increase significantly. Since the net thrust is still greater than zero, but the power cost is much lower, this would be suitable for cruising flight. These results mirror the observations of natural dragonflies in flight.

Finally, the relationship between phase angle and wing-wing vortex interactions was studied. Adjusting the phase angle of the tandem configuration changed the nature of fore and hind wing interactions, affecting LEV formation and the resulting force generation. The $0^{\circ}$ phase case was characterized by constructive vortex interactions between the fore and hind wing. These interactions increased the size of the LEVs generated by the hindwing which resulted in increases in the peak lift and thrust production.

In section IV, the aerodynamics of a tandem flapping wing configuration in forward flight was analyzed at a Reynolds number of 5000. In this section the effect of the wing spacing between the fore and hind wings was analyzed. Four different spacings were considered, $0.1 \mathrm{c}, 0.25 \mathrm{c}, 0.5 \mathrm{c}$ and $1.0 \mathrm{c}$, at three different phase angles, 0,90 , and 180 degrees. The analysis was performed at a Strouhal number of 0.3. Detailed comparisons were made with an isolated single flapping wing in terms of the lift, thrust, resultant 
force, and power coefficients, the propulsive, lift and resultant efficiencies and the vorticity contours.

The $90^{\circ} / 180^{\circ}$ cases were characterized by destructive vortex interactions between the fore and hind wing. These interactions decreased the size of the LEVs generated by the hindwing as well as affected the timing of LEV formation. This resulted in decreased peak lift and thrust production. It also caused a phase shift in lift and thrust production, causing both cases to produce significant amounts of positive lift on the upstroke which reduced the power coefficient.

A numerical investigation had been conducted to understand the effects of phase lag and spacing on the vortex/wing and vortex/vortex interactions, as well as the force generation and efficiencies of a tandem wing configuration with an emphasis on the hindwing. The results from this current study suggest that the force production and efficiency of the hindwing are heavily influenced by its interaction with the wake of the forewing, and that the nature of this interaction can be controlled by adjusting both the phase angle between the fore and hind wings and their spacing. For the kinematics and flow conditions used in this paper, both the phase angle and spacing affect the specific timing that the hindwing passes through the vortex shed from the forewing.

The interaction between the shed vortex and the hindwing also influences the LEV generation of the hindwing. Changes in the phase angle or spacing affect the timing of this interaction which, in turn, affects the timing of the generation and shedding of the LEV on the hindwing. The interaction between the shed vortex and the LEV can be described as constructive or destructive. Nearly every case studied in this paper exhibits constructive vortex interaction, where the directions of rotation of the shed vortex and the 
LEV are the same. This interaction reinforces the LEV and results in larger and stronger LEV formation than the single wing with no interaction. Only one case studied here exhibits destructive vortex interaction and that is the $180^{\circ}$ case with $1.0 \mathrm{c}$ spacing. In this case, the directions of rotation of the shed vortex and LEV were opposite of each other. This dampens the LEV formation and quickens the shedding, resulting in the formation of a smaller LEV than the single wing with no interaction. Unlike previous studies, which typically show that peak resultant forces are generated at $0^{\circ}$ phase angle and peak power efficiency occurs out of phase, our study shows that at different spacings the peak force generation and peak efficiencies occur at different phase angles.

The timing of the LEV generation and shedding corresponds to the phase lag observed in the lift and thrust data associated with changes in the spacing and phase angle while the size of the LEV generated corresponds to the peak lift and thrust production. This suggests that changes in the phase angle and spacing can be use to control the force production and efficiency of the hindwing by controlling its LEV formation. Both the phase angle and spacing were observed to have similar effects on the force production, which corresponded to similarities in the LEV formation and shedding. Specifically, for the cases studied, an increase in the phase angle of $90^{\circ}$ was observed to have a similar effect as decreasing the spacing by $0.75 \mathrm{c}$. This specific correspondence is almost assuredly a function of the flight speed and flapping kinematics used in the study.

The 3D results exhibited the same constructive and destructive vortex interactions that were observed in the $2 \mathrm{D}$ case, as well as the same relationship between phase angle and force production. That is, when the wings flapped with $0^{\circ}$ phase lag, there was constructive vortex interaction between the fore and hindwings, increasing the size of the 
LEV generated by the hindwing and the resulting lift and thrust production. With $180^{\circ}$ of phase lag, the vortex interactions were destructive, which reduced the size of the LEV generated by the hindwing along with the associated lift and thrust production. The transient and time averaged forces of the three dimensional case, however, were noticeably lower than the two dimensional results, due to the finite aspect ratio of the wing. Furthermore, tip vortices partially suppressed LEV formation near the edge of the wing and weakened the LEV formation at midspan. Weaker vortex shedding from the forewing resulted in weaker vortex interaction between the fore and hind wings than that observed in the two dimensional case. Finally, while the 3D tandem wing exhibited tip vortex interactions between the fore and hind wings, the resulting interactions were insignificant as the tip vortices were confined to the tip of the wing. In conclusion, the two dimensional case does capture the salient interactions between the fore and hind wings, but it over predicts the strength of the vortex interactions between the fore and hind wings and, thus, the resulting lift and thrust generation.

Finally, there are several areas that could be expanded for future work related to this project. First, the tandem wing could be tested at more phase angles such as $5^{\circ}, 10^{\circ}$, $45^{\circ}$ as well as phase angles beyond $180^{\circ}$. It would also be worthwhile to attempt to model the phase relationship using a non dimensional parameter. Such a relationship would likely include the flight velocity, wing spacing and flapping frequency. Furthermore, the lift and thrust data could be fitted with a Fourier series in order to detect new trends. And finally, the code could be modified to run on parallel processors in order to greatly decrease the required runtime for a simulation. 


\section{REFERENCES}

[1] Grasmeyer, J.M. and Keennon, M.T., "Development of the Black Widow Micro Air Vehicle," AIAA Paper No. 2001-0127, 2001.

[2] Pornsin-Sirirak, T.N., Tai, Y-C., Ho, C-M., and Keennon, M., "Microbat: A PalmSized Electrically Powered Ornithoper," http://ho.seas.ucla.edu/publications/conference/2001/jpl10_2001.pdf.

[3] McMasters, J. H., and Hendersen, M. L., "Low Speed Single Element Airfoil Synthesis," Technical Soaring, Vol. 6, No. 2, 1980.

[4] Pines, Darryll J., Bohorquez, Felipe, "Challenges Facing Future Micro-Air-Vehicle Development," Journal of Aircraft, Vol. 43, No. 2, 2006.

[5] Platzer, Max F.,Jones, Kevin D., Young, John, Lai, Joseph C. S., "Flapping-Wing Aerodynamics: Progress and Challenges," AIAA Journal, Vol. 46, No. 9, 2008.

[6] Shyy, W., Lian, Y., Tang, J., Viieru, D., and Liu, H., "Aerodynamics of Low Reynolds Number Flyers," Cambridge University Press, 2008.

[7] Taylor, G. K., et al., "Flying and Swimming Animals Cruise at a Strouhal Number Tuned for High Power Efficiency," Nature, Vol. 425, pp. 707-711 (2003)

[8] Knoller, R., "Die Gesetze des Luftwiderstandes," Flug- und Motortechnik (Wien), Vol. 3, No. 21, 1909.

[9] Betz, A., "Ein Beitrag zur Erklaerung des Segelfluges," Zeitschrift fur Flugtechnik und Motorluftschiffahrt, Vol. 3, 1912.

[10] http://www.aa.nps.navy.mil/programs/aero/propulsion/

[11] Garrick, I. E., "Propulsion of a Flapping and Oscillating Airfoil," NACA, Rept. 567, 1936.

[12] Jones, K. D., et al., "Experimental and Computational Investigation of Flapping Wing Propulsion for Micro Air Vehicles," Progress in Astronautics and Aeronautics, Vol. 195, pp. 307-339 (2001) 
[13] Ramamurti, R. and Sandberg, W., "Simulation of Flow about Flapping Airfoils Using Finite Element Incompressible Flow Solver," AIAA Journal, Vol. 39, No. 2, pp. 253-260 (2001)

[14] Viieru, Dragos, Tang, Jian, Lian, Yongsheng, Liu, Hao, Shyy, Wei., "Flapping and Flexible Wing Aerodynamics of Low Reynolds Number Flight Vehicles," AIAA Paper 2006-503, Reno, NV, January 2006.

[15] Weis-Fogh, T., "Quick Estimate of Flight Fitness in Hovering Animals, Including Novel Mechanisms for Lift Production," Journal of Experimental Biology, Vol. 59, 1973.

[16] May, M. L., "Dragonfly flight: power requirements at high speed and acceleration," The Journal of Experimental Biology, Vol. 158, pp. 325-342 (1991)

[17] Reavis M.A. and Luttges M.W., "Aerodynamic forces produced by a dragonfly," AIAA Journal, No. 88-0330, pp. 1-13 (1988)

[18] Schmidt, W., "Der Wellpropeller, ein Neuer Antrieb fuer Wasser-, Land-, und Luftfahrzeuge," Zeitschrift fur Flugwissenschaften, Vol. 13, pp. 427-479 (1965)

[19] Bosch, H., Interfering Airfoils in Two-Dimensional Unsteady Incompressible Flow, CP-277, AGARD, Paper 7, Sept. 1977.

[20] Tuncer, I. H., and Platzer, M. F., "Thrust Generation Due to Airfoil Flapping," AIAA Journal, Vol. 34, No. 2, pp. 324-331 (1996)

[21] Alexander, D.E., "Unusual phase relationships between forewings and hindwings in flying dragonflies," Journal of Experimental Biology, Vol. 109, pp. 379-383 (1984)

[22] Ruppell, G., "Kinematic analysis of symmetrical flight maneuvers of Odonata," The Journal of Experimental Biology, Vol. 144, pp. 13-42 (1989)

[23] Azuma, A., and Watanabe, T., "Flight Performance of a Dragonfly," The Journal of Experimental Biology, Vol. 137, pp. 221-252 (1988)

[24] Thomas, Adrian, et al., "Dragonfly flight: free-flight and tethered flow visualizations reveal a diverse array of unsteady flight-generating mechanisms, controlled primarily via angle of attack," The Journal of Experimental Biology, Vol. 207, pp. 4299-4323 (2004)

[25] Lan, C. E., "The Unsteady Quasi-Vortex-Lattice Method with Application to Animal Propulsion," Journal of Fluid Mechanics, Vol. 93, No. 4, pp. 747-765 (1979) 
[26] Usherwood, J. R. and Lehmann, F.O., "Phasing of dragonfly wings can improve aerodynamic efficiency by removing swirl," J. R. Soc Interface, Vol. 5, No. 28, pp. 1303-1307 (2008)

[27] Maybury, W., and Lehmann, F.O., "The fluid dynamics of flight control by kinematic phase lag variation between two robotic insect wings," The Journal of Experimental Biology, Vol. 207, pp. 4707-4726 (2004)

[28] Yamamoto, M., and Isogai, K., "Measurement of Unsteady Fluid Dynamic Forces for a Mechanical Dragonfly Model,” AIAA Journal, Vol. 43, No. 12, pp. 2475-2480 (2005)

[29] Wang, Z. and Russell, D., "Effect of forewing and hindwing interactions on aerodynamic forces and power in hovering dragonfly flight," Physical Review Letters, Vol. 99, No. 14, pp. 1-4 (2007)

[30] Lan, S.L. and Sun, M., "Aerodynamic force and flow structures of two airfoils in flapping motions," Acta Mechanica Sinica., Vol. 17, No. 4, pp. 310-331 (2001)

[31] Sun, M. and Lan, S.L., "A computational study of the aerodynamic forces and power requirements of dragonfly (Aeschna juncea) hovering," The Journal of Experimental Biology Vol. 207, pp. 1887-1901 (2004)

[32] Isogai, K., et al., "Unsteady Three-Dimensional Viscous Flow Simulation of a Dragonfly Hovering," AIAA Journal, Vol. 42, No. 10, pp. 2053-2059 (2004)

[33] Warkentin, Jonathan and DeLaurier, James, "Experimental Aerodynamic Study of Tandem Flapping Membrane Wings," Journal of Aircraft, Vol. 44, No. 5, pp. 1653 1651 (2007)

[34] Saharon, D., and Luttges, M., “Three-Dimensional Flow Produced by a PitchingPlunging Model Dragonfly Wing," 25th AIAA aerospace sciences meeting, AIAA Paper 87-0121, (1987)

[35] Saharon, D., and Luttges, M., "Visualization of Unsteady Separated Flow Produced by Mechanically Driven Dragonfly Wing Kinematics Model,” 26th AIAA aerospace sciences meeting, AIAA Paper 88-0569, (1988)

[36] Saharon, D., and Luttges, M., "Dragonfly Unsteady Aerodynamic: The Role of the Wing Phase Relations in Controlling the Produced Flows," 27th AIAA aerospace sciences meeting, AIAA Paper 89-0832, (1989) 
[37] Akhtar, I., et al., "Hydrodynamics of a biologically inspired tandem flapping foil configuration," Theoretical and Computational Fluid Dynamics, Vol. 21, No. 3, pp. 155-170 (2007)

[38] Huang, Hua and Sun, Mao, "Dragonfly Forewing-Hindwing Interaction at Various Flight Speeds and Wing Phasings," AIAA Journal, Vol. 45, No. 2, pp. 508-511 (2007)

[39] Wang, J., and Sun, M., "A computational model of the aerodynamics and forewinghindwing interaction of a model dragonfly in forward flight," The Journal of Experimental Biology, Vol. 208, pp. 3785-3804 (2005)

[40] Broering, T., Lian, Y., and Henshaw, W., "Numerical Investigation of Energy Extraction in a Tandem Flapping Wing Configuration," Manuscript accepted for publication by AIAA Journal.

[41] Rival, D., Hass, G., and Tropea, C., "Recovery of Energy from Leading- and Trailing-Edge Vortices in Tandem-Airfoil Configurations," Journal of Aircraft, Vol. 48, No. 1, pp. 203-211 (2011)

[42] Lim, K.B., and Tay, W.B., "Numerical analysis of the s1020 airfoils in tandem under different flapping configurations," Acta Mech Sin, Vol. 26, pp. 191-207 (2010)

[43] Henshaw, W.D. and Petersson, N.A., "A Split-Step Scheme for the Incompressible Navier-Stokes Equations," Numerical Simulation of Incompressible Flows, World Scientific, River Edge, pp. 108-125 (2003)

[44] S. Balay, W. D. Gropp, L. C. Mcinnes, and B. F. Smith, The portable extensible toolkit for scientific computation, Tech. Rep.

http://www.mcs.anl.gov/petsc/petsc.html, Argonne National Laboratory (1999)

[45] Young, J., "Numerical Simulation of the Unsteady Aerodynamic Flapping Airfoils. Ph.D dissertation, School of Aerospace, Civil and Mechanical Engineering, The University of New South Wales/Australian Defence Force Academy (2005)

[46] Young, J., Lai, J., and Germain, C., "Simulation and Parameter Variation of Flapping-Wing Motion Based on Dragonfly Hovering," AIAA Journal, Vol. 46, No. 4, pp. 918-924 (2008) 
[47] Lian, Y., and Shyy, W., "Aerodynamics of Low Reynolds Number Plunging Airfoil Under Gusty Environment," 45th AIAA Aerospace Sciences Meeting and Exhibit, AIAA Paper 2007-71 (2007)

[48] Ol, M. (Ed.), “Unsteady Aerodynamics for Micro Air Vehicles," NATO RTO AVT149 Report (2010)

[49] Henshaw, W.D. and Schwendeman, D.W., "Moving Overlapping Grids with Adaptive Mesh Refinement for High-Speed Reactive and Non-reactive Flow," Journal of Computational Physics, Vol. 216, pp. $744-779$ (2006)

[50] Wakeling, J. M. and Ellington, C. P., "Dragonfly flight: II: Velocity, acceleration, and kinematics of flapping flight," The Journal of Experimental Biology, Vol. 200, pp. 557-582 (1997)

[51] McCroskey, W.J., et al., "Dynamic Stall Experiment on Oscillating Airfoil," AIAA Journal, Vol. 14, 1976, pp. 57-63.

[52] Visbal, M. and Shang, J., "Investigation of the Flow Structure around a Rapidly Pitching Airfoil," AIAA Journal, Vol. 27, No. 8, 1989, pp. 1044-1051.

[53] Liu, H. and Kawachi, K., "A Numerical Study of Insect Flight," Journal of Computational Physics, Vol. 146, No. 1, 1998, pp. 124-156.

[54] Wakeling, J. M. and Ellington, C. P., "Dragonfly flight: II: Velocity, acceleration, and kinematics of flapping flight," The Journal of Experimental Biology, Vol. 200, pp. 557-582 (1997)

[55] Azuma, A., et al., "Flight Mechanics of a Dragonfly," The Journal of Experimental Biology, Vol. 116, 1985, pp. 79-107.

[56] Jones, K. D., et al., "Bio-Inspired Design of Flapping-Wing Micro Air Vehicles," The Aeronautical Journal, Vol. 109, No. 1098, 2005, pp. 385-393.

[57] Birch, J. M., and Dickinson, M. H., "Spanwise Flow and the Attachment of the Leading-Edge Vortex on Insect Wings,” Nature (London), Vol. 412, 2001, pp. 729 733.

[58] Ellington, C. P., van den Berg, C., Willmott, A. P., and Thomas, A. L. R., "LeadingEdge Vortices in Insect Flight," Nature (London), Vol. 384, 1996, pp. 626-630. 


\section{CURRICULUM VITAE}

Name: Tim Broering

Address: 2019 Woodbourne Ave.

Louisville, KY, 40205

DOB: $10 / 31 / 1985$

Education

And Training: B.S. Mechanical Engineering

University of Louisville

August, 2008

M.Eng. Mechanical Engineering

University of Louisville

May, 2009

Awards: Hsing Chuang Award for Excellence in Graduate Study

Senior Year Academic Achievement Award for Mechanical Engineering

Grawemeyer Scholar

Dean's Scholar

University Honor's Program

Publications: Broering, T. and .Lian, Y., Numerical Investigation of Energy Extraction in a Tandem Flapping Wing Configuration. AIAA Journal, 2012. p. 22952308.

Broering, T. and Lian, Y., The effect of phase angle and wing spacing on tandem flapping wings. Acta Mechanica Sinica, 2012. p. 1557-1571. 